\title{
Intricate Identities \\ Cochlear Implant Users Negotiating Lives Between d/Deaf and Hearing Worlds
}

Hayley Bathard

A thesis submitted to Victoria University of Wellington in fulfilment of the requirements for the degree of Masters of Arts in Cultural Anthropology.

Victoria University of Wellington 
The cochlear implant $(\mathrm{Cl})$, a device that "provides hearing sensations for severely and profoundly deaf individuals" (NZ Audiological Society), initially emerged for public use in the 1980s, but was met with strong opposition from Deaf communities in many countries (Lane et al 1996, Edwards 2005). However, since the beginning of the $21^{\text {st }}$ century, hostility towards implants has lessened and they are increasingly accepted as an option in a range of possibilities for deaf children and adults. Despite increasing numbers of the Deaf community considering implants themselves, however, the continuing task of the Deaf community is to counter the conception of implants as 'miracle cures' for deafness (Lane et al 1996, Edwards 2005). Furthermore, the Deaf community needs to communicate to parents of deaf children that those with implants may still be perceived as $d /$ Deaf, by both the community and themselves (Christiansen and Leigh 2002).

This thesis explores the identities of a small group of cochlear implant users in New Zealand, and examines their involvement in both $d /$ Deaf and hearing worlds. The narratives of my participants demonstrate some of the everyday difficulties that $d /$ Deaf individuals, and their families, encounter in medical and health-care settings, along with educational and workplace settings. I draw on participants' narratives that explain their relationship with both medicalised and cultural models of deafness, and with Deaf culture, decisions about implants, and perceptions of the effects and limits of cochlear implants. I argue that the identities of $\mathrm{Cl}$ users in modern New Zealand society are influenced by a multiplicity of factors, including medicalised understandings of deafness, familial pressures, the embodied experiences of $\mathrm{Cl}$ technology, and personal identity trajectories. Given that these individuals are navigating these multiple threads in the formation of their identities, I argue that, at this stage in their lives, the $\mathrm{Cl}$ users in this study occupy a liminal position in regards to d/Deaf and hearing worlds. Furthermore, I posit that the medical model of deafness needs to be tempered with social and cultural views of both deafness and $\mathrm{Cls}$, and that the voices of $\mathrm{Cl}$ users themselves should be prominent in such debates. 


\section{ACKNOWLEDGEMENTS}

Firstly, acknowledgements must go to my participants, to whom I am so grateful for their time, thoughts, and invaluable contributions. I continue to be blown away by their insightful reflexivity and their openness. I hope that this thesis honours them and honestly represents their experiences.

I sincerely thank my primary supervisor, Catherine Trundle, for her constant support and guidance. I thank her for always pushing me to do my best work, and for her invaluable suggestions and input into my research. This thesis would not be quite the same without her recommendations and knowledge. Huge thanks also to my secondary supervisor, Rachel McKee. I thank her for her invaluable reading suggestions, discussions, useful inputs, and I am indebted to her extensive knowledge of the d/Deaf world. Lastly, I must also thank both Catherine and Rachel for their abilities to, without exception, make me feel more relaxed and confident in my work after each and every meeting. I could not have asked for better supervisors.

Thanks must also go to my proof-readers - my mum, who has always been there to help. And to Jared, for his dedication in the last hour. I also must thank my dad for his willingness to print and bind this thesis on the last day, despite it being an unreasonable request.

To Sam, for your never ending support, love and encouragement, and for your belief in me. This thesis, in all honesty, could not have happened without you - thank you for your social, emotional, and financial support, for enduring the emotional rollercoaster, and for making sure I was fed over the last weeks. For putting up with me unconditionally, I cannot thank you enough.

Lastly, to my parents, I would not be where I am today if it were not for you. Thank you for always being my biggest supporters and encouragers, no matter what my decision or direction. I must thank you especially for the numerous ways you have backed me over the last five years throughout my university education - it wouldn't have been possible without you. I am forever grateful for your belief in me, for always knowing what to say, and for your love. 


\section{TABLE OF CONTENTS}

ACKNOWLECT

CHAPTER TWO: THEORISING DEAF EXPERIENCES, BODIES AND SUBJECTIVITIES............................19

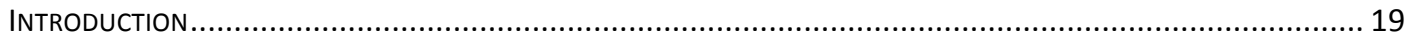

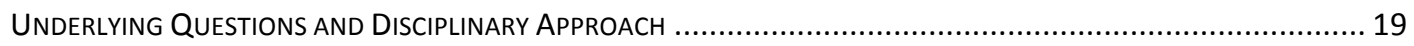

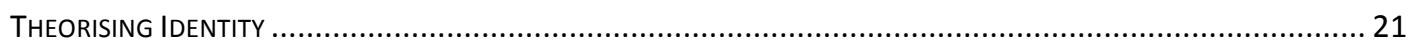

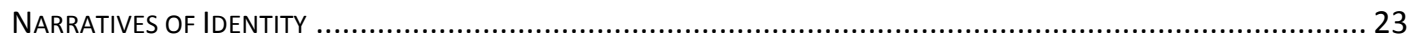

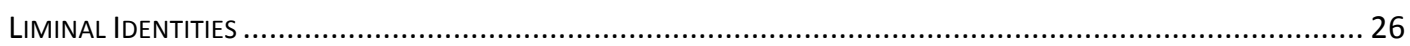

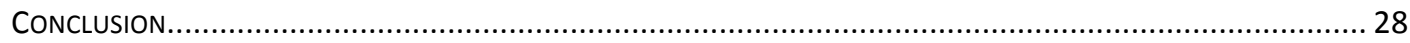

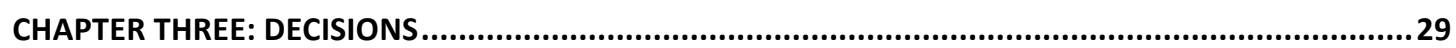

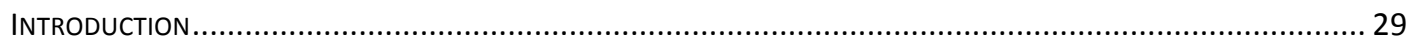

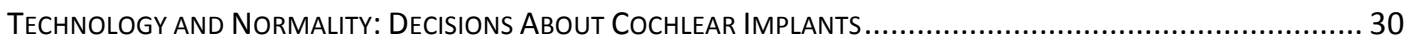

'The Limits of my Language MeAn the Limits of My WoRLD': The IMPORTANCE Of (SPOKEN) LANGUage ........ 36

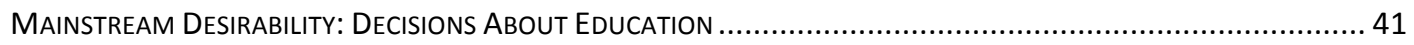

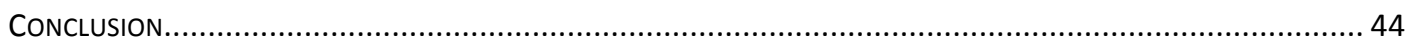

CHAPTER FOUR: NEGOTIATING LIFE WITH A COCHLEAR IMPLANT - THE EMBODIMENT OF

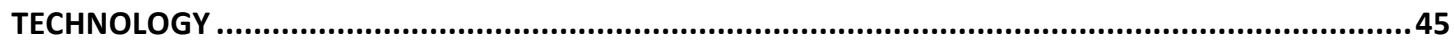

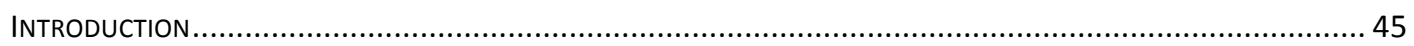

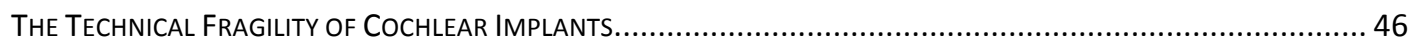

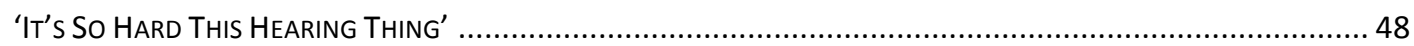

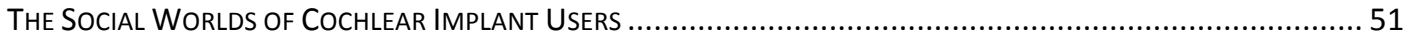


(MIS)UNDERSTANDINGS OF COCHLEAR IMPLANTS AND DeAfNeSS IN MAINSTREAM SOCIETY ............................. 54

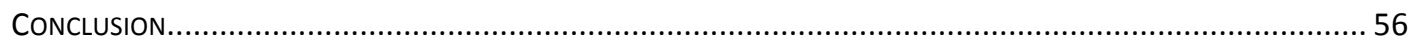

CHAPTER FIVE: NARRATIVES OF “CHOICE”: PROBLEMATISING THE IDEAL ....................................57

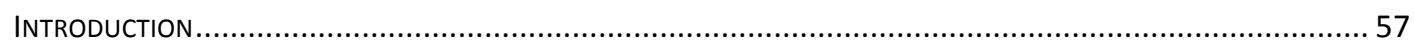

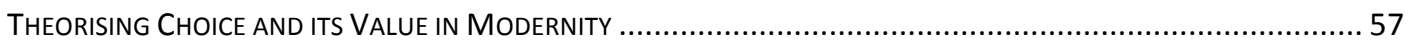

‘Give Her a Choice, Give Her a Chance'? Exploring Notions of “Choice” Through Cochlear Implants ... 60

CONCLUSION. 68

CHAPTER SIX: GROWING INTO d/DEAF IDENTITIES - ADOLESCENT IDENTITY WORK AND

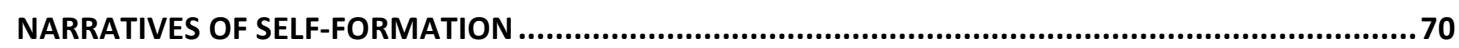

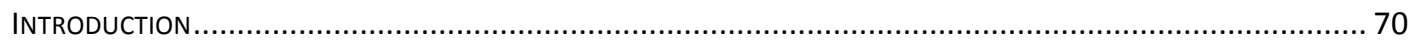

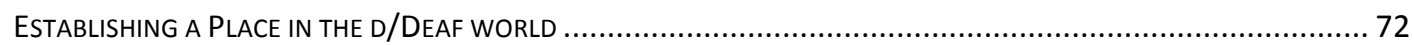

IDENTITY WORK AND THE ForMATION OF AN AUthentIC SENSE OF SELF............................................... 73

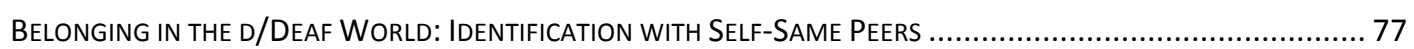

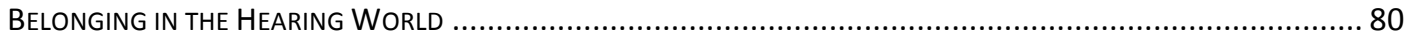

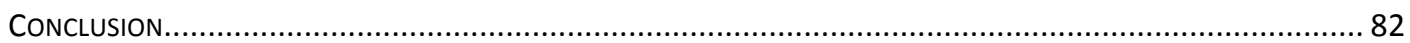

CHAPTER SEVEN: THE BEST OF BOTH WORLDS? NAVIGATING LIVES BETWEEN DEAF AND HEARING

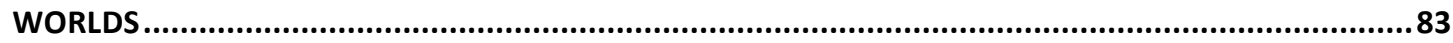

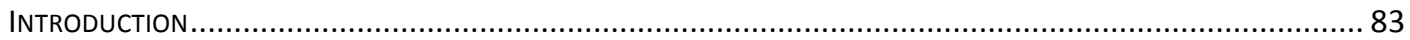

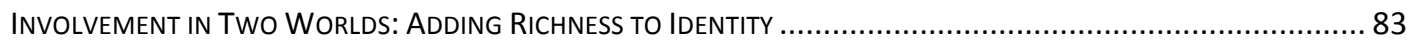

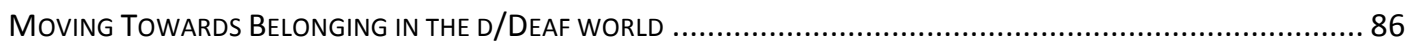

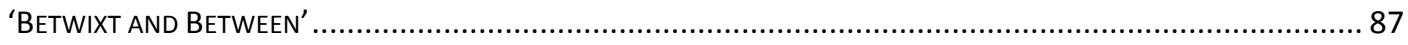

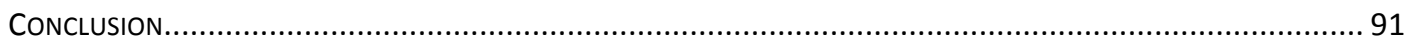

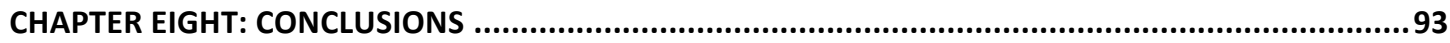

Recommendations From Participants: Good Practice for Cochlear IMPLANTS AND THEIR USERS ............. 95

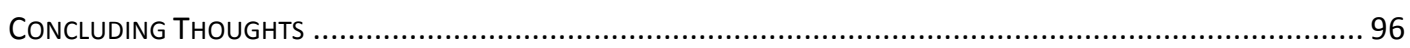

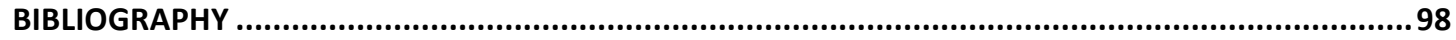




\section{CHAPTER ONE: INTRODUCTIONS}

I met Hannah at university one evening for our interview after she had completed a day of a Deaf Studies course. She was 22 years old and had been born deaf, and received her cochlear implant $(\mathrm{Cl})$ when she was five. She spoke in-depth about her thoughts on cochlear implants, noting, "Some deaf people might object to this, and I respect that, but I think implanting young children really young, do it so it's less traumatic for them - the operation and everything." However, Hannah was adamant that the process should not stop after implantation, and that $\mathrm{Cl}$ users need support after their surgery.

Do not just implant them and go, 'they'll be fine.' [...] Once you've done the operation, they still need support - they need speech therapy, they need interpreters, and everything. But one strong thing I have is once they're implanted, they [don't just] want [to], they need to learn sign language. [T]here's no argument, end of conversation [...].

She then turned the discussion to the way in which she believes $\mathrm{Cl}$ users should have the freedom to use the technology as they see fit, as they grow older.

[O]nce they have the implant, they have the choice, [...] 'I don't want it anymore,' or 'I want it.' Because if you decide not to implant them, by the time they're sixteen or seventeen, they go, 'I wanna hear,' it's too late, you know, so I do agree with the idea of implanting them quite young, [...]. I mean, it's sad because it's great to be deaf, I'm proud to be deaf [...]. And once they get older, they get a choice [...], that's what I strongly believe in - get them implanted when they're young, but when they're older don't force them to wear the implant if they don't want to. If they go, 'no I want to be deaf,' respect that. If they're [...] sixteen, seventeen, twenty, whatever, they go, 'I don't want to wear it anymore,' they're old enough! You know, they can drive, they can drink, they can do all of that if they don't want to hear, respect that, don't force them, [...] let them have the choice. 
I began this research project aiming to answer the following question: what are the effects of cochlear implants (Cls) on the identities of d/Deaf individuals in New Zealand? However, over the course of interviews with early adult cochlear implant users, their parents, and a teacher of the deaf, I came to realise that this project was about more than just the effects of technology on selfhood. Medical technologies are, after all, not separate from the social arenas and the cultural ideologies that produce them and within which they are used. I came to see that it was social experiences and expectations within both $d /$ Deaf and hearing worlds, kinship networks, medicalised ideals about the body, and cultural norms regarding the individual self, all mediated by the embodied experience of this technology, that shaped the identities of $\mathrm{Cl}$ users. I found that for participants to explain the experience of having a cochlear implant, it involved, centrally, talking about the complexity of entering and living within and between hearing and d/Deaf 'worlds.' Thus, this thesis seeks to explore how these individuals have forged their identities over time and through the multiple cultural threads and social influences that exist within $d /$ Deaf and hearing spheres.

This study is based on the data gained from in-depth interviews with ten participants, and seeks to proffer insights into the lived experiences of cochlear implant users, and their families, in New Zealand. I draw from and contribute to Deaf literature, disability studies, and medical anthropology. Whilst I am cautious of the use of the term 'disability' and the different meanings attached to it (especially in relation to deafness), I hope that this study can contribute to the relatively limited study of disability within anthropology, ${ }^{1}$ through an ethnographic exploration of $d /$ Deafness and cochlear implants, which treats deafness as part of the tapestry of human diversity. Deafness and $d /$ Deaf individuals have long been subjected to processes of normalisation (Bauman and Murray 2010) and medicalisation (Conrad 1992, 2005), in which attempts have been made, often in the form of medical 'treatments,' to alter deaf bodies so they fit into ideas of the 'norm' and into hearing society. These attempts are representative of a pathological view of deafness, "which focuses on how deaf people are different from hearing people and which generally perceives these differences negatively" (Baker and Cokely 1980: 54). Despite such efforts towards

\footnotetext{
1 "Anthropology's genuine fascination with otherness and the thickness of the ethnographic stance should be a boon to international disability studies. However, this promise is late in coming.... [And] compared to the plethora of work on illness and healing, medical anthropological research on disability has been minimal" (Kasnitz and Shuttleworth 2001: 3,5, in Rapp and Ginsburg 2012: 173).
} 
normalisation, a cultural view of deafness has emerged from within Deaf cultures globally. This view treats Deaf people as a "linguistic and cultural minority with distinctive mores, attitudes, and values and a distinctive physical constitution" (Lane 2002: 367-8). Hence, the use of a signed language is a defining feature of Deaf cultural minorities.

The cochlear implant, a device that "provides hearing sensations for severely and profoundly deaf individuals" (NZ Audiological Society), initially emerged for public use in the 1980s, but was met with strong opposition from Deaf communities in many countries (Lane et al 1996, Edwards 2005). For these communities, cochlear implants represented a new form of medical intervention aimed at 'curing' deafness (Lane et al 1996), and were accordingly perceived as a further threat to deaf bodies and Deaf culture. Whilst opinions about Cls have tempered over time (Padden and Humphries 2005, Christiansen and Leigh 2011), cochlear implants in young deaf children remain a concern within some sectors of the Deaf community. They represent the continuation of "dominant ideologies of normalcy that pigeonhole the meanings of deaf to medical constructions" (Bauman and Murray 2010: 211). Despite increasing numbers of the Deaf community considering implants themselves, the continuing task of the Deaf community is to counter the conception of implants as 'miracle cures' for deafness (Lane et al 1996, Edwards 2005). Furthermore, they need to communicate to parents of deaf children that those with implants are still perceived as d/Deaf (Christiansen and Leigh 2002).

This thesis explores the identities of a small group of cochlear implant users in New Zealand, and examines their involvement in both $d /$ Deaf and hearing worlds. The narratives of my participants demonstrate some of the everyday difficulties that $d /$ Deaf individuals, and their families, encounter in medical and health-care settings, along with educational and workplace settings. I draw on participants' narratives that explain their relationship with both medicalised and cultural models of deafness, and with Deaf culture, decisions about implants, and perceptions of the effects and limits of cochlear implants. I argue that the identities of $\mathrm{Cl}$ users in modern New Zealand society are influenced by a multiplicity of factors, including medicalised understandings of deafness, familial pressures, the embodied experiences of $\mathrm{Cl}$ technology, and personal identity trajectories. Given that these individuals are navigating these multiple threads in the formation of their identities, I argue that, at this 
stage in their lives, the $\mathrm{Cl}$ users in this study occupy a liminal position in regards to $\mathrm{d} /$ Deaf and hearing worlds. Furthermore, I posit that the medical model of deafness needs to be tempered with social and cultural views of both deafness and $\mathrm{Cls}$, and that the voices of $\mathrm{Cl}$ users themselves should be prominent in such debates.

\section{The Importance of Words: The Difference Between deaf and Deaf}

The words used to denote deafness reflect the various connotations associated with pathological and cultural views of deafness, where different spellings of 'deaf' can carry different meanings. Thus, despite common discussions amongst d/Deaf people, within Deaf culture and Deaf studies, and more widely within academia, about what $d /$ Deaf people do and do not want to be called, it is important here to draw a distinction between 'deaf' (with a lower case ' $d$ ') and 'Deaf' (with a capitalised ' $D$ '). Deaf Aotearoa New Zealand ${ }^{2}$ succinctly delineates between Deaf and deaf, explaining that the word Deaf "denotes a unique community. The use of sign language as one's first language is the principle characteristic of people who identify with this community" ${ }^{3}$ (DANZ 2012). Within the Deaf community, "Deaf people see being Deaf as a difference, not a disability. Being Deaf is a way of life" ${ }^{4}$ (DANZ 2012). On the other hand, deaf "refers to hearing loss - e.g. "he is deaf". The majority of people with hearing loss do not use sign language, as they generally become deaf late in life or are born with mild hearing loss" ${ }^{5}$ (DANZ 2012). Therefore, as Carol Padden asserts, Deaf is often used "when referring to cultural aspects," whilst deaf tends to refer to "non-cultural aspects such as the audiological condition of deafness" (in Skelton and Valentine 2003: 455).

Throughout this thesis, I will attempt to use the terms deaf and Deaf consistently, especially if it is clear which term is appropriate and intended. However, as Skelton and Valentine

\footnotetext{
${ }^{2}$ Deaf Aotearoa New Zealand (DANZ) is an organisation which provides resources and information for d/Deaf New Zealanders. It focuses on "promoting Awareness of, Access to and Advancement of New Zealand Sign Language - to help strengthen the rights of Deaf people and give them the confidence to be an active part of society" (DANZ 2012 - http://www.deaf.org.nz/about-us).

${ }^{3}$ http://www.deaf.co.nz/yk-files/4f61f39fc49013604518cc8d8914669d/Deaf+Community.pdf ${ }^{4}$ Ibid

${ }^{5}$ Ibid
} 
assert, " $[\mathrm{t}]$ he boundary between Deaf and deaf identities, meanings and construction can be fluid. At different times people may identify as Deaf or as deaf. In different spaces people may be ascribed an identity as Deaf or as deaf" (2003: 455-6). Thus, Skelton and Valentine (2003; Valentine and Skelton 2007), use D/deaf to reflect the fluidity and complexity of deaf identities. On using these terms together, Brueggemann states that the "twinning of $d /$ Deaf is perhaps safer that way, since often, when one is pressed, it is hard to determine at any one moment in a text whether the Big D cultural/linguistic arena is where we are or whether we are just in the small $d$ audiological/medical space. And what if we are in both places at the same time?" (Brueggemann 2009: 14).

Thus, throughout this thesis, drawing on Brueggemann, and Skelton and Valentine, and following on from Bathard (2012), I will frequently use d/Deaf to both deal with the complexity of labelling deafness and deaf individuals, as well as to acknowledge the fluidity, and possible hybridity, of identities. I will note here, however, that I tend to use 'd/Deaf' to refer to individuals, and 'Deaf' to denote collective Deaf social groups and spaces. While I do not propose a new solution for the labelling of deafness, I hope that this exploration of identities may help to further Brueggemann's ambition of examining "all the rhetorical situations that arise from the $d /$ Deaf distinctions, that bring the distinctions to bear, and that, most important, keep shifting them like an identity kaleidoscope in our own hands" (Brueggemann 2009: 15).

\section{THE POLITICS OF DEAFNESS}

Linguistic debates over the terms 'deaf' and 'Deaf' reflect broader discourses surrounding Deaf identity politics. These terms align with medical and social/cultural models of deafness, which draw, to an extent, on the medical and social models of disability (see Shuttleworth and Kasnitz 2004; Shakespeare 2005, 2006; Thomas 2007; Gray 2009; Landsman 2009; Oliver 2009). According to Senghas and Monaghan,

The medical model of deafness is one based on deficit theory and holds that deafness is the pathological absence of hearing and that such a hearing-impaired individual is therefore disabled because of faulty 
hearing. This perspective is sometimes called the medical model because medical procedures (such as cochlear implants) are characteristic of responses made by (hearing) parents of deaf children and often involve extensive intervention by medical professionals (2002: 78)

In contrast to the medical model, the cultural/sociocultural model of $d /$ Deafness identifies deafness "as one range within the larger spectrum of human variations," and "assumes that deafness allows for an alternate constellation of very human adaptations, among the most central being sign languages" (Senghas and Monaghan 2002: 78).

Given that over 90 per cent of deaf children are born to hearing parents (Dolnick 1993; Mitchell and Karchmer 2004), who are most likely to have little or no prior knowledge of deafness or Deaf culture, and who thus are inclined to be guided by professionals who generally adhere to the medical model of deafness, it is this model that tends to permeate wider society. As Lane et al state, hearing parents of deaf children "may be driven to begin a process of professionally guided identity development for their child that might appropriately be called 'the making of a hearing-impaired person.' The process begins with professional people, perhaps unwittingly, reinforcing the hearing parents' and hearing society's deficit model of their child" (1996: 30-1). Consequently, medical and audiological discussions are prevalent after diagnoses of deafness, with in-depth knowledge of Deaf culture, or the lived experiences of d/Deaf people, often being left out (Lane et al 1996). Furthermore, given the prevalence of the medical model of deafness, medical and educational interventions become central focuses of both professionals and parents.

Whilst intervention from the hearing world in the lives of $d /$ Deaf individuals has manifested in various ways throughout history, medical intervention is perhaps one of the most invasive. According to Lane et al,

The power of the hearing world vis-à-vis the DEAF-WORLD is nowhere more frightening than in the application of medical science to Deaf people. Medical interventions aimed at the cure of deafness have a long history. [...] Today, as the DEAF-WORLD sees it, this torch has been passed to those who would "cure" Deaf children by implanting electronic devices 
in their skulls and those who would prevent their birth in the first place by genetic testing and counselling and, when feasible one day, genetic engineering (1996: 371)

In this sense, ideals of normality are a powerful influence in the lives of $d /$ Deaf people, and still continue to play a central part in the 'treatment' of deafness within the medical world. However, despite this consistent strive towards normalcy and ongoing attempts to 'fix' or 'cure' deafness, Deaf people have been "remarkably consistent in denying that theirs is a medical condition in need of a cure" (Edwards 2005: 894). In contributing to these debates, I examine how $\mathrm{Cl}$ users' experiences with this 'normalising' technology actually shape their orientation to Deaf or hearing cultural worlds, arguing that their identities are much more complex and multi-layered than the polarities emphasised in these models of disability versus diversity, or medical normalisation versus Deaf culture.

\section{Differentiating Between Two Worlds: The Social Spaces of d/Deaf AND HEARING WORLDS}

Throughout this thesis, I refer to both the 'd/Deaf world' and the 'hearing world,' and use these terms primarily because my participants did so, in reference to their involvement in both deaf and hearing social and cultural arenas. Because it is not immediately obvious what the 'hearing world' in particular consists of, and because the word 'world' is potentially amorphous, I draw on Irene Leigh's (2009) work to explain these dichotomous terms. Leigh states that, for many deaf people, the phrase 'hearing world" "is taken to mean an auditory environment consisting of culturally sanctioned ways of communicating through spoken language, related gestures and facial expressions, attention-getting techniques, and vocal qualifiers, such as tone of voice" (Leigh 2009: 26-7). This hearing world "is most typically the environment of mainstream culture," and "[s]uch environments are contrasted with Deaf cultural ways of being in which people respond through visual means, including body language, facial expressions, signs or mouthed words, tapping one's shoulder for attention, and so on" (Leigh 2009: 27). Furthermore, according to Lane et al, Deaf people in the U.S. use the sign 'deaf-world' to refer to "relationships among themselves, to the social network they have set up, and not to any notion of geographical location" (1996: 5). 
For the most part, in the context of this research, I refer to the d/Deaf world in regards to my participants' involvement in Deaf groups and social spheres. This world was often encountered in the teenage years of these individuals, and tends to involve interaction with other $d$ /Deaf people, the use (and acquisition) of New Zealand Sign Language (NZSL), attendance at Deaf cultural events, and environments geared towards d/Deaf people (such as schools, clubs and deaf sporting events). The hearing world that my participants refer to indexes wider society, which, for the most part, adheres to hearing norms. This world incorporates family life (as all of the $\mathrm{Cl}$ users in this study were born to hearing parents), socialisation with hearing peers, as well as education and work in mainstream (hearing) settings.

It is important, however, to note the limitations of these terms and concepts, especially in that they strongly demarcate between these two 'worlds' or experiences in these different spheres, when in fact they overlap at times and in places, which I will explore throughout this research. Furthermore, these 'worlds' are not necessarily physical spaces in which my participants are situated, but rather often refer to social interactions and cultural experiences. Because the hearing world denotes mainstream society and everyday experience, my participants are always physically in the hearing world, and may make forays into the $d /$ Deaf social world.

\section{COCHLEAR IMPLANTS}

A "cochlear implant is an implanted electronic hearing device, designed to produce useful hearing sensations to a person with severe to profound nerve deafness by electrically stimulating nerves inside the inner ear" (FDA 2010). These sensations, or signals, "are interpreted by the brain and recognised as 'sound'" (Cochlear Implants in New Zealand). A cochlear implant provides access to sound for people who get little or no benefit from hearing aids (NFD), and consists of both internal and external parts. The external parts of a $\mathrm{Cl}$ "consist of a microphone, a speech processor and a transmitter coil" (Cochlear Implants in New Zealand). The microphone picks up sounds from around the user and passes the signals 
to the speech processor, which converts these into electrical signals, which are then sent via radio waves from the transmitter to the internal receiver (Cochlear Implants in New Zealand). The internal parts of the $\mathrm{Cl}$ are implanted in surgery, and are composed of a receiver with a magnet and a line of electrodes that is inserted into the cochlea (Cochlear Implants in New Zealand). Once the internal receiver picks up the signals, it sends these to the electrodes, which "directly stimulate the damaged hair cells of the cochlea, which sends the electrical signals along the nerve to the brain" (Cochlear Implants in New Zealand).

The New Zealand Audiological Society website lists a number of the benefits of cochlear implants, including, "improvement in the detection of environmental sounds," "increased ability to recognise speech with lip-reading," "an improved ability to understand speech using hearing alone," and "[i]mprovement in voice monitoring and speech production" (NZ Audiological Society 2011). However, the website also lists a number of the factors that influence the degree to which an individual who receives an implant will benefit from the technology. These include "[a]ge of onset of deafness; [p]revious experience of hearing; [d]uration of profound deafness," and "on-going habilitation" (NZ Audiological Society 2011). Furthermore, the website states that the "commitment and involvement of the individual/parents is vital" (NZ Audiological Society 2011). In this respect, whilst a Cl provides deaf individuals with the opportunity to hear some sounds, a considerable level of personal commitment and effort is required to learn to listen and make sense of the sounds.

The first cochlear implant, which stemmed from a long interest in schemes to 'restore' hearing to deaf individuals, was approved by the American FDA (Food and Drug Administration) for implantation in deaf adults in November 1984 (Blume 2010). Throughout the 1980s, implants were gradually accepted in medical and industrial circles, and, as clinical data was built up, "clinicians became enthusiastic, manufacturing corporations interested, regulatory bodies convinced, and health insurers willing to reimburse implantation" (Blume 2010: 67). By the end of the 1980s, ear surgeons were beginning to agree that the implant could also be of benefit to deaf children, and, in 1990, the FDA approved use of one brand of implant in children aged two to seventeen years (Blume 2010). Thus, over the course of five decades, scientific and professional opposition to implants have been overcome, numbers eligible for implants have been extended, and the device has become a commercial success 
(Blume 2010). As of December 2010, the Food and Drug Administration estimated that, around the world, 219,000 people had received a cochlear implant (Christiansen and Leigh 2011).

However, from the very introduction of cochlear implants, there has been opposition from Deaf communities across the world. This opposition has manifest in calls that $\mathrm{Cls}$ are yet another eugenic tool aimed to eliminate deafness (see Lane et al 1996, Edwards 2005), that it is unethical to implant children (see Christiansen and Leigh 2004, Hyde and Power 2006, Mitchiner and Sass-Lehrer 2011), with the most radical opponents of cochlear implants referring to the technology as a type of "cultural genocide" (Edwards 2005, Taylor 2011). In particular, there are widespread fears that Cls will profoundly affect signed languages. On this, Lane et al assert that cochlear implants signalled the reappearance of the oralist philosophy in a new guise - "one backed by all the authority of the modern medical establishment" (1996: 372). Therefore, for Deaf people and Deaf cultures the world over, cochlear implants have represented a threat to their culture, their languages and their Deaf pride. Furthermore, they represent a further attempt to 'normalise' deaf bodies when, for them, medical treatment is not necessarily welcome and does not mean progress; "[s]ince deafness is not a deprivation [...], talk of cures and breakthroughs and technological wizardry is both inappropriate and offensive" (Dolnick 1993: 41).

Since the beginning of the $21^{\text {st }}$ century, however, there has been a tempering of opposition to cochlear implants (Christiansen and Leigh 2002, Padden and Humphries 2005, Mitchiner and Sass-Lehrer 2011). Increasingly, they are accepted as an option in a range of possibilities for deaf children and adults. For example, in the United States, the National Association of the Deaf (NAD) "now formally supports cochlear implantation as one of a "multitude of options" that parents are encouraged to consider for their deaf child" (Christiansen and Leigh 2011: 39). It is worth noting, however, that there continues to be concern and controversy over the implantation of young children and the consequences of this (Mitchiner and Sass-Lehrer 2011). Furthermore, the growing acceptance of $\mathrm{Cls}$ has developed alongside the realisation that a cochlear implant does not make someone physically or psychologically hearing. In this respect, an implant does not take away or rid someone of their deafness. Instead, it is a tool through which they have the potential to 
access sound. Thus, Deaf communities have recognised that individuals with cochlear implants can still be affiliated with their communities, learn sign language and embrace Deaf culture.

In a New Zealand context, Deaf Aotearoa New Zealand (DANZ) does not have an explicit standpoint on cochlear implants, but it clearly adheres to a cultural view of Deafness (DANZ). The organisation focuses on the inclusion of all Deaf New Zealanders in society, and promotes awareness of, access to, and advancement of New Zealand Sign Language (DANZ) ${ }^{6}$. In what is perhaps an indication of the changing nature of Deaf communities and a reflection of changing attitudes towards cochlear implants in New Zealand Deaf communities, the current President of DANZ, Robert Hewison, has a cochlear implant (DANZ) ${ }^{7}$. Such a softening of attitudes towards cochlear implants in $d /$ Deaf communities in New Zealand is also reflected in the study by Fitzgerald et al (2013), with one participant in this study noting that $d /$ Deaf people no longer consider $\mathrm{Cls}$ "to be a judgement on $\mathrm{D} /$ deaf people as though they [are] somehow in need of fixing" (Fitzgerald et al 2013: 138). However, this participant "held no doubt that their widespread uptake is likely to be cultural genocide as they have the effect of decreasing the pool of people who are highly proficient in NZSL" (Fitzgerald et al 2013). In Snapp's (2012) study on hearing parents of children with cochlear implants, the parents placed emphasis on speech and the ability to hear. Hence, these parents enforced the "absolute normalcy of hearing as a fact and value" (Best et al 2013: 114-115), and did not see their choices on Cls for their children as 'decisions' (Snapp 2012). In this sense, these parents "assumed that the best thing for their child was that the child should be given access to normal hearing. The major issue parents emphasized was coming to terms with the 'fact' that their child was not 'normal' or 'perfect'" (Snapp 2012: 7). Furthermore, the study by Best et al (2013) draws on the concerns of two deaf parents about the referral system in New Zealand after newborn babies are found to be deaf. These parents were critical of the options given to parents because they "included only referrals to audiologists and information about hearing aids: nothing about Sign language" (Best et al 2013: 119). These two examples demonstrate that, despite some changes in perceptions of Cls within the Deaf community, mainstream views of deafness tend to continue to be

\footnotetext{
${ }^{6}$ http://www.deaf.org.nz/about-us/our-vision-values-and-goals

${ }^{7}$ http://www.deaf.org.nz/about-us/our-board-members
} 
grounded in medical views of deafness, and views which authorise the normalcy of hearing. However, in contrast to such views, one participant in Snapp's (2012) study on cochlear implants was a young man who had decided to get implants as an adult. In making this decision, he wanted "access to both orality and to the Deaf world," and wished to "speak Sign language, use his $\mathrm{Cl}$ and live in hearing and Deaf world" (Best et al 2013: 123). My study further contributes to this work, through exploring the narratives of $\mathrm{Cl}$ users on d/Deafness, cochlear implants, identity, and experiences in $d /$ Deaf and hearing worlds. I hope that this thesis will go some way in portraying the experiences of cochlear implants in a New Zealand context, drawing on the voices of those who have firsthand experience of this technology.

\section{METHODS}

This research primarily draws on the narratives, thoughts, and observations of ten participants, which were collected throughout eight interviews. Of these ten participants, four are cochlear implant users, five are parents of cochlear implant users, and one is a teacher of the deaf. The $\mathrm{Cl}$ users were aged between 19 and 23 at the time of interviews, had all left school, and were either at university or in the workforce. The table below outlines some details of all ten participants, as well as one cochlear implant user who I did not interview, but whose parents I did. Whilst the primary focus of this research is on the lived experiences of cochlear implant users, the social and cultural effects of the technology, and its influences on identity, interviewing cochlear implant users, as well as parents and a teacher, allowed me to gain a broader picture of deafness, the technology and associated experiences.

\begin{tabular}{|l|l|l|l|l|l|l|l|}
\hline Pseudonym & Role & Age & $\begin{array}{l}\text { Hearing } \\
\text { status }\end{array}$ & $\begin{array}{l}\text { Cochlear } \\
\text { Implant }\end{array}$ & $\begin{array}{l}\text { Age at } \\
\text { implanta- } \\
\text { tion }\end{array}$ & Education & Occupation \\
\hline Adam & Cl user & 19 & Deaf & $\checkmark$ & 3 & Mainstream & $\begin{array}{l}\text { University } \\
\text { student }\end{array}$ \\
\hline Sandra & $\begin{array}{l}\text { Adam's } \\
\text { mother }\end{array}$ & - & Hearing & $\mathbf{x}$ & - & - & - \\
\end{tabular}




\begin{tabular}{|c|c|c|c|c|c|c|c|}
\hline Hannah & $\mathrm{Cl}$ user & 22 & Deaf & $\checkmark$ & 5 & $\begin{array}{l}\text { Mix of schools for } \\
\text { the deaf and } \\
\text { mainstream; } \\
\text { predominantly } \\
\text { mainstream }\end{array}$ & Working \\
\hline Julie & $\begin{array}{l}\text { Hannah's } \\
\text { mother }\end{array}$ & - & Hearing & $x$ & - & - & - \\
\hline John & $\begin{array}{l}\text { Hannah's } \\
\text { father }\end{array}$ & - & Hearing & $x$ & - & - & - \\
\hline Isaac & $\mathrm{Cl}$ user & 23 & Deaf & $\checkmark$ & 12 & Mainstream & Working \\
\hline Jack & $\mathrm{Cl}$ user & 19 & Deaf & $\checkmark$ & 18 & Mainstream & Working \\
\hline Connor* & $\mathrm{Cl}$ user & 15 & Deaf & $\checkmark$ & $\begin{array}{l}\text { First } \mathrm{Cl}: 3 \\
\text { Second } \\
\mathrm{Cl}: 7\end{array}$ & Mainstream & $\begin{array}{l}\text { High school } \\
\text { student }\end{array}$ \\
\hline Anna & $\begin{array}{l}\text { Connor's } \\
\text { mother }\end{array}$ & - & Hearing & $x$ & - & - & - \\
\hline David & $\begin{array}{l}\text { Connor's } \\
\text { father }\end{array}$ & - & Hearing & $x$ & - & - & - \\
\hline Olivia & & - & Hearing & $x$ & - & - & $\begin{array}{l}\text { Teacher of the } \\
\text { deaf }\end{array}$ \\
\hline
\end{tabular}

*Note: Connor was not interviewed, only his parents were.

I was very fortunate to be able to draw on the contacts of my supervisor Rachel McKee within the Deaf community, especially in that these contacts resulted in setting up the majority of my interviews. I contacted all four cochlear implant users through Rachel, 
making this part of recruitment relatively straightforward. Through these individuals, I contacted one participant's mother, and another participant's mother and father. Another set of parents contacted me through a local support group for parents of deaf children, in which I circulated information about my project. Finally, I contacted a teacher of the deaf through my other supervisor, Catherine Trundle. The relatively small community that I was drawing on for this study lent some difficulties to the recruiting process, as there did not seem to be a large number of people available to be involved in the age group that I was hoping to interview. Despite these limitations, I was extremely fortunate in the contacts I could make through known networks, which, in the end, provided enough participants for a study of this scope.

Interviews with my participants were between one and two hours in length, were conducted in spoken English, were audio recorded, and transcribed by myself. Written consent was obtained from all participants. The interviews took place in a variety of settings, but always at the convenience of my participants. Some were carried out in the homes of participants, while others were carried out in private rooms in a university setting, and one interview was conducted over the telephone. Aside from the interview over the telephone, the interviews with parents were conducted in their homes, whereas the interviews with cochlear implant users were carried out in university settings. Most of the interviews were between one participant and myself; however, when a parental pair was interviewed, they were interviewed together. For the interview with one set of parents, their daughter (who uses a $\mathrm{Cl}$ and had already been interviewed) was also present.

The interviews were loosely structured, consisting mostly of open-ended questions in the hope that these would prompt narratives on topics that were important to the participants themselves. In conducting these interviews, I often began by asking my participants to talk briefly about themselves, their deafness and when they received their cochlear implant. Whilst I attempted to follow the natural flow of conversation and narrative throughout the interviews, I always had an interview schedule with me to ensure that all key questions and topics were covered. Topics discussed in the interviews with my participants ranged from memories before implants, initial experiences of $\mathrm{Cls}$, language, social experiences, friends, education (both school and university), work, involvement in Deaf communities, and hopes 
for the future. Covering these topics, along with others, in interviews allowed me to gain an understanding of my participants' experiences with both deafness and cochlear implants, and therefore understand some of the experiences and social factors that contribute to the identities of these $\mathrm{Cl}$ users. ${ }^{8}$

Speaking with both $\mathrm{Cl}$ users and some parents offered some different viewpoints, and thus interesting insights into the differing ideas on implants, and particularly associated "choices," in the narratives of parents and $\mathrm{Cl}$ users. In particular, interviews with parents served to fill in some of the gaps in the early memories of $\mathrm{Cl}$ users, and provided a chance to learn about the decisions behind choosing implants for their children. Furthermore, I spoke with parents about their hopes and expectations of implants, and how they felt the technology had worked for their children. Whilst these interviews allowed me to gain an idea of the effects that deafness and cochlear implants have had on these families as a whole, they also meant that some more sensitive topics could be spoken about in more depth. For example, parents were in a position to be able to speak, from a more distanced position, about difficulties, especially social ones, that their child had encountered.

Having conducted interviews both with parents alone, and along with their child with a $\mathrm{Cl}$, I can identify positives and negatives of both interview set-ups. It is important to note, however, that these interviews were conducted according to how best these families felt comfortable, or according to what suited them, and not as a result of requests from myself. Interviews with parents alone gave these parents opportunities to open up about the experiences their child had had with deafness and $\mathrm{Cls}$, which might have been more difficult with the $\mathrm{Cl}$ users present. On the other hand, the interview that I conducted with two parents and their daughter provided an opportunity for them to discuss their family's journey with deafness and a $\mathrm{Cl}$ in a way which they would not usually. In discussing the

\footnotetext{
${ }^{8}$ I thus made use of inductive research and semi-structured interviews for this thesis. According to Bernard, a semi-structured interview is scheduled and is "open ended, but follows a general script and covers a list of topics" (2005: 203). In conducting semi-structured interviews, I could make sure that I covered certain topics and areas of the lived experiences of my participants, whilst also leaving room to allow participants to form their own narratives about issues important to them. Induction thus begins with the particular observations, and empirical generalizations and theory building are bottom-up, moving from the data themselves" (Brewer 2000: 108). I have employed an interpretive approach (Geertz 1973) which emphasises the cultural and inter-subjective process of meaning-making that occurs within self-narratives and between the anthropologist and her interlocutors.
} 
interview afterwards with the $\mathrm{Cl}$ user, she told me that the interview had given her a chance to be reassured that they all shared similar views on her $\mathrm{d} /$ Deafness and $\mathrm{Cl}$.

In my analysis of the interviews, however, I was careful not to take the opinions of parents and transpose them onto my $\mathrm{Cl}$ user participants. Accordingly, whilst I draw on the narratives of parents throughout this thesis, I have attempted to focus primarily on what the $\mathrm{Cl}$ users spoke to me about. In saying this, Chapters Two and Three draw extensively on the narratives of parents and their unique experiences in the early stages of their child's deafness and after implantation. Additionally, despite these precautions, the narratives of parents and $\mathrm{Cl}$ users echoed one another on a number of themes, especially those on the outcomes of decisions about $\mathrm{Cls}$, and the choices and opportunities that resulted from these decisions. In this sense, I feel that speaking to both $\mathrm{Cl}$ users and parents strengthens my study, especially in that it gives a voice to those who are most closely associated with the processes involved with cochlear implantation.

In detailing my methods, it is important to recognise my positionality and its influence on my interactions with my participants. As Lila Abu-Lughod notes, the "power of such factors as personality, social location in the community, intimacy of contact, and luck (not to mention theoretical orientation and self-conscious methodology)" always shape ethnographic data collection (1986: 10). Throughout the research process, I struggled with my status as an outsider - someone who is hearing, does not have a cochlear implant, is not a part of the $d /$ Deaf community, and does not speak New Zealand Sign Language - and the effect that this may have had on this project. My outsider status perhaps influenced the degree to which the individuals in my study divulged information about their $d /$ Deaf experiences, and perhaps led these individuals to present certain hearing aspects of themselves to a greater degree. I hope that my status as a young researcher may have countered some of the difficulties of being an outsider, as the cochlear implant users were mostly of a similar age to me. This may have created a more levelled field in terms of the interviews, and put my participants at ease. 
It is important to note here that this study is not representative of the New Zealand population of individuals with Cls, the wider population of people with implants, or the d/Deaf community in New Zealand. Of note, all of the $\mathrm{Cl}$ users in this study have had positive experiences and success with their implants. While these experiences are not misrepresentative, my sample does not include any $\mathrm{Cl}$ users who have had little or no success with their Cls. Despite evidence throughout the 2000s of well-developed speech and listening skills in many young implantees, there continues to be considerable variability in the results of implants (Christiansen and Leigh 2011). Furthermore, some accounts of Cls describe "unpleasant sensations with the implants, causing some to abandon the implants after finding them not beneficial or useful" (Padden and Humphries 2005: 178). I imagine that such individuals may have been more difficult to recruit for this study, and that the experiences of such individuals may be potentially better represented in a separate study.

For the purpose of maintaining the anonymity of the participants in this study, all participants are referred to by pseudonym, and I have omitted both details of where in New Zealand these interviews took place, as well as particulars about places participants spoke about. However, it is important to acknowledge the small size of New Zealand $d /$ Deaf communities, as well as the relatively small number of individuals with implants throughout New Zealand (especially in this age bracket), which, as Fitzgerald et al (2013) note, can make anonymity difficult in studies involving these communities. I have, nevertheless, attempted to protect the identities of all participants involved.

\section{Chapter Outlines}

Following this introductory chapter, Chapter Two lays out the analytical frames for this study - situating it within medical anthropology, Deaf studies and disability studies - and outlines the key themes of identity and liminality. Next, in line with how I began most of the interviews, Chapter Three examines the decisions made about cochlear implants, as well as decisions about education and modes of communication. Chapter Three draws on notions of the 'norm' and medicalised views of deafness in order to explore the underpinnings of the decisions made about $\mathrm{Cls}$, which serve as a catalyst for the decisions that follow. Chapter Four focuses on the lived experiences of the $\mathrm{Cl}$ users, covering practical aspects of both 17 
deafness and having an implant. This chapter highlights the realities of living with deafness and $\mathrm{Cls}$, and, while emphasising the positive aspects of the technology, also counteracts some of the one-sided representations of implants in medical discourse and the media. This chapter links to theories of identities (e.g. Snow and Anderson 1987, Giddens 1991), and explores the embodiment of cochlear implant technology for my participants. Chapter Four also covers the research questions that aimed to explore the experiences of both d/Deafness and cochlear implants. Chapter Five goes on to discuss choices in relation to cochlear implants and the participants in this study. Ideas of choice were emphasised by both parents and individuals with $\mathrm{Cls}$, with both sets of participants stressing that deciding on an implant had resulted in more choices. Chapter Five draws on some modern understandings of choice (e.g. Rapp 1999, Mol 2008), and problematises ideas of 'free' and unrestrained choice, emphasising instead that choices are always in fact influenced by social norms, pressures, and expectations. Chapter Six explores the 'identity work' (Snow and Anderson 1987) of the $\mathrm{Cl}$ users in this study, emphasising their self-narratives and their paths into the $d$ /Deaf world. Utilising theories of identity, identity work, and narrative (e.g. Giddens 1991, Riessman 2008), this thesis seeks to understand how these young individuals have incorporated aspects of both hearing and $d /$ Deaf worlds into their identities. Chapter Seven, then, investigates the liminal position of these $\mathrm{Cl}$ users between $\mathrm{d} /$ Deaf and hearing worlds. This chapter, drawing on theories of liminality (Turner 1969, 1987; Murphy et al 1988, Little et al 1998), explores how these individuals are not fully incorporated into either world. Lastly, Chapter Eight includes recommendations from my participants, along with final conclusions.

Overall, in this thesis I reveal that the identities of the $\mathrm{Cl}$ users in this study are influenced by, and forged through, the multiple threads and social influences of both $d /$ Deaf and hearing worlds. Whilst their cochlear implants influence the formation of their identities, particularly through the embodiment of the technology - and the consequent awareness of the effects and limits of it - the self-narratives of these individuals are influenced by a multiplicity of factors. These factors include medical and familial hopes and expectations, mainstream ideas of the 'normal' body, technological experiences, and individual identity trajectories. Thus, through the formation of coherent narratives, along with identity work, these $\mathrm{Cl}$ users are forging empowered, authentic identities between, and within, d/Deaf and hearing worlds. 


\section{CHAPTER TWO: THEORISING DEAF EXPERIENCES, BODIES AND SUBJECTIVITIES}

\section{INTRODUCTION}

This chapter charts the key theoretical approaches and ideas that I use to understand $\mathrm{Cl}$ users' experiences. First, I show how my analysis is underpinned by the approaches, foci and questions within the fields of medical anthropology, Deaf studies, and disability studies. Whilst these approaches differ from one another, they are able, in conjunction, to contribute to an analysis of the power of ideas of the 'normal,' 'able,' hearing body, and the social systems and influences which impact on bodies that do not fit within such norms. In order to explore my participants lived experiences, I turn to theories of identity, specifically 'identity work' (Snow and Anderson 1987), to understand the processes through which $\mathrm{Cl}$ users form identities, as well as the range of social, cultural, embodied and technological influences that shape the identities of the $\mathrm{Cl}$ users in this study. Given that this research is based on interviews, theories about the reflexive narrative work of identity proved useful for understanding the shape and themes of participants' stories and what mattered most to them in presenting their self-stories. Furthermore, given that a central theme in such narratives was their involvement in two worlds, I show how theories of liminality are an important tool to explore the identity work involved in being in-between, or on the periphery of, established social categories.

\section{UNDERLYING QUESTIONS AND DISCIPLINARY APPROACH}

In situating this thesis within the fields of medical anthropology, Deaf studies, and disability studies, I draw on the "long-standing anthropological awareness that human cultures, bodies, and experiences are generated relationally, developing at the intersections of histories, peoples, structures, and materialities" (Inhorn and Wentzell 2012: 4). Thus, I explore the experiences of $d /$ Deaf bodies with cochlear implants in a New Zealand context, taking into account the social influences on these bodies. Though "many d/Deaf individuals are positive about being d/Deaf, and do not perceive it to be a disability" (Bathard 2012: 3), I 19 
draw on disability studies primarily for its focus on the body, and its interrogation of the binary between able and disabled bodies (Murphy 1990; Shakespeare 2005, 2006; GarlandThomson 2007).

The field of Deaf studies "is comprised of interdisciplinary approaches to the exploration of Deaf individuals, communities, and cultures as they have evolved within a larger context of power and ideology" (Bauman and Murray 2010: 210). Despite the variety of contributing disciplines and hence the variety of perspectives, Bauman and Murray state that "the field's fundamental orientation is derived from the notion that deaf people are not defined by their lack of hearing, but by linguistic, cultural, and sensorial ways of being in the world" (2010: 210). A central tenet of Deaf studies, and therefore of Deaf culture and Deaf identities, is the importance of signed languages. Language and communication therefore represent a key component of deaf experience, and factor into processes of 'identity work,' particularly as language (or language ability) dictates the groups with which an individual may associate. Moreover, language (both spoken and signed) features as a key theme within the narratives of my participants, is a critical factor in the liminal position of these $\mathrm{Cl}$ users, and is often central in cultural identification, and thus self-identity. My research, drawing on Deaf studies, takes a cultural approach to deafness, and seeks to contribute to knowledge on $d /$ Deaf individuals' identities and experiences of being in the world, and the extent to which they draw on $d /$ Deaf and hearing cultures. Despite much progress, $d /$ Deaf identities are still "facing dominant ideologies of normalcy that pigeonhole the meanings of deaf to medical constructions" (Bauman and Murray 2010: 211). This thesis draws on the social model of deafness as a counterpoint to the prevalence and power of these medical constructions. Furthermore, I am challenging predominant medical and mainstream ideas on cochlear implants that often frame this technology as a 'fix' or a 'cure' for deafness, and do so primarily through the voices of $\mathrm{Cl}$ users themselves. This further contests medical constructions of deafness, and promotes a more nuanced understanding of $d /$ Deaf bodies and experiences.

Medical anthropology provides a number of theoretical standpoints that are useful in which to ground this study. According to Inhorn and Wentzell, "medical anthropologists analyse the relations among health, illness, social institutions, culture, and political and economic 
power [...], combining biomedical perspectives with those that address social and cultural problems" (2012: 2). This thesis addresses the $d$ /Deaf body and its material experiences in both $d$ /Deaf and hearing worlds, as well as the realities of $\mathrm{Cl}$ technology for these bodies. Thus, I explore the material biological realities of $d /$ Deaf bodies as they interact and are expressed through culture. Here, I draw on Margaret Lock's notion of 'local biologies,' which

refers to the way in which the embodied experience of physical sensations, including those of well-being, health, illness, and so on, is in part informed by the material body, itself contingent on evolutionary, environmental, and individual variables. Embodiment is also constituted by the way in which self and others represent the body, drawing on local categories of knowledge and experience. If embodiment is to be made social, then history, politics, language, and local knowledge, including scientific knowledge to the extent that it is available, must inevitably be implicated (Lock 2001: 483-4)

Notions of embodiment are useful as, in this study, the biological, material, and technological interact with the social and subjective. In this respect, both local biologies and culture are embodied, and are contingent on individual biology and language, as well as the social, environmental and political contexts in which individuals live (Lock 2001: 484). Consequently, as Lock asserts, "the biological and the social are coproduced and dialectically reproduced, and the primary site where this engagement takes place is the subjectively experienced, socialized body" (Lock 2001: 484). In this thesis, I draw on the ways in which biological or physical deafness is inseparable from the social, cultural and technological aspects of both $d /$ Deaf and hearing social spaces and practices.

\section{THEORISING IDENTITY}

Theories of identity underpin the questions I ask and the interpretive approach that I take in this thesis. I draw on a number of social theorists in order to explore the reflexive identity work of participants. To define identity, I draw here on Snow and Anderson's (1987) theory of 'identity work.' Snow and Anderson distinguish between social identities, personal identities, and self-concept. For these scholars, social identities are "attributed or imputed to others in an attempt to place them as social objects. They are not self-designations or 21 
avowals but imputations based primarily on information gleaned on the basis of appearance, behavior, and the location and time of action" (Snow and Anderson 1987: 1347). Personal identities, on the other hand, "refer to the meanings attributed to the self by the actor. They are self-designations and self-attributions brought into play or asserted during the course of interaction" (Snow and Anderson 1987: 1347). Lastly, Snow and Anderson refer to the self-concept as "one's overarching view or image of her- or himself "as a physical, social, spiritual, or moral being"'” (Snow and Anderson 1987: 1348). For Snow and Anderson, the self-concept is a type of "working compromise between idealized images and imputed social identities" (1987: 1348). These three concepts often interlink with one another, as what others think of us tends to influence our sense of self. Drawing on these definitions, Snow and Anderson call the process of identity construction and assertion 'identity work,' by which they refer to "the range of activities individuals engage in to create, present, and sustain personal identities that are congruent with and supportive of the selfconcept" (1987: 1348). It is important to note that identity is seen as an ongoing process, rather than a fixed quality, and is subject to change over time. In this thesis I focus on the ongoing process of identity work by examining the ways in which the $\mathrm{Cl}$ users are working to establish an authentic sense of self, and variously incorporating different elements of their subjective and social experiences into their identities.

An important issue emphasised within the concept of identity work is the socially mediated nature of subjectivity. Smiler and McKee state that "[c]ontemporary models of identity posit that identity is not a fixed set of personal and social characteristics," but instead results from processes of interaction between people, institutions and practices (2006: 96). Accordingly, as Leigh et al emphasise, identity constructs "are rooted within a societal framework, meaning that they evolve out of various social experiences. The experiences incorporate not only the self-evaluation of the individual but also the evaluation of others, as well as opportunities and constraints regarding self-definition imposed by those others doing the evaluation" (1998: 329). Accordingly, one's identity is not formed internally and independent of outside forces, but is instead reflective of one's social positioning and social interactions. The evolution of identity through various interactions with others is a "multidimensional, reflexive process involving psychological motivation, cultural knowledge, and the ability to perform appropriate roles" (Maxwell-McCaw et al 2000: 2). For the $\mathrm{Cl}$ users in my study, their sense of self was influenced by their various relationships, interactions and 
ties with both d/Deaf and hearing people - including, centrally, family, friends, peers, doctors and teachers. Furthermore, these were reflective, at times, of how they wished to be viewed, or evaluated, by others.

\section{NARRATIVES OF IDENTITY}

The narratives of our life experiences that we construct for ourselves and for others help to build our self-concepts and, accordingly, are important aspects of identity work. I thus draw out narrative themes throughout this thesis. Anthony Giddens (1991) describes the development of identity in modernity as a reflexive project, in which "self-identity is constituted by the reflexive ordering of self-narratives" (1991: 244), and consists of sustaining "coherent, yet continuously revised, biographical narratives" (1991: 5). Therefore, according to Giddens, one's identity "is not to be found in behaviour, nor [...] in the reactions of others, but in the capacity to keep a particular narrative going [emphasis in original]," and so one must "continually integrate events which occur in the external world, and sort them into the ongoing 'story' about the self" (1991: 54). Catherine Kohler Riessman suggests that the importance of crafting reflexive self-narratives is due to the fact that identity is no longer viewed as fixed, given and "natural" (2008: 7). Instead, "individuals must now construct who they are and how they want to be known [...]. In postmodern times, identities can be assembled and disassembled, accepted and contested, and indeed performed for audiences" (Riessman 2008: 7). For the young d/Deaf people in this study, the advent of new technologies has created new options and choices for crafting selves, and thus for shaping self-narratives. In this respect, $\mathrm{Cl}$ technology has enabled these individuals to draw on aspects of and experiences in the hearing world, whilst they also remain aware of their d/Deaf body and experiences in the d/Deaf world. In turn, these individuals have to successfully incorporate these various and differing experiences into a coherent self-story.

It is important to reference the work on narratives in medicine and health in relation to this thesis, especially given the research on illness narratives within anthropology (Kleinman 1988, Mattingly and Garro 2000, Trundle 2011). This work contributes to our understandings of cultural narratives about the 'normal' body and somatic disruption. According to Arthur Kleinman, "[i]llness narratives edify us about how life problems are created, controlled, 23 
made meaningful. They also tell us about the way cultural values and social relations shape how we perceive and monitor our bodies, label and categorize bodily symptoms, interpret complaints in the particular context of our life situation" (1988: xiii). Furthermore, he states that

patients order their experience of illness - what it means to them and to significant others - as personal narratives. The illness narrative is a story the patient tells, and significant others retell, to give coherence to the distinctive events and long-term course of suffering. The plot lines, core metaphors, and rhetorical devices that structure the illness narrative are drawn from cultural and personal models for arranging experiences in meaningful ways and for effectively communicating those meanings (Kleinman 1988: 49)

Consequently, through the use of narratives, individuals construct their identities with reference to their experiences with their bodies, medical technologies, medical labels and illness. Although my participants' narratives are not presented as illness narratives, they are centred on the body, and how it may be culturally or medically categorised due to its d/Deafness. In this thesis, I attempt to show that the narratives of the $\mathrm{Cl}$ users both sit within, and seek to challenge, mainstream ideas of $d /$ Deaf bodies and the medicalisation of deafness, especially through cochlear implants.

I also make use of ideas about the fluidity of identity. In their narratives, my participants reflexively formed fluid identities that incorporated a number of different aspects from d/Deaf and hearing social worlds and cultures. I draw on Stuart Hall's work on identity, in which he argues "that identities are never unified and, in late modern times, [are] increasingly fragmented and fractured; never singular but multiply constructed across different, often intersecting and antagonistic, discourses, practices and positions. They are [...] constantly in the process of change and transformation" (2000: 17). Likewise, the types of identities a $\mathrm{Cl}$ user claims and develops may vary depending on which social world (d/Deaf or hearing) they are involved in at any one time, and the extent to which they identify with their deafness. Foster and Kinuthia (2003) developed a model in which identity is constructed through four factors: individual characteristics, situational conditions, social conditions, and societal conditions. Together, "the four factors act in combination, to 
produce a fluid, dynamic, sense of identity in which one or more of the individual characteristics is selected, mediated, and drawn out in a response to a particular situational, social or societal condition" (Foster and Kinuthia 2003: 279). Whilst their model is based around the interactions of Deaf identities and ethnic identities, Foster and Kinuthia's assertion about the combination of these four factors can be seen in the identities of $\mathrm{Cl}$ users, as I will demonstrate in this thesis.

Additionally, I draw on some concepts of d/Deaf identity, which have been explored reasonably widely within the literature on d/Deafness (see Glickman 1996, Leigh et al 1998, Maxwell-McCaw et al 2000, Smiler and McKee 2006, Davis 2007, Brueggemann 2009, Leigh et al 2009). As the majority of deaf children are born into hearing families, they are more often socialised into hearing culture than Deaf culture. Thus, "deaf identity is not necessarily a core identity for deaf children who grow up within hearing families. Instead, incorporating a deaf identity into one's self-image is a developmental task that is influenced by family and school variables" (Leigh et al 2009: 246). Identity formation can therefore be tension-filled and confusing for deaf people, especially as they are growing up. Leigh et al state,

deaf individuals with hearing parents may face particular struggles with identity development, particularly if they have been trained to identify with hearing values and become aware of a Deaf culture at later stages in their lives. This would introduce an element of competition with the culture of their hearing parents due to inherent deaf/hearing conflicts that can emerge until one learns to comfortably navigate both Deaf and Hearing cultural paradigms (1998: 337)

I argue that these difficulties in identity development may be accentuated by cochlear implants, especially in contrast to the expectations that $\mathrm{Cl}$ users will be able to seamlessly integrate into the hearing world. The creation of what seems to be an artificial hearing identity appears to "run the danger of creating "outsider" status for some cochlear implantees, who can never fully be a part of hearing culture due to the technical limitations of implants in replicating normal sound, nor of Deaf culture due to the focus on auditory sounds and spoken language that typically accompanies implantation" (Leigh and MaxwellMcCaw 2011: 102). Whilst d/Deaf identities have been well-researched, there has been less 
work done on the identities of $d$ /Deaf individuals with cochlear implants; thus, I see my thesis as adding to this literature.

Given that the $\mathrm{Cl}$ users in my study were aged between 19 and 23 at the time of interviews, it is important to note the formative place of adolescence in identity development. Sheridan states that, "[a]s cognitive abilities mature, the self-concept becomes increasingly more abstract, which allows adolescents to better differentiate their sense of self and identity from that of others. Adolescents grow in their ability to reflect on their psychological characteristics, their understanding of themselves, and their fit in various situations" (2008: 19). In Western society, adolescence tends to represent the beginning of a life-long process of identity development, in which one begins the complex task of "[i]ntegrating one's past, present, and future into a cohesive, unified sense of self" (Tatum 1997: 20). Consequently, this stage in my participants' lives represents a particularly important phase in the integration of aspects of both $d /$ Deaf and hearing social worlds into their identities. It is in this stage, through reflexively ordering their self-narratives, that they appear to be working through where they fit in relation to each of these worlds, and where they want to establish themselves socially. It is of particular importance for the $d /$ Deaf aspects of their identities as all of my participants were exposed to d/Deaf social groups or communities, and re-exposed to sign language, at various stages in their adolescence. Given that this stage of identity formation is a time of flux and testing, I conceptualise the $\mathrm{Cl}$ users in this study as being in a liminal state.

\section{LIMINAL IDENTITIES}

To further explore identity, I draw on theories of liminality - the state of being 'in-between' - particularly in relation to fluid identities, social boundaries and biculturalism. Arnold van Gennep (1960) first proposed the theory of liminality, suggesting that it was composed of three stages: the separation of an individual from society, a liminal phase, and the reintegration of the individual into society. Later, Victor Turner (1969) further explored van Gennep's ideas of the liminal phase of the ritual process. According to Turner, 
The attributes of liminality or of liminal personae ("threshold people") are necessarily ambiguous, since this condition and these persons elude or slip through the network of classifications that normally locate states and positions in cultural space. Liminal entities are neither here nor there; they are betwixt and between the positions assigned and arrayed by law, custom, convention, and ceremon[y] (1969: 95).

In further developments of the theory of liminality, Turner asserts that the liminal is characterised by a peculiar union: "that which is neither this nor that, and yet is both" (1987: 9).

Within medical anthropology, theories of liminality have been employed in explorations of illness (Little et al 1998), and of severe physical impairments (Murphy et al 1988). Murphy et al (1988) characterise physically impaired individuals as liminal, rather than deviant, in order to explain the way in which they are separate and apart from mainstream society. Thus, liminality is not necessarily a tripartite process in which individuals pass through neatly defined phases. While $\mathrm{Cl}$ users often don't think of themselves as ill or disabled, these theories are useful for thinking through how cochlear implant user participants find themselves in between $d /$ Deaf and hearing cultures, despite their involvement in both. Whilst they may be accepted in, and a part of, both worlds, I will show how it can be difficult for $\mathrm{Cl}$ users to be fully part of one world or the other. Accordingly, they elude categorisation as either d/Deaf or hearing, and, though perhaps poised to enter either world, are outside the boundaries of each (Murphy et al 1988). In turn, these individuals may experience tension in terms of their sense of belonging in either world, and therefore experience alienation from their sense of self (Little et al 1998).

Additionally, I employ liminality in order to further understand the place that deaf bodies occupy in relation to ideals of the 'normal' body in society. In this respect, deaf bodies are seen to be disabled, and thus representative of abnormality. The disabled, or deaf, body becomes "a counterpoint to normality" (Murphy 1990: 117), and is relegated to the periphery of society, positioned outside of the mainstream. As Little et al argue, the era of biomedicine and biopower has introduced a new understanding of both illness and 
disability, in that these states are "no longer an episode in normality, but an abnormality in a life trajectory which is supposed to be smooth" (1998: 1492). Deaf individuals, then, may incorporate their position outside of mainstream society into their identity, and, through identity work, strive towards an alternative belonging in $d /$ Deaf worlds, a space in which they may also not quite fit or identify. While I see my participants as occupying an 'inbetween,' peripheral state, it is important to note that they are not necessarily liminal at all times as, depending on situational, social or societal conditions, these individuals may indeed be incorporated into one of these worlds at any one time. I will thus show the dynamic nature of liminality for $\mathrm{Cl}$ users.

\section{CONCLUSION}

In this thesis I draw on medical anthropology for its nuanced understandings of the relationship between the biological and the social, whilst disability studies offers critical theories on the dis/abled body and the effects of social and medical normalisation processes. Additionally, scholarship drawn from Deaf studies sharpens my focus on d/Deaf people's own subjective experiences. To explore these themes, I draw on theories of identity work and self-narratives to reveal the ways in which $\mathrm{Cl}$ users navigate identities in diverse settings, and utilise ideas of liminality to understand a central theme of their narratives how they navigate living 'in-between' d/Deaf and hearing worlds. 


\section{CHAPTER THREE: DECISIONS}

\section{INTRODUCTION}

The decision about a cochlear implant tends to be the first in a series of key decisions for a deaf individual and his or her family, with consequent decisions predominantly surrounding language and education. All of these decisions tend to centre on the hearing, mainstream world. From early on, hearing parents of deaf children tend to be confronted with a medicalised view of deafness and are presented primarily with ways in which to 'treat' their child's deafness. This is a reflection of the importance of normalised, perfect bodies in contemporary society (Rapp 2001, Garland-Thomson 2007), as well as biomedical notions of curing or fixing deaf bodies in order to return them to normality. Therefore, parents often become focused on correcting the deafness of their child, an action which is socially sanctioned by wider society (Lane et al 1996, Landsman 2009). Parents may become invested in medical trajectories for their deaf children in the face of modern Western expectations that children will live better lives than their parents (Lane et al 1996), and so that their children can be involved in their own mainstream, hearing culture. Therefore, ideas of impairment become "central to all the choices relating to the [deaf] child's future that confront the family," with professionals citing that "a child with a loss of a bodily function has an impairment, the impairment gives rise to a disability, a severe restriction in a normal human activity, namely, communication; and the disability handicaps the child, preventing him or her from fulfilling various social roles" (Lane et al 1996: 34). Thus, whilst decisions about communication and education are not overtly medical, they are made within medical or pathological frameworks, often in the hope of normalising or mainstreaming a deaf child.

Given the increasing options and opportunities available to deaf children in the $21^{\text {st }}$ century, their parents and caregivers tend to have a "host of choices thrust upon [them] in the first few months of their child's life" (Mitchiner and Sass-Lehrer 2011: 71). In particular, these decisions have increasingly revolved around different types of technology, a reflection of the process of what social scientists term medicalisation (Conrad 1992, 2005). Medicalisation 
involves "defining a problem in medical terms, usually as an illness or disorder, or using a medical intervention to treat it" (Conrad 2005: 3). Medicalisation is interesting here in that, given the rise of technology available for deaf individuals, both parents of deaf children and deaf people themselves are increasingly making decisions about medical intervention, rather than being exposed to choices in which accepting deafness and using sign language are viable options.

\section{Technology and Normality: Decisions About Cochlear Implants}

In a New Zealand study on the decisions made by hearing parents of deaf children about cochlear implants, Snapp (2012) discusses how these parents did not see their choices about Cls as 'decisions.' Instead, they "assumed that the best thing for their child was that the child should be given access to normal hearing" (Snapp 2012: 7). Consequently, these parents "described their decision to proceed with a cochlear implant for their child as unproblematic and straightforward" (Snapp 2012: 15). Similarly, in a British study which involved interviews with 216 parents of $\mathrm{Cl}$ users, "[t]he majority of parents reported that they had found the decision over implantation relatively straightforward, as they believed their child had nothing to lose and everything to gain. There was 'really no alternative'" (Sach and Whynes 2005: 402). In some respects, the parents in this study also appeared to come to their decisions about $\mathrm{Cls}$ in a relatively straightforward manner; however, they tended to cite more nuanced reasons for doing so. As will be discussed in Chapter Four, my parent participants drew on notions of choices and opportunities throughout their narratives on choosing $\mathrm{Cls}$ for their children. The notion of straightforward decision-making in the responses of parents from both Snapp's (2012) study, and the study by Sach and Whynes (2005), raises questions as to the distinction between choices and decisions, and the extent to which choices are in fact 'free.' Rayna Rapp, in her study on the social impacts of amniocentesis in America, states that "[b]ecause "choice" is market-driven in contemporary North American culture, it appears to be based on expanding possibilities. But at the same time, available options are shaped by complex forces that travel as the "invisible hand" of the market" (Rapp 1999: 37). In the case of cochlear implants, such forces may include the dominance of medical interests, and the influence of normality in society. 
Adam and Hannah, who were three and five years old, respectively, when they were implanted, had no input into the decisions to receive a cochlear implant; instead, it was their parents who made these decisions. Adam and Hannah's parents primarily received information on Cls from Advisers on Deaf Children (hereafter AODC), ${ }^{9}$ but supplemented this through their own personal research. Julie and John, Hannah's parents, first found out about Cls when Hannah was four years old, and the process progressed reasonably quickly once they realised that a $\mathrm{Cl}$ was an option. They first received information about $\mathrm{Cls}$ from AODC, who also provided them with other information in the early stages after Hannah's deafness was diagnosed. John noted, "It was their job or their role [advisors] to look at all the options and advise parents and let parents make the choice." So, from early on, advisers acted as an important source of information for Julie and John. It is interesting to consider, however, whether parents are able to gain information about all options regarding the way forward for their deaf child through a service such as AODC. In this respect, and given that parents tend to have already been ushered onto a medical trajectory in terms of dealing with their child's deafness, parents may not have access to much information on accepting their child's deafness and using sign language. In terms of their ultimate decision for Hannah, Julie recalled, "So really when we heard about it [cochlear implants] we investigated it, and thought, 'goodness we couldn't think of a reason why you wouldn't do it.'" Having tried hearing aids for Hannah unsuccessfully, Julie and John decided to opt for a $\mathrm{Cl}$, and Hannah was implanted when she was five years old. According to their narratives, then, Julie and John's decision about a $\mathrm{Cl}$ for Hannah was relatively straightforward, with a backlash from some members of the Deaf community the only deterring factor.

Sandra, Adam's mother, also found out about cochlear implants through an AODC, along with information about education and the needs of deaf children. She noted that the AODC was the first point-of-contact in the support system for her and Adam. This support system is likely to further notions that deafness is a disruption to normality and that systems need to be put in place to manage it, with parents' decisions about Cls further influenced by such considerations. Whilst Sandra received information from the advisor, who she found "really supportive" and informative about the options for Adam, she was also careful to collect

\footnotetext{
${ }^{9}$ Advisors on Deaf Children are a service provided by the Ministry of Education, who "co-ordinate services and advise on appropriate programmes to meet the communication and learning needs of the students" (New Zealand Ministry of Education 2014).
} 
information herself, in an attempt to be fully informed and to make the best decision for Adam. It is interesting to note, however, whether decisions can be 'fully informed' given the complex forces that are implicitly present in decision making (Rapp 1999).

Sandra: I just gathered the information and [...] thought it through and worked at every angle, and of course with Adam, it was just really a situation that his family was in, [...] I was a single mum with him, his extended family was in [another town], [there] was not a very good relationship with his father, none of his [dad's] family would sign. [...] My sister and my mother did because they're teaching-based, but I could just see that l'd have to make a choice that l'd move somewhere, to a [deaf] school, and van Asch ${ }^{10}$ was it for Adam, or had to really consider whether I could give him another option apart from signing, so that he could really work in both areas for support, 'cause it was going to be very challenging if he just signed to me in [hometown]. And remember at that stage I wasn't being supported in New Zealand Sign as an option, at all, [so I] weighed everything up, [and made a] decision.

As is seen in Sandra's narrative, her decision about a $\mathrm{Cl}$ for Adam was influenced by family and their willingness to accept and work with Adam's deafness, as well as his place in the social world. She believed that Adam's social world would be limited if she did not choose an implant for him and work for him to use spoken language. This demonstrates the lack of networks for young deaf people in New Zealand, and the accompanying importance assigned to involvement in wider hearing society. In making her decision about a $\mathrm{Cl}$ for Adam, Sandra also considered communication and education options. She thought carefully about giving Adam the opportunity to be supported in both New Zealand Sign Language and English, and the ways in which an implant might give him these options. However, she received little support in her desire to give Adam access to NZSL as Signed English ${ }^{11}$ was the supported mode of sign language for deaf children at the time. Thus, in this case, veering

\footnotetext{
10 "The van Asch Deaf Education Centre is a residential special school for deaf children, and is situated in Sumner, Christchurch, New Zealand" (van Asch Deaf Education Centre 2008). It is one of two Deaf Education Centres in New Zealand, the other being Kelston Deaf Education Centre in Auckland (Smith 2003).

${ }^{11}$ Signed English uses signs in an English language structure (Lane 1999), whilst NZSL follows its own structure and is the language of use in New Zealand Deaf communities.
} 
from the norm was unacceptable in managing deafness and Sandra was offered little help after this decision. After weighing up this lack of support, along with the small size of their hometown and the limited social opportunities that this offered, Sandra made the decision to have Adam implanted when he was three years old. To an extent, Sandra's narrative on her decision making process reflects wider difficulties in raising a deaf child within a hearing environment: the lack of certain resources, the pressure to conform to hearing norms, and the accepted ways of managing deafness.

Once Anna and David's son, Connor, was diagnosed as profoundly deaf, they decided that a cochlear implant might be the right option for him. However, they encountered a number of obstacles within the healthcare system, and became frustrated in the process of getting an implant for Connor. Anna and David (unlike Julie, John, and Sandra) did not receive much information on cochlear implants from other sources, and felt, instead, that they had to fight for access to information and approval for a $\mathrm{Cl}$, as well as support. They spoke about the disorganisation and unprofessional nature of the services that they encountered when Connor was diagnosed, and that they were presented with a huge amount of conflicting information about the options for managing Connor's deafness. Anna also noted that they "didn't actually get much positive stuff about it [cochlear implants]. Initially we were told by his first audiologist [...] to shy away from it. [She/he] didn't encourage it at all. We had to pretty much do our own research." In a basic Google search of cochlear implants, the majority of information returned adheres to a medical model of deafness, and is largely positive about $\mathrm{Cls}$ in their quest to 'fix' deafness. Therefore, the information that parents may find in their own research furthers ideas of cochlear implants as a socially acceptable way in which to manage deafness. In a reflection of the sometimes inaccessible nature of the medical system, Anna noted that medical professionals "used language that was quite difficult to [understand] - you know, like it's alright for me 'cause I'm a doctor - but for a lot of lay people, this language was quite [...] complex, and they didn't really explain it in terms that were meaningful for non-medical trained people." This highlights the fraught nature of the medical system and the difficulties that many face in accessing health services. Thus, Anna and David engaged in a long process of gathering information about $\mathrm{Cls}$ and fighting to see the cochlear implant team in Auckland. After a first assessment with $\mathrm{Cl}$ services resulted in stronger hearing aids for Connor, Anna and David insisted on a reassessment as they did not believe that the aids were making a difference. On this, Anna stated, "I had to beg to go 33 
to Auckland to be seen by the implant team again, and then they said, 'yes we do agree with you - we do think he should have an implant."' Anna and David encountered difficulties in their navigation of a complex national health system with limited resources, especially in that they were dealing with multiple services in managing Connor's deafness. Connor was implanted at age three and, unlike the other families in this study, Anna and David decided on a second implant for Connor, which he received when he was seven years old.

As opposed to the participants whose parents made the decision about their implant, the decision was largely left to Jack and Isaac given their ages when implanted. Isaac received his $\mathrm{Cl}$ when he was 12 years old, and was given primary responsibility for the decision: "my parents were pretty supportive of the whole idea that I get to pick my own future I guess, so they made sure I was well informed, and made sure that I knew what was going on, but left the ultimate decision down to me." In this respect, through encouraging Isaac towards individual decision making, his parents were attempting to self-responsibilise him. Such efforts are reflective of the value of creating an independent self in New Zealand society, who is thus empowered and individualised. Jack, who was 18 years old when he was implanted, spoke about how he gathered information on implants and making his decision:

[P]robably a little bit of self-research [to get information on cochlear implants], a little bit of YouTube clips. And I could see there was both positivity and negativity towards it, and also the health risks, but in the end, because I could just see that there are more people that had, I don't know, experienced a more positive outlook on life, because they could [...] interact more with their friends and stuff. Because l'd started to notice that I was withdrawing a bit more, because I was a bit scared of missing things, so it was a big factor, because I knew that if I didn't take any action, then my whole like teenage [years] and like all my relationships would just, would eventually decline, and so I didn't want to do that, so I made sure that I took some steps to help with that.

From Jack's comments, it is clear that a consideration of adolescent social connections played an important part in his decision making process. His deliberation over these connections demonstrates the importance of peers, especially for someone in their teenage years like Jack, in the formation of one's identity in New Zealand. For Jack, his peer 
relationships contribute to his sense of belonging in the world. With his level of hearing declining, he began to lose a sense of his self, and began to distance himself from his social relationships. This is perhaps reflective of his increasing inability to pass as hearing and get by in the hearing world, along with both his own and others' discomfort with such a move away from hearing norms. Accordingly, he took it upon himself to remedy this and to ensure continued belonging in his social groups. The importance of being able to associate with his current social group, and his efforts to sustain his own, and others', image of himself, represents his continual engagement in 'identity work' (Snow and Anderson 1987). Furthermore, his decision to get an implant enabled Jack to continue his trajectory of development (Giddens 1991), as he hoped to remain involved in the social worlds which he knew and to which he belonged. Whilst Jack's own decision was informed and well thought through, he emphasised in another section of his narrative how doctors may present parents and potential implantees with biased information on the technology and its outcomes.

Olivia, a teacher of the deaf, who has previously worked in North America, also spoke about the importance of parents being informed when making a decision about $\mathrm{Cls}$.

I think that's probably where the conflict is, is treating this as something that needs to be fixed and perhaps information not being presented. If you say to a parent, 'the outcome is 90 per cent successful,' as a parent, 'fantastic, sign me up!' But without clarifying [that] it's a lot of money to be made and [...] I think there's just layers of complication to how those decisions are made and who does the informing. [A]s a parent who's probably never seen a deaf person you're going to defer to a doctor, you know you're going to trust the professional in front of you and so I would say [...], I don't know that I've seen nearly as much of [...] what I would call inappropriateness here as in [my home country], but I mean it was very prevalent [there], certainly where I was, where, 'oh well it's fine, just put the cochlear on and everything's fine.' Well that's not at all what happens [laughs]. [l]t's not like you just pop the magnet on and everybody hears, [...] like normal hearing, it takes a long time and a lot of [...] work [...] and not just for the kid, for the family [too]. 
Amongst my participants - particularly the $\mathrm{Cl}$ users and Olivia - there was concern over misinformation, or perhaps selective information, being provided to parents when they are making decisions about implants for children who are deaf (see Blume 1997, Hyde and Power 2006, Hyde et al 2010). This demonstrates the power of the medical world in modern society. As Lane et al state, "[m]edical science is revered in our society and is well-funded by government and industry," whilst "the DEAF-WORLD is little known, and there is no one to promote and protect its values and views except Deaf people themselves and their hearing allies" (1996: 371). Thus, though it is important to acknowledge that medical science is dedicated to improving people's lives, "doctors and other professionals have commonly locked Deaf people out of the councils where decisions vitally affecting them are made" (Lane et al 1996: 371).

\section{'The Limits of my Language Mean the Limits of My World' ${ }^{12}$ : The IMPORTANCE OF (SPOKEN) LANGUAGE}

Underpinning decisions about cochlear implants sit decisions about communication. Whilst these decisions were not always explicitly posited as the primary concerns of participants it appeared to be taken for granted that the $\mathrm{Cl}$ users in this study would learn to speak English, given that they had been implanted. It is important to acknowledge the significance of these decisions and the influences that they have had on the lived experiences of the participants in this study. When they discovered their child was deaf, some of the parents in this study decided to use some form of sign language. ${ }^{13}$ However, once the deaf individuals were implanted, this tended to drop away, whether this was intentional or not. As the participants with cochlear implants reached their teenage years, however, all four took up NZSL at various stages and in various ways. This is reflective of wider trends amongst deaf people who find Deaf culture and sign language as they grow up, often once they have left school and have access to more deaf people or an established Deaf community (Lane et al

\footnotetext{
12 A quote from Ludwig Wittgenstein, an Austrian-British philosopher (Martland 1975).

${ }^{13}$ This was not always New Zealand Sign Language (NZSL) as the language was not widely taught within the education system when the individuals with Cls in this study were born. Signed English (sign language which uses the same grammar as and matches each word of spoken English) and Total Communication (which makes use of a number of modes of communication at once, e.g. signed and oral language) may have been presented as options to parents instead.
} 
1996, Skelton and Valentine 2003, Smiler and McKee 2006). Furthermore, it represents the central role of sign language in Deaf identities. All of the participants with $\mathrm{Cl}$ in this study were born to hearing parents and tended to be brought up with the hope of attaining spoken language so they could participate in their parents', and the dominant, hearing worlds. These hopes reflect those of the parents in Snapp's study with hearing parents of deaf children, who "felt that in order for their children to fit in, to attend school and to live full lives they needed to be in a position to have access to the hearing world via the acquisition of spoken language" (2012: 48). The extent to which the families in this study have embraced sign language as an alternative, or supplement, to spoken language for their children varies, and is explored below.

Sandra wanted Adam to have access to both spoken English and NZSL, believing that this would be the best option for him, especially in giving him the opportunity to participate in both $d /$ Deaf and hearing worlds. Sandra believed it was important that Adam, as a deaf child, had access to NZSL from an early age. However, given its limited availability at the time and in their locale, and the prevalence of Signed English, Sandra was subjected to some negative reactions.

[...] once they've veered off the path of the norm, like the expectation was that Adam would learn English Sign, you know the language that was given to the kids from an educational perspective, not New Zealand Sign - once I veered off from that and said that I wanted my child to learn the proper deaf language, 'cause he's deaf, then I fell a little bit foul of everybody [education professionals, particularly at van Asch]. No one really wanted to be that helpful.

Despite coming up against obstacles in access to NZSL, Sandra enlisted the help of a deaf woman and attended night school classes to learn the language and to be able to teach it to Adam. With the use of NZSL, Sandra and Adam could communicate before he was implanted. Some decisions are thus harder to make than others. Due to social pressures and expectations, certain choices will involve more work, and have less institutional support available (a theme I take up in Chapter Five). 
In making the decision about a $\mathrm{Cl}$ for Adam, Sandra carefully weighed up the importance of being able to communicate with all family members. In this sense, Sandra believed that a $\mathrm{Cl}$ would enable Adam to communicate with hearing people using spoken English. Therefore, in choosing an implant, Sandra was hoping to give Adam the opportunity to access spoken language in addition to the signed language he was already using. At school, Adam was supported in NZSL through a teacher's aide, but when he reached Year Three, his mainstream teachers recommended that Adam focus more on his speech. Such favouring of spoken language over signed language is reflective of wider trends (Padden and Humphries 2005), with $\mathrm{Cl}$ users often deprived of access to sign language after implantation, on the advice of medical and educational professionals (see Lane et al 1996, Lane 1999). On this transition to a focus on spoken language for Adam, Sandra said,

[...] he got to about, I think six or seven, and his speech was quite slow, like he'd had a, he'd gone through good stuff, and then he started to slow a bit, and she [teacher aide] was very, very pro New Zealand Sign and was really pushing him hard that way, and the teachers at his school felt that to the point he wasn't paying attention to the words like they kind of felt that, yes his signing's good, but it's slowing him down, he's just trying to, instead of looking and listening and trying to work out the words, he was looking to her to interpret all the time, and, which is good in one way, but because we'd gone in the way of the cochlear implant to help his speech, that must have been, if she didn't sign, and he had to really knuckle down and do it, that that would be better for him. And I remember at the time, with the decision making, I was very adamant that we can't take it away from him, he needs to sign, and the teachers were like, that's fine, but look, he's just got so much potential that he's not going to reach at this rate, and it's not going to suit in a mainstream school. That's when we just felt that would be a bit better, so I kind of went with the school, and thought, well you know, he does need to pick it up [...].

Sandra's narrative highlights that her wish for Adam to be able to use both NZSL and spoken language was difficult to achieve, as it was believed within the education system that one inevitably had to take priority in his language development. In this respect, Adam was embedded in educational systems which did not value bilingualism in spoken and signed 
languages (or perhaps struggled to provide adequate resources for both), and which emphasised the importance of speech. At this stage, as Sandra expressed, Adam "learnt to communicate more with other people that didn't sign. I think that was, he expanded his horizons in other ways but reduced that side [sign language], which was probably, in hindsight, unfortunate for Adam, but I wouldn't have been able to supply everything to him." In this sense, Sandra felt that, as a hearing parent of a deaf child, she could not expose Adam to Deaf culture as much as she would have liked. Thus, Sandra's narratives reinforce that she made the 'right' decisions given the situation, and that these decisions were meaningful for Adam and herself (Riessman 2008).

For other parents, support for NZSL was easier to access, which may be a reflection of geographical location and ease of access to the deaf education system. When John and Julie learnt of Hannah's deafness, they became involved with Kelston Deaf Education Centre, sending Hannah to the preschool and learning NZSL themselves. At the time, sign language was their only means of communication, and therefore an important aspect of family life. Once Hannah was implanted, John and Julie still used sign language with her.

Julie: [...] and one thing that, from her implant that I have never done which they [audiologists] recommended when she first got it, was to take, stop signing and make her use it, I don't agree with that.

John: We didn't do that.

Julie: We didn't do it because I think it's, I think it's cruel. And the fact is I don't see that a cochlear implant makes anyone hearing, it just makes them deaf with skills. So we always kept signing to her, much to everyone's horror [laughs], in secret.

Maintaining sign language after implantation was particularly useful in teaching Hannah spoken language, as she had had no access to sound previously; and they could sign the meaning of spoken words. Adam and Sandra spoke of similar experiences to Hannah and her parents, in that sign language proved a useful tool in acquiring spoken language. At the same time, Hannah had intensive lessons with speech therapists, which continued for a number of years. Whilst she had some access to sign language at school until intermediate-age, spoken language eventually became the family's focus. Thus, as is seen in the cases of Adam and 39 
Hannah, sign language is often perceived "as an ancillary, transitional tool for accessing the curriculum and English rather than as a native language for mediating learning and social identity" (McKee 2008: 535-6). In choosing a Cl for Hannah, Julie and John were keen that she also talk and hear, rather than only use sign language. This is reflective of the fact that common notions of progress and success in relation to Cls "all relate to the development of spoken language skills" (Swanwick and Tsverik 2007: 217). Once again, such a focus on spoken language reveals the importance of hearing and speech as markers of normality and ways in which to transcend the limitations of deafness.

Before Anna and David's son, Connor, aged 15, was implanted, he used hearing aids reasonably successfully which meant that he had some access to sound and language. Accordingly, Anna and David have focused largely on spoken language, and have not used much sign language. Anna spoke of the time and effort that they put into developing Connor's language skills, and the success of such perseverance. In this respect, for Anna and David, Connor's production of language is emblematic of success. Furthermore, their narratives highlight that their decisions about both hearing aids and implants were 'good,' and therefore meaningful in their lives and in Connor's (Riessman 2008).

Anna: [...] one of the reasons he did so well was because we had absolutely been anal about him wearing hearing aids. [...] every single waking moment that we had, he had his hearing aids in, and we were speaking to him. So when the implant did go in, [...] he actually already had quite a lot of language, in his brain. [...] he actually was stringing three words together, three or four words together when he was three, yeah he was. 'Cause some children aren't sort of saying much, but he was stringing, he was making a sentence. So he had a lot of rich language, and that just, it just exploded when he had his implant put in, just you know, all mushroomed out.

Anna and David wanted Connor to be able to access speech, to engage with the hearing world, and their decision to have Connor implanted aided in these goals. Such goals facilitate the proliferation of medical models of deafness, in which parents' decisions tend to centre on deafness as impairment and the ways in which this impairment can be remedied (Lane et al 1996). Furthermore, Anna and David's focus on speech reflects wider trends amongst 
parents who choose Cls for their children, in that the development of speech is a major factor in these decisions (Hyde et al 2010).

\section{Mainstream Desirability: Decisions About Education}

The current educational contexts for deaf children in New Zealand include services provided by the two Deaf Education Centres, Kelston Deaf Education Centre in Auckland and van Asch Deaf Education Centre in Christchurch, some services provided by the Ministry of Education, and education within mainstream settings (Smith 2003). The Deaf Education Centres have reduced in size over the last three decades, leading to redefinitions of their roles. These two Centres provide four different types of services: residential base schools, which are now relatively small; deaf resource classes, which "consist of groups of deaf students in mainstream schools" where deaf students "are taught in their resource classroom" (Smith 2003: 43); itinerant teacher of the deaf service, through which these teachers "support deaf students who are mainstreamed individually in their mainstream schools" (Smith 2003: 44); and resource services for deaf students, which include "educational and technical support services [offered] to all deaf and hearing impaired students in their region" (Smith 2003: 44). The Ministry of Education provides a national advisor on deaf children service "to preschool children and their families and to students educated in mainstream placements" (Smith 2003: 44). Additionally, teacher aides and part time teachers may be provided under Ministry of Education support services, who are "employed to support [deaf] students in classrooms and give individual instruction" (Smith 2003: 44). Consistent with international trends (Antia et al 2010, Sheridan 2008), more and more deaf children are being placed in mainstream education services in New Zealand, especially given the growing prevalence of cochlear implants and other technology.

Decisions about education play an important part in the lived experiences of the participants in this study, and are influenced by decisions about cochlear implants. In this respect, decisions about education follow on from the decisions about $\mathrm{Cls}$ and communication that focus on participation and inclusion in the mainstream, hearing world. All of the cochlear implant users in this study attended mainstream schools, and most did so for the duration of their education; in choosing a cochlear implant for their children, this tended to be the 
intention of the parents in this study. Hannah attended Kelston Deaf Education Centre for preschool and her first school years, and then moved to a deaf unit within a mainstream school, before being fully mainstreamed. John noted their intention for Hannah to transition to mainstream education: "Yeah, no the plan was to get her mainstreamed, otherwise there was no point having an implant, you know." With the goal of good access to spoken language, and involvement in mainstream society, many cochlear implant users are not exposed to sign language during their school years. The participants in this study were successful throughout their school years, and all achieved highly in mainstream schools, which, undoubtedly, would have reinforced parents' decisions to continue to pursue mainstream education for their children.

Sandra considered moving Adam to van Asch in Christchurch, but felt that it was a better environment for her and Adam in their hometown, especially with access to family support. Furthermore, on considering anything other than mainstream schooling for Adam, Sandra explained that this occurred

about the time I had to make the decision about whether he'd be signing or not. I thought, and I think somebody said to me out of audiology or somewhere along the way, and said, 'look, really by seven if they haven't grasped language, they're going to battle after that, they're just getting beyond that point that, you know, if they were struggling with it, spoken and written and hearing side, that, you know, they might be better to communicate sign wise.' So I had told myself to that point to decide that if he really battled, I would just make a decision to take him to one of the schools, despite not liking van Asch, but if that was the best thing for Adam then that's what I would have done. But he didn't, he really did surge, he just did so well, you know, and I just felt that he was fine, [...] you know, he would always take priority, but he really did do so well, so at that stage I decided to stay in a mainstream school.

Sandra's reflection on this process of decision-making illustrates that, whilst deaf education is presented as a choice, it tends to instead be seen as a fall-back option, which demonstrates the dominance of the mainstream model. Thus, deaf education is seen to be 
inferior to mainstream education, and choosing deaf education is perhaps seen as reinforcing that deaf children are different and do not fit in with wider society (cf. Kent 2003: 316).

Drawing on her observations and experiences as a teacher of the deaf, Olivia discussed this theme of deaf education as a fall-back option.

And that's true in Australia as well, so what you're seeing is parents who make a decision, for whatever reason, that their kid's going to be mainstream [...]. But then the gap continues to widen and you either start seeing behaviour problems because they're frustrated and they can't do what's being asked of them, or there's a real academic concern because whatever the mainstream teacher is doing isn't working for that kid, so that you start seeing them transition at sort of intermediate age into provisions, and I think it's because parents and teachers get concerned about this gap that gets bigger and bigger, as far as language and if that language gap continues to widen then there are social implications, and there are emotional implications, and there are academic implications. So I think thematically, while I've never said, 'well why are you considering sending your kid to my provision?' I do parent tours all the time, [and] very thematically what's being said is, 'what we're doing now isn't working so we've got to consider a more specialist option.'

As a teacher with a wider overview, Olivia highlights the variability of outcomes for cochlear implant users, and observes that using a $\mathrm{Cl}$ does not necessarily equate to automatic inclusion in the hearing world of school. As the next chapter will explore, $\mathrm{Cl}$ users do not necessarily experience an easy or straightforward inclusion in the hearing world. In this sense, while parents may perceive that their children are doing well in the mainstream and are well integrated into the hearing world, this is not necessarily how deaf people with Cls feel about their lives. Although some $\mathrm{Cl}$ users 'succeed' in terms of acquiring speech, and can 'pass' in the hearing world, others struggle with this access and, further still, all $\mathrm{Cl}$ users will encounter some situations to which the technology is not able to facilitate access to. 


\section{CONCLUSION}

The decisions about cochlear implants, along with consequent decisions about communication and education, demonstrate the power of normality and the desire to be included in the mainstream. From the birth of a deaf child, hearing parents are likely to become enmeshed in a medicalised trajectory of decision making, in which the impairment of deafness, and attempts to alleviate it, becomes central. The decisions made by the parents in this study demonstrate the influence of social and medical norms, but also reflect the hopes and expectations that these parents held for their children. In deciding on cochlear implants, these parents hoped that their child would be able to live as 'normal' a life as possible, and be a part of their own hearing culture. For the $\mathrm{Cl}$ users who made the decisions about implants themselves, such decisions were largely informed by the possibility of furthering their life trajectories within the hearing world, and therefore not losing contact with already established social groups. Furthermore, the retrospective narratives of my participants justify their decisions as 'good' decisions, and thus situate them as part of the reflexive project of creating coherent and positive life narratives (Giddens 1991). 


\section{CHAPTER FOUR: NEGOTIATING LIFE WITH A COCHLEAR IMPLANT - THE EMBODIMENT OF TECHNOLOGY}

\section{INTRODUCTION}

What are the everyday realities of being deaf and having a cochlear implant? In this chapter, I draw on participants' narratives to explore the practical aspects of cochlear implant technology. I use descriptions from the cochlear implant users, parents, and the teacher of the deaf, in the hope of developing a picture of the embodied, lived experiences of my participants. Here, I demonstrate that Cls are not a 'cure' or a 'quick-fix' for deafness, and that cochlear implant users still face a number of obstacles in the hearing world. It is important to note that all of the participants in this study spoke positively of the impact that Cls have had on them, and could have on the lives of deaf individuals and their families. However, this study's sample is by no means representative of the experiences of wider groups of cochlear implant users. Whilst none of the participants in this study were negative about their implants, it became clear that there are certain everyday hassles to contend with, issues with awareness of deafness and $\mathrm{Cls}$, and an acknowledgement that a $\mathrm{Cl}$ does not make a deaf person hearing. I explore the personal, bodily, social and cultural effects of cochlear implants, and employ Margaret Lock's theme of 'local biologies' (as discussed in Chapter Two) in order to understand the embodied experiences of this group of $\mathrm{Cl}$ users.

Little research has been undertaken on the real, lived experiences of having a $\mathrm{Cl}$, and, furthermore, little has focused on the voices of $\mathrm{Cl}$ users themselves. Preisler et al (2005) draw on Blume (2002), who "found that in studies showing positive effects of cochlear implants in children, the children themselves were never asked their opinion. Opinions on the effects were provided either by experts, such as surgeons or researchers, or by parents in response to questions about their child's well-being" (Preisler et al 2005: 261). The study by Preisler et al (2005), in which eleven $\mathrm{Cl}$ users were interviewed, addresses this gap, and covers some of the issues raised in this chapter. However, these $\mathrm{Cl}$ users were aged between eight and ten years old; I believe that interviewing older $\mathrm{Cl}$ users was beneficial in regards to their sophisticated reflections on their experiences with deafness and the technology. I 
follow Best et al in their conceptualisation of technology, in that it consists of "networked material objects, systems of knowledge, conventional practices, and the meanings with which they are imbued, together creating apparently coherent entities which articulate with the contexts in which they are embedded" (2013: 110).

\section{The TECHNicAl Fragility of Cochlear Implants}

The experiences of my participants indicated that $\mathrm{Cl}$ technology is not practical at all times. Cochlear implants are incompatible with certain situations - contact sport and some outdoor play, for example; are unusable in some circumstances - in water, for example; and cause discomfort for $\mathrm{Cl}$ users on occasion - around static electricity, for instance. Whilst this can be frustrating at times, Hannah, Julie and John in particular recalled some situations in good humour.

Julie: [At a beach with] Iron sand. She rolled down the beach, iron sand, we never thought anything of it [laughs], taking [her] blonde hair up and it was all this black pile of sand stuck to her implant, we couldn't clean that out for love nor money, could we?

Hannah: No we just had to replace it.

This quote illustrates that cochlear implant technology, for Hannah, has become embodied in the self, and entangled with bodily, subjective experiences (Csordas 1984: 5). However, once the Cl's use is inhibited in some way, and therefore constrains the body to an extent, the technology becomes "insistently present" (Messinger 2010: 291). In this respect, Cl users must adapt to the shortcomings of the technology and incorporate knowledge of these limitations into their bodily routines and actions.

As a piece of technology, cochlear implants are subject to technical limitations, glitches, and breakages. Thus, a $\mathrm{Cl}$ may give someone the potential to hear, but it does not take away someone's deafness. In this study, Hannah explained, "so you have a cochlear implant from when you are one, but you're still deaf - do you shower with it on? Do you go swimming? Do you sleep with it? No, you can't. The batteries die, you rely on batteries." Adam recalled 
how, when he was around 16 years old, "my cochlear implant just randomly broke down and I [didn't] have any hearing for about a week or so, and I had to [...] come to school and attend classes, but I ended up reading a lot of the note takers' notes." Furthermore, Julie recalled the difficulties they encountered when Hannah had an accident and her $\mathrm{Cl}$ was damaged:

But because when she had her accident, her implant came off and she had a head injury, and so no one was going to put the implant on her again, because they didn't know what the head injury was at that [stage] [...] so we were in hospital with someone that was oral and all of a sudden that had been taken away and we were back to a deaf person, [laughs] and they're trying to say things to me like, 'can you sign to her,' [...] and [...] we didn't have, we'd lost it [sign language], and that was a big wakeup call too.

These narratives demonstrate that, despite much dependence on $\mathrm{Cl}$ technology by both users and their families, especially in regards to communication, cochlear implants are still a fallible, fragile technology. This leads to the vulnerability of $\mathrm{Cl}$ users when they encounter situations in which they are unable to use their implant and, given the focus on 'mainstreaming' deaf children, along with the emphasis placed on spoken language, these individuals often lack an alternative in such situations. Furthermore, these narratives highlight that these $\mathrm{Cl}$ users are physically both deaf and not deaf in some respects and in different contexts, and that their social selves have to assimilate both of these embodied realities.

All of the $\mathrm{Cl}$ users in this study grew up predominantly using spoken language, but, if their $\mathrm{Cl}$ was not working, participants had to use alternative forms of communication. David recounted how when his son Connor wakes up, "I have to sign, or I have to [...] write a note, because there's no other way I can actually communicate what's going on." Thus before Connor has his cochlears working, and, “because we don't do fluent sign language, there's a bit of an impasse, and as soon as it's anything complicated, like trying to introduce you [Hayley], you have to write it all out." For Adam and his mother, basic sign bridges the morning period before using his implant. 
Adam: [My mum] knows a little bit of basic sign language. For example, if I wake up in the morning before school, she always woke me up with the sign, 'school time' [...]. So it was just like a basic sign that helps communicate with me before I put my cochlear implant on in the mornings. 'Cause like sometimes I get lazy and I don't really want to have to listen so early in the morning, I like put it on midmorning. So she kinda just signs to make it easier.

This importance of an alternative means of communication further demonstrates the limits of $\mathrm{Cl}$ technology and the strategies that users must develop to cope with such limitations. Such strategies demonstrate the ways in which Cls have been incorporated into family life and the everyday coping strategies that ensue. These examples also show, however, that the importance placed on hearing and spoken language may inhibit the development of alternative communication, which is necessary given the fragile nature of $\mathrm{Cls}$. In this respect, while families may maintain basic sign language, the focus on spoken language often means that any conversation that requires more than simple signs is unachievable without the deaf individual using their implant, which deepens dependence on the technology.

\section{'IT's SO HARD THIS HEARING THING'}

The effort that $\mathrm{Cl}$ users need to put into hearing is often overlooked in mainstream society, especially given widespread misconceptions that $\mathrm{Cls}$ enable deaf people to hear as hearing people do. Participants commonly talked about how tiring hearing can be for $\mathrm{Cl}$ users and how, sometimes, they need a break from the intensity of constant sound.

Jack: [...] because it's hard for the parents to understand that because they're not wearing it, because I've spoken to some adults and they're like, 'yeah I don't like to put my cochlear on in the morning, I just like to have the peace,' I'm like, 'yeah same!' And then I hear parents go, 'Bob doesn't like to wear his cochlear implant in the mornings - what's up with that? The doctor said I should like force it on him.' And I was like, 'what?! Are you kidding? It's so hard this hearing thing!' 
Furthermore, Julie, the mother of $\mathrm{Cl}$ user Hannah, noted, "I do forget sometimes [...] the amount of concentration Hannah has to have to listen, and how tiring that is." On this, Sandra said of her son Adam's experiences, "He's always battled to listen, always gets really tired and struggles." Additionally, drawing on her experiences teaching children with implants, Olivia stated, "their batteries will go flat and they'll never tell anyone because they just enjoy the [quiet], because it's loud, it's a lot of stimulation [...]." It is clear that the physical experiences of hearing can be overwhelming and demanding for the $\mathrm{Cl}$ users in this study, and that, despite their use of this technology, the hearing world is not always predisposed to their needs as deaf people. However, these difficulties become embodied in the deaf body, with these $\mathrm{Cl}$ users constantly concentrating on, and feeling tired from, hearing and interpreting sound. In this sense, $\mathrm{Cl}$ users' 'local biologies' (Lock 2001) reflect the entanglement of technology and its limits, physical bodily and cognitive experiences of sound and language, family practices, and social expectations surrounding hearing.

The $\mathrm{Cl}$ users in this study spoke positively about the effects that their $\mathrm{Cls}$ have had on their lives, and of what this technology has enabled them to be involved in and achieve. However, there are some sounds and types of hearing that $\mathrm{Cl}$ users tend not to have access to. In particular, directional hearing poses issues for $\mathrm{Cl}$ users, and they are often unable to establish where sound is coming from.

Hannah: I don't have any directional hearing - you know, I could be walking down the street and I'll hear sirens, I'll be like looking around going, 'where are the sirens coming from?' Someone will be like, 'it's coming from that direction,' I'll be like, 'oh ok.' I can't hear it. And one thing my mum does - its drives me absolutely up the wall. She raised me, you know, a deaf child! And she knows I can't hear directional hearing - if I come home, go, 'mum where are you?' She always says, 'I'm here.' I'm like, 'where?' She goes, 'oh I'm in the lounge sorry [...]'.

This lack of directional hearing illustrates that Cls do not provide natural or complete hearing. Anna and David, however, noted that Connor's second implant has aided in his access to directional hearing. David recalled that "when he only had one we'd be calling him somewhere in the house and he wouldn't have a clue where the sound was coming from 
[...]. With two, it immediately enabled him to be able to hone in on where's this sound coming from, that way or that way."

Background noise proves to be a hindrance to hearing and communication for $\mathrm{Cl}$ users. Within private family homes, some adjustments have had to be made to cater to $\mathrm{Cl}$ users, making the home suitable for their deafness. Julie recalled that there were no captions on television when Hannah was growing up, which meant that Hannah was unable to understand it. Accordingly, she noted, "you'll notice that's another thing that we find, because growing up with Hannah we had a very quiet house, [...] and we did that on purpose because any background music used to take [Hannah] away from us." Anna and David spoke about adjusting to being unable to have music playing, with David noting, "I can't play my music, because even if I have it down low [Connor] will just go and turn it right down. He just doesn't like it." Isaac also spoke about adjustments within his home to accommodate his needs: "there's a couple of small things that you'll notice around my house, like being very well lit, that kind of thing, which is good for lipreading [...]."

Noisy public places can also pose a problem for $\mathrm{Cl}$ users. David expressed how, as parents of a deaf child with cochlear implants, it is vital to be aware of the environments that they enter into with Connor.

[P]art of us being parents and being aware of deafness is being very aware of going out in socialised places, socialising cafes, restaurants. And $[\ldots]$ as a result being extremely aware that Connor is really not able to socialise $[\ldots]$. But there are a number of places where we've been for dinner - just in ordinary restaurants or cafes - and we suddenly become very aware of those places, a lot of venues, that are [...] so live, there's no dampening of the noise, there's no curtains, there's no carpet, so you've got wooden floors and brick walls, and when you've got a lot of people in there it's almost, I mean I know I find it hard to hear what people are saying, well for Connor it's completely...I mean to cope with that, and I think that's something else that we became extremely aware of. 
Correspondingly, Hannah spoke of how she has no control over what sounds her $\mathrm{Cl}$ picks up, and the frustration that this causes her.

If you go to a shopping mall and you try to talk to me, I'll hear the background, well not the background but the 'meeeehhhh' noise. But I can pick up little bits of what you're saying. [H]earing people, they've got the skill of cancelling out background noise and focusing on what they want to hear, I don't have that. The machine does it for me, so if it decides the motorway is what I want to hear, it'll cut you out and listen to the motorway, [...]. Yeah I don't have any control over the machine. So, oh not the machine, cochlear implant - really shouldn't say the machine but that's what, that's what it is.

Hannah's narrative highlights her reflexive understanding of the machine nature of her $\mathrm{Cl}$ and, as noted above, the insistent presentness of it when its limitations are exposed. In the example that Hannah uses above, she makes it clear that she is aware of how her Cl's limits intrude on her and her experiences, and that her body has not seamlessly incorporated the technology, nor gained control over it. Marx refers to tools in a similar way; they feel like extensions of the self when they are working, but when they break (or need new batteries in the case of $\mathrm{Cls}$ ), we become aware of them as objects separate from us, and as fallible objects made by people (Marx 1887: 127). Thus, the embodiment or seamless incorporation of a $\mathrm{Cl}$ is disrupted by the limitations of the technology.

\section{THE Social WorLdS of Cochlear IMPLANT Users}

As described in the quote from David above, socialisation can be difficult for $\mathrm{Cl}$ users in noisy or group environments. In reference to interaction in groups, Jack stated that, "it's still helpful if there's only one person talking, but with a cochlear it's like, you can get up to about three people talking and you're still coping, but anything more than that, it's like, 'oh man, what's going on here?"' Adam and Isaac both spoke about how difficult group situations can be in terms of following what others are saying. Adam noted, "I sit alone [in the dining room at his university hostel] because it's easier for me. I don't have to talk to people, I don't have to be like, 'What? What?' Annoy everyone. I just read and eat at the same time, it's easier." Here, Adam's awareness of the limitations of his implant in group 51 
situations have resulted in him isolating himself from others in these circumstances. Isaac noted similar sentiments:

It's still hard now [in group situations] [laughs]. So I do tend to avoid group situations as much as possible. But yeah when it happens, it happens, and I get into it - I either switch off or ignore other people and make my own conversation and go from there. It's a lot easier to listen to what other people are saying if you know roughly what they're going to say. [...] yeah if I just walk into a conversation it takes me a long time to pick up what's being said, and what the topic is, etcetera. Whereas if I go up to someone and start a new conversation, I can follow it a lot easier.

Therefore, while the goal of $\mathrm{Cl}$ implantation is often to improve social integration and interaction within mainstream society, the limits of the technology sometimes result in forms of social isolation. This isolation was especially evident when participants did not have connections with other deaf children or others with cochlear implants, as was the case for most of the $\mathrm{Cl}$ users in this study throughout their childhoods. So, whilst $\mathrm{Cls}$ partially connect and integrate users into hearing social worlds, their use can also act to isolate them from d/Deaf social worlds.

However, some of the participants in this study described improved inclusion in conversation and group situations once they were implanted. This was the case for Isaac and Jack, who were implanted when they were older. On this, Jack stated, "Socially, yeah it's [...] definitely a confidence booster, being able to go into a situation, like know that you have a fairly good chance of understanding the conversation." Furthermore, Anna and David also spoke of differences in their son Connor's access to social, communicative situations after he had received his second $\mathrm{Cl}$.

David: [...] But I suppose there's also another aspect which relates more generally to the cochlear, to the deafness area, is how they miss out on the nuances of social interaction, which in the early years, that is certainly something that he's [Connor] had to do a lot of catch up with. [...] I remember his second cochlear enabled him to hear a lot 
more of what was going on, because what was happening in his peer group, stuff happens so fast and people say something and suddenly they're off doing something else. Quite often he would miss that, and so he wouldn't understand what's going on - I think I remember him saying the second cochlear enabled him to be more aware of what his mates were talking about and doing. So that, that obviously really helped from that point of view, socialising.

Hayley: So you think it helped socially?

Isaac: Yeah just because you can pick up what people are saying, you can insert yourselves into conversations more easily and make friends that way, whereas before I can't do that. [...] So yeah, it gave me a bit more of a confidence boost in being able to hear other people, being able to follow what other people were saying. Still wasn't perfect but it was something.

Again, it is evident that $\mathrm{Cls}$ are perceived differently by their users depending on their effect in different circumstances. While these narratives exemplify that $\mathrm{Cls}$ do not make their users physically hearing, my participants noted the increased confidence and self-esteem associated with the ability to be able to interact in more social situations.

\section{'I CAN’t Quite IMagine Life Without IT'}

Despite the issues raised in this chapter regarding the limitations and everyday practicalities of cochlear implants, all of the $\mathrm{Cl}$ users, as well as their parents, were positive about their overall experiences with their $\mathrm{Cls}$, and were grateful for the opportunities the technology had given them access to. Hannah stated, "Obviously the positive [of a cochlear implant] is you can use it to your advantage - you've actually got the choice, wake up one day, I wanna hear, wake up the next day, I don't wanna hear." Hannah's assertion here links in to the themes discussed in the following chapter, in that she sees her implant as giving her choices. Isaac also spoke positively of his $\mathrm{Cl}$, noting, "it's definitely made a huge impact, and I can't quite imagine life without it - that would be really weird." Here, Isaac furthers the notion of 53 
$\mathrm{Cl}$ users embodying the technology, where, when it is working, it becomes an extension of their bodies and selves. Additionally, Jack, who received his implant when he was 18, reflected on his $\mathrm{Cl}$ and the influence that it has had on his life:

I understand that having a cochlear will grant me a better chance in jobs and relationships, but at the same time I'm not ashamed of being deaf, it's like I got this [cochlear implant] so then I could continue on the way I was growing up, but I also have to accept that I'm deaf, but it's cool [...].

Jack's narrative highlights the constant presence of multiple facets in identities, and our multiple selves or identity constructs (Leigh et al 1998). Here, he is indicating that his identity incorporates aspects of his involvement in the hearing world, as well as his deafness. For him, it is important to maintain the connections in the hearing world that he grew up with, but also to recognise and be accepting of his deafness. His identity work thus involves expressing a flexible, hybrid identity, in which he incorporates and acknowledges aspects of both $d /$ Deaf and hearing cultures in his sense of self (Smith 2008).

\section{(MIS) UNDERSTANDINGS OF COCHLEAR IMPLANTS AND DEAFNESS IN}

\section{MAINSTREAM SOCIETY}

Perceptions of deafness in the hearing world are dominated by medicalised views of normalcy. Treating deafness as something that needs to be 'fixed' or subjected to medical care underlies common misunderstandings of the lived experience of $d /$ Deafness. Jack expressed that "a hearing person wouldn't know what it's like to be deaf, but they'd probably see it as a negative thing due to the mass media and doctors." This lack of understanding might also be compounded by the fact that the majority of deaf children are born to hearing parents. Jack articulated that "there will be that lack of understanding of how a kid might be feeling, which you know is, it's something that's hard for a hearing parent to understand who didn't embrace their child's culture almost, because they're almost born into a culture, if you're born deaf." In this respect, $d$ /Deaf people, like disabled individuals, "differ from members of other stigmatized groups in that they were not reared by disabled parents, nor did they grow up among disabled people" (Murphy et al 1988: 241). 
Consequently, most $d$ /Deaf individuals do not receive any socialisation into d/Deaf worlds through their families, who may not understand the social issues $d$ /Deaf people face, or the centrality of deafness in the lives of these individuals. Thus, kinship is sometimes "the sphere in which the family has to confront ways of disciplining and containing [...] stigma" (Das and Addlakha 2007: 128-9). This shows us that local biologies of "disability and impairment [are] located not in (or only in) individual bodies, but rather [...] within a network of social and kin relationships" (Das and Addlakha 2007: 128-9).

From an educational perspective, Olivia stated that the deaf population is "generally [a] misunderstood population because people [...] say, 'well why don't they just read?' These people don't ever think about what language is and what it means to have had access to language since you were born, really since you were in utero." This misunderstanding, and common misrepresentation, of deafness within wider society ties into the theme of liminality, in that $d /$ Deaf individuals are often perceived to be outside of the norm, and therefore marginal to mainstream society. In this respect, $d /$ Deaf people may occupy a liminal 'state' as they are "caught and fixated in a passage through life that has left them socially ambivalent and ill-defined" (Murphy et al 1988: 235).

The participants in this study regularly experienced instances in which people were unfamiliar with cochlear implants. Most of the participants noted, sometimes in frustration, that they are often asked about their implants. On this, Isaac said, "people look at it and think, 'what is it?' And I tell them it's a hearing aid but bigger, and say cochlear implant, and they're like, 'cool.'" Furthermore, on experiences after receiving his implant, Jack stated,

[I]t's probably also to do with people's awareness of the abilities of cochlear implants too, because I guess some people are like, 'oh you've got a cochlear, you've got good hearing,' but it's like, 'oh almost, no, sometimes a little bit harder.' 'Cause I guess some people's awareness of deafness is still lacking I guess.

As Jack notes, if people are aware of cochlear implants, they often have a skewed idea of what the technology can do. In this sense, medicalised views of Cls, as well as the media's presentation of them as 'miracle cures' (Blume 1997, Edwards 2005), contribute to 55 
sometimes unrealistic ideas of what implants can actually do. Thus, as Jack asserted, it can be difficult to explain how a $\mathrm{Cl}$ user actually experiences sound and speech. These misunderstandings, in turn, can lead to uneasy experiences in the hearing world, which may contribute to a sense of marginality or liminality.

\section{CONCLUSION}

Participants' narratives asserted the importance of the material, physical and embodied experience of both $\mathrm{Cls}$ and deafness to their senses of self. They made it clear throughout interviews that Cls are not a replacement, or a substitute, for 'normal' hearing, and thus in their narratives challenged medical and mainstream understandings of this technology. Part of their identity work therefore involved coming to terms with the limits of the technology and its promise to transformatively eliminate their deafness. In this sense, these $\mathrm{Cl}$ users see themselves as still at the threshold of the hearing world, as their Cls do not enable them to fit into the hearing norms of mainstream society, nor fully involve themselves in hearing social worlds. On this, Adam noted, "a cochlear implant helps me [to] hear, but it's not [a] substitute for normal hearing, because it's not perfect." Hannah, in talking about the prevalence of unawareness of both deafness and cochlear implants amongst hearing people, stated, "I can hear - but I don't hear as well as hearing people." In narratively emphasising this lack of social awareness about their bodies, experiences and social challenges, $\mathrm{Cl}$ users were demonstrating the social distance that sometimes existed between themselves and hearing people. However, participants' narratives, like their identities, are complex. They also emphasised the value of expanded choice and social opportunity within the hearing world, a topic to which I now turn in Chapter Five. 


\section{CHAPTER FIVE: NARRATIVES OF "CHOICE”: \\ PROBLEMATISING THE IDEAL}

\section{INTRODUCTION}

What role does the cultural value of choice play in the decisions about cochlear implants? The participants in this study used narratives of choice throughout interviews, emphasising the opportunities that choices might facilitate. This chapter reflects upon participants' ideas that having a cochlear implant has given the $\mathrm{Cl}$ users choices, and the ways in which they construct their identities and hoped-for life trajectories through the idiom of choice. Thus, participants saw these "choices" as tied closely to the role that a $\mathrm{Cl}$ might play in access to desired opportunities. Such opportunities tended to specifically refer to language use, social identity and networks, and academic achievement. Whilst this section draws largely on the narratives of parents, particularly in the form of their hopes for their children, it is clear that, in some respects, the $\mathrm{Cl}$ users reflect similar narrative themes and ideas. It is important to note that the opinions from parents here tend to be referenced in terms of the hopes that they held for their children when they decided on a cochlear implant. However, they did also speak about the fulfilment of these hopes, and the success of their children, which, in part, was attributed to the choices and opportunities that an implant had given their children access to. Correspondingly, the $\mathrm{Cl}$ users expressed how having an implant had allowed them to make their own choices as they grew up, and had facilitated access to certain opportunities. It is important, however, to interrogate ideas of "choice," and to explore the social factors that shape choices. This chapter, then, further illustrates the power of hearing norms and the expectations of medical and mainstream paradigms in dealing with deafness.

\section{Theorising Choice and its Value in Modernity}

Choice, and the ability to exercise it, is a key value of modern identity (Rapp 1999, Sointu 2005, Mol 2008), exemplified by the emphasis that both the parents and the $\mathrm{Cl}$ users in this study place on the concept. Their references to the concept of choice were evident in their decisions about cochlear implants, education and language, but, as is primarily drawn out in 57 
this chapter, also in their narratives about the choices that these decisions have enabled. Rayna Rapp characterises choice as a central "cultural value and strategy" (1999: 138) in Western settings, and notes "the subtle, perhaps fetishizing aspects of individualism implied in the concept of choice" (1999: 226). Furthermore, she asserts that technology has transformed choice on an individual level (Rapp 1999: 140). In that "the ability to exercise choice [is] turning into the key value of our times" (Sointu 2005: 263), Sointu suggests "exercising choice indicates that one is a full and 'normal' subject. As such, the contemporary individual is arguably "not merely "free to choose", but obliged to be free" (2005: 263). In their beliefs that implants may enable particular choices in the life courses of $\mathrm{Cl}$ users, my participants also suggest that choice was a core value and narrative frame for understanding the events and successes of their life trajectories.

Anthony Giddens (1991) explores choice and its place within society in the late modern age, and states that, in modern daily life, individuals are increasingly "forced to negotiate lifestyle choices among a diversity of options" (1991: 5). In this respect, "lifestyle choice is increasingly important in the constitution of self-identity and daily activity" (Giddens 1991: 5). Furthermore, he states,

On the level of the self, a fundamental component of day-to-day activity is simply that of choice. Obviously, no culture eliminates choice altogether in day-to-day affairs, and all traditions are effectively choices among an indefinite range of possible behaviour patterns. Yet [...] modernity confronts the individual with a complex diversity of choices and, because it is non-foundational, at the same time offers little help as to which options should be selected (Giddens 1991: 80)

The "multiple possibilities" (Giddens 1991: 84) of choices contributes to the complex and ongoing decisions involved in the formation of self-identity. In the context of this study, the narratives of participants exemplify the importance attributed to navigating choice and the availability of multiple possibilities.

However, despite our idealisation of choice in modernity, Annemarie Mol (2008) problematises choice and its role in wider society. Within her work on patient choice in 
health care, Mol acknowledges that "[i]ndividual choice is not only celebrated as an ideal in health care," it "surfaces everywhere" (2008: 3). Mol states that, despite beliefs that the "difference between autonomy and heteronomy has [...] come to mark the difference between 'the West' and 'the Others'" (Mol 2008: 3), individuals in the West are not autonomous individuals (2008: 4). In this respect, although we indeed have choices, these choices are not independent of external influences. Furthermore, these choices are contextual and reflective of wider social discourses. Therefore, while the individuals with $\mathrm{Cls}$ in this study may have access to a wider range of choices and opportunities because of their implants, these choices are influenced by social norms and possibilities, technological developments, and their place within social networks. Furthermore, their own choices are tempered by the decisions and choices that their parents, and medical and educational professionals have made for them. Additionally, while this ideal "certainly infuses many practices, the logic of choice does not inform everything that happens in 'the West'" (Mol 2008: 91). Consequently, the $\mathrm{Cl}$ users must manage the ideal of choice along with other ideals, such as independence, individualism, and success. Lastly, the ideal of choice may be interpreted differently by different people. Thus, the parents in this study, for example, may see choices in a different way to their children. Moreover, the decisions and choices that they have made for their children, whilst possibly enabling further choices, are not always reversible; they can limit choice in the lives of others. In this respect, in practice, available and appropriate choices can be heavily circumscribed (Best et al 2013: 117).

Throughout the majority of media rhetoric on cochlear implants, the technology is often presented as a "miracle" or a 'cure' for deafness (Lane et al 1996, Edwards 2005). Within the New Zealand media, articles on cochlear implants tend to focus on the opening up of, and new access to, the world of sound, and the transformative benefits of $\mathrm{Cls}$ (Bathard 2012; for examples, see O'Neil 2012, Torrie 2012, Penman 2012, Shadwell 2012). Simultaneously, deafness tends to be represented negatively, "with particular emphasis placed on silence, parents' fears for their deaf children, and worries over deaf children falling behind at school" (Bathard 2012: 29). Despite medical and public rhetoric about cochlear implants, the parents in this study, far from believing that an implant would make their child hearing, spoke instead of the choices and opportunities that an implant would give their children. It is important to note that, whatever decisions and choices these parents made, they were clear that they were doing what they thought was best for their children and their family unit. 
Generally, these parents believed that a $\mathrm{Cl}$ would give their children choices in terms of being part of hearing or $d /$ Deaf worlds, and that these choices would give them access to a wider range of opportunities throughout their lives. Given that a $\mathrm{Cl}$ might improve the hearing of their children, and therefore their speech, these parents viewed cochlear implants as a tool which might give their children more, and perhaps easier, access to people, wider society, education, and the workforce. In a study with deaf mothers who had chosen cochlear implants for their children (Mitchiner and Sass-Lehrer 2011), participants reflected on "choice" as a central reason behind their decisions. The mothers in Mitchiner and Sass-Lehrer's study asserted that their reasons for choosing Cls "were strongly related to their desires to provide their children with more communication and language choices. Even though they had a range of different reasons, they repeatedly emphasized giving their children more choices in life and the ability to communicate with more people" (2011: 80). I argue, however, that whilst there is no doubt that a $\mathrm{Cl}$ may give its user options that may not have been open to them otherwise, it is important to question the meanings of choice, and to recognise that all choices are mediated by social and cultural settings, and circumscribed by social norms.

\section{'Give Her a Choice, Give Her a Chance'? Exploring Notions of "Choice" Through Cochlear Implants}

For Sandra, whose son Adam was implanted when he was three years old, choices and opportunities surrounding language were central in her decision about a $\mathrm{Cl}$. She was very clear about her wishes for Adam when she was choosing a $\mathrm{Cl}$, especially in her desire for him to have access to both spoken English and NZSL. Despite encountering some opposition to her decision about using both spoken and signed languages, Sandra was firm in her belief that she was doing what was best for Adam.

But when it's your own child and you're thinking about options, you're going to think about the best, the way to give them the most options growing up. In my mind I couldn't see the problem that he had a cochlear and signed, in New Zealand Sign - I thought if he had both options, he's covering all bases, and he can choose when he's old enough to make a decision if he wanted to take the cochlear off, get rid 
of it, and, you know, as long as he maintained his sign and his cultural side, as long as he got access to that, I couldn't see an issue at all.

Furthermore, Sandra stated, "if it was your child, if you took up this cochlear/hearing debate, you want every opportunity for them, to make their way, you do everything you can, and then they decide what they need. If I took one option away, I felt that [...] I wasn't really giving him both opportunities." Sandra's narrative here demonstrates that one of the roles she sees as being important as a parent is to enable Adam to be empowered in his own choices, and to have the ability to make these choices as an independent, individualised agent who is self-responsible. These notions reflect ideals of parenthood, in which parents have high aspirations for success, independence and self-sufficiency for their children (Kent 2003). Deafness can represent a threat to such aspirations for hearing parents, heightening their desire to give their children access to more options and choices.

As Sandra's above quote demonstrates, she hoped that Adam would use both spoken English and NZSL, which, in turn, would facilitate his engagement with both $d /$ Deaf and hearing worlds. However, given the lack of support that Sandra and Adam received for NZSL, along with the emphasis placed on speech acquisition for $\mathrm{Cl}$ users, Adam's access to NZSL was cut short when he was at primary school, as discussed in Chapter Three. Thus, within such a dominant medical paradigm, choices may appear to be 'free' and unrestrained, but are in fact limited. Such restrictions are also furthered by the general lack of availability of NZSL, despite its status as the third official language of New Zealand (Fitzgerald et al 2013, Best et al 2013). Additionally, Adam had little access to the $d /$ Deaf world and other deaf people, as there were few socialisation opportunities available in their hometown. This further demonstrates that, despite Sandra's hopes for Adam to be engaged in both $d /$ Deaf and hearing social worlds, his choice to do so is constrained by external factors, such as the small size of Deaf communities in New Zealand (especially outside of main centres), and the lack of information provided to parents, within the medical system, on Deaf culture and communities (McKee 2006, Sawicki 2008, Best et al 2013).

Julie and John, like Sandra, were of the belief that a cochlear implant would give their daughter Hannah more choices as she grew up. For them, though, having an implant was 
also tied to success in the wider hearing world, given the possibility that the technology would facilitate easier access to sound and speech. Julie and John hoped that Hannah would be able to participate fully in wider society, and they believed that having a $\mathrm{Cl}$ might aid in this involvement.

John: At the end of the day, I mean our choice with Hannah to have the implant is that, you know, quite callous, we wanted her to be a tax payer, not a tax taker, right, and do the best that she could. Give her a choice, give her a chance, you know, don't hold her back. I mean, if it didn't work, it didn't work, but I mean, you don't know that at the beginning.

John's reflection on the hope that a $\mathrm{Cl}$ might help enable Hannah to be a tax payer reveals more implicit ideas of successful individuals in contemporary New Zealand society. Within Western cultural expectations, 'acceptable,' 'successful' individuals should "compete for success, conform to expected standards, and are independent, self-sufficient, and popular" (Kent 2003: 316). As John referred specifically to paying tax, notions of a successful individual being a member of the workforce, and thus a contributing member of society are also elicited. In this sense, as Jackson asserts, "[w]ork produces both self and societies [...]. Not only does work provide the livelihood of persons, it creates modes of sociality and sustains a vital sense of what it means to coexist and cooperate with others. Accordingly, human labour [...] is the means whereby human beings create and recreate the intersubjective experience that defines their primary sense of who they are" (1998: 16). Lane et al (1996), in a North American context, argue that many parents of deaf children worry that their child will not be a productive member of society, which, in turn, challenges the centrality of work in contemporary life. Thus, for parents, cochlear implants may provide a way in which their children can participate in society, overcome their 'difference,' and become a 'contributing' member of wider society.

Furthering the narrative of choice in these parents' accounts, Sandra was clear in her belief that her choice to give Adam an implant, at the least, would enable him to make the ultimate decision about his cochlear when he was older. Similarly, Julie and John were explicit in their beliefs that deciding on an implant for their daughter Hannah would enable her to choose her own path in the future. Like Sandra, they spoke of this choice in terms of 
the ultimate decision being in the hands of the deaf individual. It is widely acknowledged that late implantation is not as successful as early implantation, especially in terms of access to spoken language, so the narratives of these parents illustrate that, rather than wait until their child was older to make the decision, they made the decision on the proviso that their child may later choose not to utilise the technology. Although these parents believed that their decisions on Cls meant that they weren't taking away any options from their children, it is important to recognise the pervasive value placed on being able to hear and speak. Consequently, if a deaf child successfully uses their implant, there may be an expectation that they will continue to exist in the hearing world, and continue to focus on spoken language. So, whilst $\mathrm{Cl}$ users may have options in terms of their involvement in $d /$ Deaf and hearing worlds, social expectation can encourage them to continue the use of $\mathrm{Cl}$ technology and to live in the hearing world.

Julie, John and Sandra's narratives on choice contrast interestingly with calls, primarily from a Deaf cultural perspective and on ethical grounds (see Hyde and Power 2006, Lane and Grodin 1997), for children not to be implanted until they are old enough to be able to make the choice themselves. These calls are also based on the ideal of the freely choosing individual self. The parents in this study, however, believed that, in the end, choosing an implant for their children was the only way to secure these choices for them later on. Julie and John expressed that, had they not chosen an implant for Hannah when she was young, she would not have had access to 'choice' as she grew up.

Julie: [...] the thing is that you can make the decision as you grow up whether you want to utilise your implant to the max, or you want to say, 'no it's not for me.' That's your decision later in life, but certainly not when you're...

John: But a decision can only be made when you have the choice. If you don't have a choice then there is no decision, because it's already made for you.

Parental decisions thus aim to 'empower' individual choice. A parent in Snapp's (2012) study also reflected on the importance of making the choice about a $\mathrm{Cl}$ early on in a child's life, noting, "it's not something you can turn around and do when he's eighteen and says 
'actually, mum, I want to hear now' you know? You don't have that chance again" (Snapp 2012: 38). Despite an emphasis on free choice, these parental narratives highlight how parents' decisions are actually made in a pressured timeframe and in the face of the limits of $\mathrm{Cl}$ technology. The desire to give them the choice of hearing further emphasises the prominence of hearing norms, however, and the explicit focus of parents on their child's attainment of speech.

Sandra also spoke about deciding on an implant as keeping the options open for Adam, particularly in terms of his being able to choose whether he wanted to be a part of the hearing world, the $d /$ Deaf world, or both. She believed that she had given Adam "an opportunity to communicate with his wider family, to communicate with his peers, to keep his options open, and that at some stage he will choose - choose to go down both, go down one, drop his implant, keep his implant." It is important to recognise, however, that choosing an implant does not simply, or neutrally, keep the options open. Whilst there is no doubt that these parents have the best interests of their children at heart, they have a strong influence on the possible experiences and likely decisions that their children will make later on. Also, all of the effort, speech therapy, money, time and energy that families invest into developing their children's $\mathrm{Cl}$ use and speech and hearing skills, as well as the habits of hearing communication that develop in family settings after cochlear implantation, implicitly emphasises the value of speech and hearing, and mean that 'dropping' the technology would be no simple step. Julie and John, in particular, spoke about the countless amounts of time and effort that was put into Hannah's speech as she was growing up.

For the parents in this study, choosing a $\mathrm{Cl}$ for their children meant having potential access to two cultures, which they saw as beneficial for their children. On this, Sandra expressed,

I just wanted to see that there's a way that Adam could stay in [our hometown], communicate in a mainstream school, but still have that side, that deaf part of him as well, like to me, I kind of liken it to being part Maori, part Pakeha, that he'd have both cultures, 'cause, you know, he'd live in a hearing and a non-hearing world, he was straddling both, that was my vision for him all along. 
Similarly, John stated that, in choosing a $\mathrm{Cl}$, they wanted to give Hannah access to both hearing and d/Deaf worlds, rather than her feeling forced to choose one. In this sense, they were attempting to make a move away from either-or ideas of involvement in $d / D e a f$ and hearing worlds. This reflects an understanding that, while speech and language acquisition may be important for these parents, a cochlear implant was not about 'fixing' their child and they were aware that their child would still be deaf. Yet for the parents, as their children grow up, both physical deafness and the $d /$ Deaf social world may represent aspects of a $\mathrm{Cl}$ user's identity that are not exclusive or all-encompassing, but rather aspects that can be part time and engaged with based on personal choice. Given that mainstream culture is largely hearing, and that parents tend to adhere to hearing cultural norms, the $d /$ Deaf world may not seem as prominent, or as requiring of a deep commitment or exclusive membership, as the hearing world does. Thus, parents may not understand how socialisation into a Deaf community takes place, nor the work and effort that it takes to be bilingual.

John also spoke of his and Julie's hopes that Hannah would be able to be bilingual, and therefore communicate in both spoken and signed languages. In this respect, he believed they were challenging misconceptions within the Deaf community that hearing parents do not want their deaf children to be involved in the community.

I think they [...] thought and probably still do that hearing people will not allow their child to liaise with the Deaf community. Nothing could be further from the truth. Right, at the end of the day the whole idea was that she could be bilingual, and to us bilingual is exactly that, two languages, one is sign language, which means communicating and living in the Deaf world, but the other one is also hearing and speaking, which is living in hearing and the other world.

It is important to recognise, however, that an individual cannot simply choose to be bilingual; instead, it takes hard work, dedication, and access to facilities to learn. Thus, whilst Julie and John wanted Hannah to become bilingual, social influences may have made this more difficult. In this respect, the lack of support for NZSL and the focus on spoken language in the mainstream would have inhibited Hannah's ability to be truly bilingual. Indeed, Hannah had access to sign language support at school through until intermediate age (Years Seven and Eight), and then did not receive it again until she was at university, where she was 65 
provided with interpreters. Before university, however, she picked up sign language again when she became involved with her local Deaf Club, where she learnt through friends. Hannah herself noted that it takes between three and six years to become fluent in NZSL, demonstrating that true bilingualism requires committed, consistent engagement with the language and people who use it.

Echoing the narratives of parents Julie, John and Sandra, both Hannah and Adam believed that their parents' decisions about Cls had given them more choices as they grew up, especially in terms of involvement in both $d /$ Deaf and hearing worlds. In narrating their life trajectories (Giddens 1991), Hannah and Adam "selected, organized, connected, and evaluated" events perceived to be important (Riessman 2008: 3), in order to present a coherent and successful sense of self. Despite some struggles and tensions arising from choices about involvement in both $\mathrm{d} /$ Deaf and hearing worlds, which will be explored later, Hannah and Adam mostly enjoyed having access to these options. Hannah stated that one obvious advantage of her $\mathrm{Cl}$ was the choice and flexibility it enabled - "you've actually got the choice, wake up one day, I wanna hear, wake up the next day, I don't wanna hear." When asked about the decision his mother had made about a $\mathrm{Cl}$, Adam was clear that he was happy with it. In particular, he thought that he had been, and would be, able to get more out of life being able to sign and speak, and therefore communicate with both deaf and hearing people. Adam sees his $\mathrm{Cl}$ as expanding his social world, and enhancing the flexibility of his identity, as he can choose which world to identify with on a day-to-day basis.

Whilst choice is often framed in modernity as having total freedom to choose, it is impossible to separate choice from the expectations of others, social influences, and the dominant paradigms - hearing norms and medical frameworks here - which one is influenced by. The significance of hearing is illustrated through the narratives of Anna and David, who spoke about their desire for Connor, their son, to be both physically and socially hearing. The desires of parents represent a further influence on the "choices" of $\mathrm{Cl}$ users, as, given that the family is generally the first point of socialisation, children are influenced by the hopes and desires of their parents. Furthermore, deaf children may want to be considered to be 'hearing' in order to fit in with their family and with social norms around hearing. As hearing parents, Anna and David wanted Connor to have access to their own 
hearing world, and therefore also to wider hearing society. Accordingly, the importance of access to the hearing world and to spoken language played a part in Anna and David's decisions about cochlear implants for Connor. On this, David expressed, "Now the fact that we're both hearing obviously had a very significant effect on how we went about things, and what we wanted for Connor." Here, while David acknowledges that their hearing status impacted on their decisions for Connor, it is also interesting to note how, in their narratives, the parents in this study divorce the influence of what they wanted for their children in making their decisions, from the notion of their children's choices. In separating these two domains of choice, and not making the link between the two, choice is presented as free and uninhibited. Both before and after Connor was implanted, Anna and David have focused on giving Connor a high level of access to sound and speech, firmly believing that this would, and will, aid him in the future. Furthermore, growing up in a hearing household, Connor was constantly exposed to hearing culture, which would have shaped his choices about the world to which he aligned himself with, as it is likely he would want to fit in with his family. Anna noted that Connor has “two older sisters who aren't at home anymore but were when he was growing up, and they were definitely beneficial, it was beneficial for him because [...] we wanted him to be hearing, [and] he saw patterns of how hearing people relate." For Anna and David, then, a $\mathrm{Cl}$ represented opportunities within the hearing world, and access to their own, and the dominant, culture.

Hannah, a $\mathrm{Cl}$ user, whilst discussing the difficulties that arise through the ability to engage with two different worlds, spoke of some of the pressures and expectations that come with implantation and its consequent "choices." As will be discussed in Chapter Seven, she referred to her liminal state between $d /$ Deaf and hearing worlds, but also spoke of the permanent nature of her implant. She stated, "even if I throw away my cochlear implant and everything, I'm still always going to be able to talk. So that kinda sucks." Hannah's assertion that not being able to rid herself of her speech "sucks" demonstrates her attachment to a Deaf identity, and that she sees speech as emblematic of a non-Deaf identity. Furthermore, her reflection on the consequence of discontinuing use of her implant illustrates that, despite having the "choice" to do so, it may not be a simple decision. The abilities that her $\mathrm{Cl}$ has helped her to develop in terms of speech are permanent, reflecting the irreversibility of decisions. Thus, I argue that it is necessary to problematise discourses of choice, and to 
recognise that choices are never entirely individualised, but always emergent within wider social and cultural spheres (Sointu 2005: 263).

\section{CONCLUSION}

This chapter demonstrates the centrality of choice as a cultural ideal in the West, and explores the narrative valorisation of choice by the participants in this study. The parents that I interviewed tended to express their desires to create strongly self-responsibilised and individualised selves in their children, selves who value and feel empowered by choice. Furthermore, the parent participants represented the choices that Cls gave their children as largely unrestrained by social and familial norms, pressures and expectations. Whilst the cochlear implant users were not always critical of the restraint inherent in these choices, they did identify some parental pressures and the impacts of them. Hannah's narrative above, for example, illustrates that she feels her identity work is constrained to an extent by the decisions her parents made about language and socialisation in childhood. Throughout the chapter, I have thus problematised choice and explored the ways in which choices are in fact deeply shaped by the prevalence of hearing norms within mainstream society, social expectations that people will adhere to such norms, or at least strive to adhere to them, and the habits and practices with which $\mathrm{Cl}$ users become familiar and comfortable. Furthermore, the choices presented in the narratives of my participants are shaped by the investments made within families in $\mathrm{Cl}$ technology and the desires that parents have for their children to function within hearing worlds. It is important to note that some parents did also express the belief that their choices would enable the development of a 'hybrid' identity vis-à-vis $d /$ Deaf and hearing social worlds - so they were aware of alternative norms and the possibility their child might have engaged with these. However, choices that lead to such alternatives are less supported and more difficult to proactively pursue. The use of life narratives here illustrates their importance in making sense and success out of life events and past decisions. Therefore, in narrating their experiences throughout interviews, my participants were not only explaining these experiences to me, but were also making sense of them for themselves. Their narratives gave coherence to distinctive events and decisions, and were "drawn from cultural and personal models for arranging experiences in meaningful ways and for effectively communicating those meanings" (Kleinman 1988: 49). Thus, contrary to Giddens' argument, for example, that modernity "offers little help as to which 
options should be selected" in the face of a diversity of choices (1991: 80), I posit that choices, in this context, are strongly influenced by social norms, pressures and expectations. 


\section{CHAPTER SIX: GROWING INTO d/DEAF IDENTITIES - ADOLESCENT IDENTITY WORK AND NARRATIVES OF SELF- FORMATION}

\section{INTRODUCTION}

How do $\mathrm{Cl}$ users navigate identities between $d /$ Deaf and hearing worlds? This chapter explores the identity work of these $\mathrm{Cl}$ users, especially in relation to their $d /$ Deafness and d/Deaf social worlds. Despite perceptions within Deaf communities that cochlear implants will erode Deaf identities and prevent $\mathrm{Cl}$ users from becoming involved with the Deaf world, the $\mathrm{Cl}$ users in this study emphasised the importance of becoming part of various $d /$ Deaf social spheres. This chapter demonstrates that, despite cochlear implants being a technology designed to 'normalise' deaf children, to minimise hearing 'disability,' and consequently enable increased involvement in the 'regular,' hearing world, $\mathrm{Cls}$ do not in fact elicit such an easy involvement in mainstream, hearing worlds. The participants in this study demonstrated that their Cls did not make them 'hearing,' and expressed a strong need to explore $d$ /Deaf social worlds in their teenage years, which is common amongst deaf people raised in hearing families (see Smiler and McKee 2006, Valentine and Skelton 2007, Sheridan 2008, Leigh 2009). For the participants in this study, having a $\mathrm{Cl}$ necessitated ongoing identity work in relation to their deafness, and the role of both $d /$ Deaf and hearing worlds in their lives. To explore the identity work of my participants, I draw on Snow and Anderson, who conceptualise identity work as "the range of activities individuals engage in to create, present, and sustain personal identities that are congruent with and supportive of the selfconcept" (1987: 1348). Such identity work may involve the alteration of personal appearance, selective association with particular individuals or groups, and the verbal construction of personal stories of the self (Snow and Anderson 1987). Thus, in focusing on the identity work of these $\mathrm{Cl}$ users, I also draw on concepts of self-narratives. In this respect, my interest in "narratives and the narration of identity signifies [...] a stress on the [...] uncertain and creative processes of construction and fabrication" (Byrne 2003: 30). The narratives of my participants therefore demonstrate the fluidity of their identities, and the constant work in forming and creating identities that fit with their involvement in various social worlds. 
I understand identity as a fluid project which is worked on throughout one's life span, and which incorporates both internal and external perceptions of the self. I draw here on Martha Sheridan's comprehensive understanding of identity:

Identity is a process that develops and changes over time, involving many aspects of one's biopsychosocial and spiritual systems. It is constructed from our understandings of the biological [...], psychological $[\ldots]$, social $[\ldots]$, and spiritual $[\ldots]$ aspects of our beings. It is also a compilation of our interpretations of our experiences in the past, our selves and experiences in the present, and our images of what is possible for us in the future [...]. It is a symbolic-interactive process of mind, self, and society [...]. It involves our self-perceptions, which lead to self-definitions (covert identity), as well as the perceptions that others have of us and the identities they assign to us (overt identity) (2008: 188-9)

I therefore show how identity work is a reflexive project which is multidimensional and draws on both cultural knowledge and the ability to perform culturally appropriate roles (Leigh 2009).

This chapter addresses the changes that the $\mathrm{Cl}$ users in this study have experienced in terms of their identities, for which I use Giddens' (1991) concept of the reflexive project of the self as a frame of reference. Given the importance of participants' involvement in both $d /$ Deaf and hearing worlds, I draw on their narratives about their experiences of belonging to these two worlds, and the inherent complications and tensions that come with such belonging. I also draw on my participants' narratives on the importance of interactions with self-same peers - others who are d/Deaf, but who do not necessarily have cochlear implants. Thus, it is $d /$ Deafness which draws them to others and which elicits a connection, not their use of this technology. In this respect, I explore the space that these $\mathrm{Cl}$ users occupy in between $d /$ Deaf and hearing worlds, arguing that they occupy a liminal space, as they are not fully immersed in either world. 


\section{Establishing a Place in the d/DeAf WORLD}

The concept of a Deaf culture was articulated in the 1970s, with d/Deaf individuals celebrating the uniqueness of their cultures and languages, and beginning "to see themselves as belonging to a linguistic minority rather than a group of people bonded through disability" (Bauman and Murray 2010: 211). This concept represented

an emerging voice that encompasses cultural explicitness, selfconsciousness, and a centredness around a signed language that was not previously reflected in the self-perceptions of deaf individuals. The values, mores, and ways of relating that have emerged out of this background are transmitted from Deaf parents to their deaf children and from Deaf peers to those deaf individuals who become exposed to Deaf culture at varying stages in the process of life (Leigh et al 1998:

The $\mathrm{Cl}$ users in this study, born to hearing parents, were exposed to Deaf culture through Deaf peers, and predominantly in their teenage years. Given the often-cited sense of belonging $d /$ Deaf individuals feel when they encounter Deaf culture later in life, $d /$ Deaf individuals may be inclined to incorporate Deaf culture into their identities, and consequently into their narratives of the self. In doing so, many begin to experience a "confidence and sense of self worth about being D/deaf and being part of the D/deaf community" (Skelton and Valentine 2003: 464). The process of incorporating deafness into one's identity may involve an exploration of the many labels assigned to deaf people - for instance, 'Deaf,' 'deaf,' or 'hard of hearing.' Whilst an individual's choice as to which label they eventually adopt is influenced by external factors, the $\mathrm{Cl}$ users in my study spoke of their choices to identify as 'deaf,' or 'Deaf,' or combinations of the two. In this sense, depending on the context of any social interaction, an individual's perception of their own identity may change, with particular aspects drawn out and emphasised. Therefore, it is important to note that their narratives of the self, and thus their self-identities, are subject to change, particularly depending on place and time (Leigh 2009: 62).

Hannah and Adam, who were implanted at the ages of five and three, respectively, were clear on their current self-identifications as Deaf. Hannah, who, as illustrated earlier, moved 
through various stages of identity in terms of being hearing impaired or deaf, now definitively identifies as Deaf, stating, "I'm definitely capital ' $D$,' there's no doubt about it." Similarly, Adam perceives himself to be Deaf: "Frankly I consider myself Deaf because I sign, I understand the deaf issues and so on." Jack, on the other hand, was not so clear about how he currently identifies, but rather emphasised his involvement in both $d /$ Deaf and hearing worlds, as will be explored below. Jack was clear about his acceptance and recognition of his deafness, but did not appear to claim a specifically Deaf or hearing identity status. Instead, his deafness forms a part of his identity, and has facilitated his involvement in a second community. For Isaac, who was implanted at age 12 , his current identification as either hearing or deaf is more complicated. He expressed,

[E]ven now, if you asked me if I considered myself hearing or deaf, I would have to think about it. Because physically deaf, but mentally hearing, if that makes sense. I see myself as a hearing person, if I dream about myself, then I'm a hearing person in my dreams, that kind of stuff. So I have an active perception of me as being a hearing kid, even though I'm deaf and I'm fully aware of that as well [laughs]. So it's a bit of self-denial I guess [laughs] [...].

Additionally, he noted, "I probably fit under the little ' $d$ ' category, but again I consider myself hearing, so yeah. It's a real philosophical mindbender - who am I? [Laughs]" Isaac's reflections on his identity highlight the complicated space that $\mathrm{Cl}$ users can occupy. He is aware of his deafness and comfortable with it, as well as being actively involved in the Deaf community and conversant in sign language, but he also considers himself to be hearing. As will be explored, Isaac's ambivalence about his d/Deaf or hearing status reflects his desire to be involved in the wider hearing world. Additionally, his narrative here demonstrates a particularly remarkable degree of self-reflexivity, and thus a profound engagement with his reflexive project of the self (Giddens 1991).

\section{Identity Work and the Formation of an Authentic Sense of Self}

Two of the $\mathrm{Cl}$ users, Hannah and Jack, spoke about earlier self-perceptions of their deafness, including presentations of the self and self-assigned labels. Both Hannah and Jack recalled times in which they downplayed their level of hearing loss, and represented themselves as 73 
hearing impaired, rather than deaf. For Jack, his use of hearing aids assisted in this perception of himself, as he believed that the technology raised his hearing to a "good" level. Hannah remembered being embarrassed by her deafness, to the extent that she would try to hide it in some situations. Whilst representative of her own perceptions of her deafness and its place in her identity, this also reflects interactions with others and the influence that these have on subsequent self-labelling. Here, Hannah spoke of her engagement in identity work, as she endeavoured to present a particular image to others; one which emphasised that she was 'hearing-impaired' rather than 'deaf.'

Hannah: I was hearing impaired. You know I had problems with my hearing, just, 'you just need to speak up', but there's no issues about it [speaking ironically]. So I was like that in primary school, and all the boyfriends, everything I had were hearing. I was embarrassed of being deaf [...]. Before fifteen, I was like, 'oh no, no, I missed what you said,' I would just pretend, laugh along or you know... But now I'm like, 'I missed that - I'm deaf. What did you say?' I'm just real straight up, because before I was quite timid, because [...] I didn't want to look stupid [...].

Thus, in the life stage that Hannah recalls here, she attempted to highlight the 'hearing' aspects of her identity. This involved portraying a particular identity to others, and was likely reflective to some extent of the stigmatisation surrounding deafness. In this sense, Hannah demonstrates that her identity formation at this stage in her life was centred on fitting into a mainstream hearing world, and being accepted by hearing peers.

Hannah expressed, however, that her perceptions of her deafness changed a number of times, referring to these changes as "identity crises." This demonstrates that identity, particularly throughout adolescence, is fluid and a constant project. Maxwell-McCaw et al state that, "[a]s new information about oneself emerges, there is often a process of identity restructuring," and that this process continues throughout the life span (2000: 2). Hannah's involvement in both $d /$ Deaf and hearing social worlds complicated how she thought about her deafness and how she identified herself in regards to this. She noted though, that, especially being in mainstream education, she lost touch with the $d /$ Deaf part of her identity and became immersed in hearing social worlds. 
Hannah: I had so many times where I had [a] crisis with my identity, whether I was hearing impaired, or deaf. I switched back and forth, especially being mainstreamed - you know, you lose [...] all your deaf friends [from her time at a deaf school]. I used to spend my weekend with my deaf friends, but when I made more friends in [mainstream] school, I spend my weekends with them. So by the time I was probably thirteen or fourteen, [...] I didn't know how to sign, I was just not deaf. Well I am, but at that time, I wasn't. I didn't identify myself as Deaf with a capital 'D.' And it wasn't till I was fifteen - [...] I was bored one Friday night, so I thought, 'I'll just go and check out Deaf Club.' It was down the road, there was no excuses. My parents were encouraging me to go. So I went there [...].

Hannah's renewed involvement with the Deaf community in her mid-teens signalled a change in her perceptions of her deafness. Through increased interactions with other $d /$ Deaf people, Hannah became more accepting of and assertive about her deafness. She noted, "After going to Deaf Club at fifteen - probably around seventeen, eighteen, that's when I started being quite assertive, being like, no 'I'm proud of myself. Yeah I can't hear as well as you guys, but that's ok - I can still do other things just as good as you, if not better."' Thus, interactions with $d /$ Deaf peers helped to solidify Hannah's d/Deaf identity, increasing her confidence throughout the process.

In her project of the self, Hannah is continuously revising her biographical narratives, especially given the various social worlds she is engaged with. As Giddens states, selfidentity "forms a trajectory [...]. Each of us not only 'has', but lives a biography reflexively organised in terms of flows of social and psychological information about possible ways of life" (1991: 14). Yet as Hannah's narrative indicates, identity is ideally somewhat solidified throughout adolescence in our cultural context, as individuals experiment, and begin to 'discover' who they are and where they 'fit' in the world. Throughout adolescence, these $\mathrm{Cl}$ users began to make decisions about which social worlds to interact in and which behaviours to adopt accordingly. 
For Jack, acceptance of his deafness came after he had received his cochlear implant, as he recognised that the technology did not rid him of his deafness. Therefore, rather than resulting in the loss of any sense of attachment to deafness and Deaf culture, receiving a $\mathrm{Cl}$ prompted Jack to make connections with the d/Deaf world and to incorporate $d /$ Deafness into his self-narrative. Jack noted, "It wasn't until I got my implant that I started recognising that I was deaf, because as soon as the magnet comes off, or there's a malfunction, I don't have any hearing, so it's [...] like just vanish and come as it likes." He also said,

Yeah I guess I, probably in those first couple of months, just after the operation, when I was thinking about it, I was like, 'I'm pretty sure hearing should be a bit easier than what I'm going through right now,' and then you get it, and then when you take it out, you're like, 'cause I've still got a little bit in my left ear, I'm like, 'hmm really wasn't hearing much at all, was I?' [Laughs] So yeah [...] I guess it wasn't much of a change to say that I was deaf - it wasn't like an instant thing, it was like a beginning to self-realise. But now I accept the fact and move forward with the fact that I [...] have two worlds almost.

Thus, contrary to misconceptions about the abilities of cochlear implants to make deaf people 'hearing,' it was this technology that allowed Jack to come to terms with his physical deafness. Receiving a $\mathrm{Cl}$ also meant that Jack became aware of the limits of the technology, and thus the long term reality of his hearing impairment. After implantation, Jack worked through how to incorporate his deafness into his identity. In some senses, accepting his deafness allowed Jack to see himself in a new way and conceptualise how he could be involved in two worlds. 'Accepting' one's deafness, then, as Jack's narrative suggests, implies reflexively constituting an increasingly settled and 'coherent sense of self' (Giddens 1991:100) in these years.

Adam, a $\mathrm{Cl}$ user who grew up in a small New Zealand city, spoke of the emergence of his $d /$ Deaf identity at high school, and his consequent desire for interactions with other $d /$ Deaf people. He was the only deaf person at his schools throughout his education, however, and such a lack of access to similar peers led to social and personal worries for Adam. 
At high school, [...] my deaf identity [was] starting to come out, and I was just like, I'm deaf, everyone else is hearing. I would like to talk to a deaf person about being deaf, because [they would] understand more and there was just no one around to talk to, so I kinda got a bit depressed over that. I had some deaf people on Facebook I could talk to, but it's not the same on Facebook, 'cause you're not really signing or just doing Deaf culture-y kinda stuff, so if there was like a deaf youth group in [my hometown], l'd be really happy.

Adam's desire for involvement with other deaf people is reflective of one of the realities that deaf and hard of hearing people present - the desire for self-same relationships (I draw on the term 'self-same relationships' from Sheridan (2008); see also Leigh 2009). This term, meaning "deaf like me" (Sheridan 2008), is useful here in reference to the sense of ease in communication and social relations that deaf people often encounter when they meet others like them. As peer relationships become increasingly important in adolescence (Sheridan 2008), deaf individuals may increasingly seek out others who are like them and who share an understanding of experiences of deafness. In particular, "[f]or mainstreamed or isolated deaf persons, there is often a sense of coming home" when contact is established with other deaf people (Leigh 2009: 48). Adam's wish to talk to other deaf people who would understand his own experiences reflects his realisation of being different to the people with whom he interacted daily in the wider hearing world, and a consequent need to be surrounded by people like himself.

\section{Belonging in the d/Deaf World: Identification with Self-Same Peers}

The $\mathrm{Cl}$ users in this study spoke positively of interactions with d/Deaf peers, and of making connections with the Deaf community in their teenage years. Thus, self-same relationships play an important part in the socialisation of $d /$ Deaf individuals, and constitute an important part of their Deaf identities. For these $\mathrm{Cl}$ users, becoming involved in Deaf communities felt easy and comfortable, especially in regard to communication and identification with others who have had similar experiences of being deaf in the hearing world. Isaac spoke of his affinity with the d/Deaf world, expressing, 
I am pretty drawn to it [the $d /$ Deaf world], because it's more natural. Like...I don't know, it's like Tarzan being raised by the apes - I mean sure he can be as much of an ape as he likes, but you put him with humans, he's going to be drawn to acting and behaving like a human. Exactly the same in my situation, where it's a lot easier for me to go to deaf things and be a member of Deaf society than it is for me to be a member of hearing society. Yeah, because everything in the Deaf society everything is geared toward people like me, so lighting is fine, the communication is the way I want it, I don't have to listen actively to what they are saying because they're signing so, and I am quite a visual person and I do rely on body language a lot [...].

Isaac's narrative naturalises Deaf identification, and reinforces the notion that a $\mathrm{Cl}$ does not make a deaf individual either physically or psychologically hearing. Instead, these individuals tend to gravitate towards the $d /$ Deaf world, or at least the company of deaf peers, at some point in their lives. Likewise, Anna and David spoke about their son Connor's affinity with other $\mathrm{Cl}$ users in his experiences at an international camp for young individuals with implants. Anna noted, "it was kind of a sense of, there are people like this round the whole world, $[\ldots]$ who are struggling with these same issues. And [...] I think he stopped feeling alone, didn't he?" These examples demonstrate that interactions with similar peers are often found to be a welcome retreat for $\mathrm{d} /$ Deaf individuals, as these people have also experienced what it is like to be a member of a minority group in wider society. Such examples reflect how self-stories are always embedded within social identities and networks.

Adam also spoke positively of his involvement with the d/Deaf world, especially in meeting people "kinda the same as me," who knew what it was like to be deaf or to have a $\mathrm{Cl}$. $\mathrm{He}$ attended short camps at van Asch Deaf Education Centre while he was at school, which "attempted to bring mainstream deaf people to the deaf world, [...] and we just learned how to sign a bit better, learned a bit of Deaf culture, meet new deaf people." Such camps would have served as opportunities to experience belonging with self-same peers, in turn influencing the identities of the young deaf individuals attending them. On his experiences at these camps, Adam expressed, 
I remember 2010 - the two highlights of that year was going to Australia for holiday and going to van Asch, and 2011 was just going to van Asch, because it was awesome. Like I'm outgoing but I never actually understanded [sic] people, and because everyone was the same as me - they had cochlear implant, they couldn't really sign that well, but they knew what they wanted from other people communication wise, [...] we had no problems understanding each other, we talked a lot. It was just different from being at high school, where I'm just like, 'I don't know what's going on, I'm out.' So [...] it's a good way of feeling involved, socially involved.

As was the case for Isaac, Adam felt an easy affinity with the d/Deaf world and with deaf peers and, at the camps at van Asch, felt involved, included, and 'normal,' in contrast to his experiences in mainstream education. Identity work in adolescence therefore involves individuals valuing and making decisions about social belonging, connection and association.

Having recently returned from competing at an international deaf sporting event when he was interviewed, Jack also spoke positively of experiences being surrounded by other deaf people. He spoke of his experiences at the sporting event as being affecting, as the event gave him access to people similar to himself; he noted, "it was just cool to see all these hundreds of athletes who sign and enjoy sports. [I]t was sort of emotional I guess." Furthermore, he expressed,

I just got back from the [sporting event], which just opened up your mind as to how many other individuals that are around the world who go through the same thing you do. And like sport we've got in common, and so it was just cool to be around similar minds. 'Cause even though there's deaf people in the country [New Zealand], they might not be as fanatical about health or whatever, so it's hard to find that medium, that same interest topic almost. But yeah, [...] we had quite a small team, so I'm hoping to get involved with Deaf sports more to increase this team, or just to bring like-minded people together in New Zealand, like the [sporting event] does for the world, sort of thing. 
Jack also spoke about affinity with the d/Deaf world in terms of communication, as, for him, communication in this world requires less effort and concentration. On learning NZSL at university, he stated, "It was great. [I]t was almost relieving in a way, [...] there's no talking, and there's no talking in the classes, and it was just quite [...] a load off my shoulders almost, to just go into an environment where there's no talking." Thus, Deaf visual modes of communication, along with the peace of the $d /$ Deaf world in comparison to the noise of the hearing world, were a welcome relief for Jack, who, despite being clear about the positive effects of his $\mathrm{Cl}$, still finds communication and relationships in the auditory world to be challenging at times.

\section{BELONGING IN THE HEARING WORLD}

Given that the $\mathrm{Cl}$ users in this study were born into hearing families, and into societies in which hearing is the norm, access to this social world was assumed to be a given. However, in the hearing world, given its frequently inaccessible nature, deaf individuals face communication, language and cultural barriers (Sheridan 2008). For these $\mathrm{Cl}$ users, such difficulties tended to present themselves primarily in the forms of communication and socialisation, as has already been demonstrated in Chapter Two. Sandra spoke of the difficulties that Adam has faced socially with hearing peers, noting how tough it has been for Adam, particularly as he reached his teenage years.

Sandra: [...] as he got older and older, and people started moving into, I don't know, looking at girlfriends, that whole other social side of going out to dances or clubs or movies or that thing, he just got less and less involved because it's very challenging for him, and so he became quite isolated I felt. I really felt for him, about Year 12, he just really did go downhill quite a lot - I think he was just totally lost, and he got through Year 11 with school, and then socially people just started going their own way and doing their own thing, and just changing, as you do at that age, you know, [...] and Adam just never sort of fitted.

Sandra's worries for Adam highlight that, whilst $\mathrm{Cl}$ users may be able to interact with hearing people and participate in wider hearing society, this does not automatically equate with social inclusion or feelings of belonging. Consequently, $\mathrm{Cl}$ users can encounter experiences 
of isolation, especially once they are aware of their deafness and the part that this plays in their identity. Such feelings might contribute to difficulties in incorporating aspects of their experiences in the hearing world into concepts of the self and biographical narratives. Furthermore, this can contribute to the positioning of $\mathrm{Cl}$ users in a liminal space between $d /$ Deaf and hearing worlds, as they attempt to negotiate peripheral social spaces.

Family plays a key role in socialisation into the hearing world, and in desires to be seen as a 'hearing' person. Although he is aware of the contradictions and is drawn to be a part of the $d /$ Deaf world, Isaac considers himself to be hearing and favours involvement in hearing worlds. He credited his parents on their decision to treat him as a "hearing kid," especially because it meant he could be included in his family.

[O]bviously my parents, when they found out that I was deaf, [...] they obviously made an active decision to treat me as a hearing kid, with hearing aids. And yeah, I think that's the way I like it. I mean they could've, alternatively, all learned sign language, treat me as a deaf kid, but that would just of made me more excluded, to be honest, 'cause they could speak and I can't, which would of created the whole division. So to raise me as a hearing kid was a really good decision, so props to the family [laughs].

Furthermore, in terms of his own desires to be hearing, Isaac spoke of the influence of his family.

No doubt about it, they [family] would have been like a major influence, 'cause you're growing up with these people, they're your family, so you kind of try to fit in with them as much as possible, and if everyone else in the family is hearing and they're all treating me as hearing, then, you know, I'm hearing [laughs]. So [...] some of it would have come from me being like, 'I want to be treated as a hearing person' - that's how they're treating me, so it's fine.

Isaac's narratives illustrate how $\mathrm{Cl}$ users need to navigate not only peer inclusion, but also family inclusion. Close family relationships can influence the place that the hearing world occupies in the identities of $\mathrm{Cl}$ users. Through his family in particular, Isaac has established a 
sense of belonging in the hearing world, and has thus incorporated this world into his narratives of the self. However, his involvement in $d$ /Deaf and hearing worlds reflects a desire to belong to multiple groups, and perhaps his family's implicit acceptance and support of this "choice." Therefore, Isaac highlights that both his deafness and his affiliation with the hearing world contribute to his project of the self.

Anna and David spoke of their own wishes that their son, Connor, be a part of the hearing world. For them, this means bringing Connor up in their own culture, and in a world in which they feel comfortable. Anna and David defined Connor's current involvement in the hearing world through who he socialises with, David noting, "All of his mates are hearing, normal. So he's in the hearing world." Here, David associates being hearing with being 'normal,' an indication perhaps of his desire for Connor to be perceived as hearing and a member of mainstream society. Thus, the narratives of Anna and David emphasise the importance of the hearing aspects of Connor's identity, and, in the process, downplay his deafness. It is important to note, however, that Connor was only 15 years old at the time of the interview with his parents, and that he is therefore still negotiating the adolescent phase of his identity work, in which he may, in particular, experiment with different $d /$ Deaf and hearing social spheres in which he feels he belongs.

\section{CONCLUSION}

The cochlear implant users in this study demonstrated, through their narratives, that they have forged identities through multiple threads and factors over time, but particularly throughout adolescence. Social influences and norms from both d/Deaf and hearing worlds, parental hopes and expectations, desires for connections and a sense of belonging, as well as individual identity trajectories have contributed, and continue to do so, to the identity journeys of these young individuals. Through selective association with particular individuals or groups, self-representation, and the verbal construction of identities through narratives such as those told in my interviews with these participants, the $\mathrm{Cl}$ users in this study are constantly engaging in identity work to form coherent, authentic self-identities. However, forging identity through multiple threads has complicated their biographical trajectories in relation to their belonging between and within $\mathrm{d} /$ Deaf and hearing worlds. 


\section{CHAPTER SEVEN: THE BEST OF BOTH WORLDS? \\ NAVIGATING LIVES BETWEEN DEAF AND HEARING \\ WORLDS}

\section{INTRODUCTION}

While in the last chapter I explored the identity work undertaken by the $\mathrm{Cl}$ users in adolescence, which often involved 'realising' and acknowledging deaf identity, in this chapter I will show that these individuals are constantly navigating lives between $d /$ Deaf and hearing worlds. I argue that these $\mathrm{Cl}$ users occupy a liminal space between these two worlds, and that they are in the process of working out a way in which to incorporate aspects of both worlds into a coherent sense of self. Thus, whilst I have no doubt that they have indeed formed hybrid identities to an extent, and that their identities are fluid projects, I argue that they are in a long-term state of liminality as they are not fully incorporated into either d/Deaf or hearing worlds. However, while involvement in these two worlds has elicited tensions and some struggles to belong in both spheres, the $\mathrm{Cl}$ users in this study were also positive about having the 'best of both worlds' and aware of the benefits of this double involvement.

InVolvement in TWo Worlds: Adding Richness to IDENTITY

Hannah and Adam spoke of having 'the best of both worlds,' reflecting their positive outlook on their involvement in both the $d /$ Deaf world and wider hearing society. Adam expressed, "Yeah I feel like I [...] got more out of my life being able to hear and being able to sign at the same time. [...] I still have a Deaf identity - I speak to d/Deaf people, I speak to hearing people. It's like I have [...] the best of both worlds." Here, Adam recognises that $d /$ Deaf and hearing worlds can offer him different opportunities, especially in the way of social interactions, which may be particularly salient given the small size of $d / D e a f$ communities in New Zealand. In this sense, having a $\mathrm{Cl}$ does not stop Adam from interacting with other $d /$ Deaf people, but it has also improved his abilities to interact within wider hearing society. 
He stated, "I like to be able to communicate with hearing people and deaf people as well. It's like you've got a stronger identity because you've got two in one." Thus, for Adam, having access to two worlds has added richness to his identity, especially through his ability to interact with a wider range of people from different backgrounds. Similarly, Hannah spoke of feeling "blessed" in that her $\mathrm{Cl}$ enables her to be a part of, and interact in, both $d /$ Deaf and hearing worlds if she wishes, and allows her to move between the two. Despite initial difficulties with being part of both $d /$ Deaf and hearing worlds, especially in terms of confusion in dealing with how this affected her identity, Hannah conveyed that she is now comfortable with being involved in two communities.

Hayley: So do you feel [...] quite comfortable in both worlds?

Hannah: Now, yes. Back then, you know, I hated it - get confused, you know, I just want one. But now, I'm happy. Because, you know, I'm older now, I'm in my early 20s, I can handle it a bit better. Like being a teenager - you know, you're inexperienced, you're dealing with the hormones, you're changing, you don't want to change, deal with two identities. But now, I'm calm, you know, I'm normal, and I only have to deal with that. [...] I think I like it that way. I mean, just because now I experienced having two different ways, just being one way, to me would be boring.

Hannah's assertion here is reflective of the increasing prevalence and acceptance of hybrid, multi-layered identities in modern society. According to Keri E. Iyall Smith (2008), hybridity was initially a term of disparagement, but its meaning is changing in the globalised world. She states "identities for all individuals and collective selves are becoming more complex. With globalization and increasing modernization, being a hybrid is now a benefit" (Smith 2008: 4). Having worked through the difficulties of dealing with her place in two worlds, Hannah sees this involvement, whilst complex, as adding to her identity.

Jack was also positive about his involvement in these two worlds, and spoke of this especially in terms of having access to two languages, which he deems to be beneficial. He views his ability to use two languages as a proficiency that most people do not have, stating, "it's like a skill almost, [...] I can talk English, and I can sign New Zealand Sign Language, and 
most people can either talk English or sign New Zealand Sign Language, so it's [...] like another, what do you call it, weapon in the arsenal [...] to have disability, to understand two languages [...]." Furthermore, this skill contributes to his ability to be part of both $d /$ Deaf and hearing worlds, and negotiate the barriers between the two. He expressed, "now I can sign, so it means that [...] I sort of almost have two worlds, where I can have one foot in both. I can hear if I want to, which is very good, not perfect, but I can also sign, where I don't need to hear, because that is part of who I am." Jack highlights that, despite his $\mathrm{Cl}$, his deafness (as symbolised by the use of NZSL) is still a fundamental part of his identity, but that his $\mathrm{Cl}$ has also enabled him to maintain a life trajectory that incorporates involvement in the hearing world and wider society. He was positive about what both the d/Deaf world and the hearing world could provide for him, especially in terms of opportunities and relationships. On this, Jack said, "I understand that having a cochlear will grant me a better chance in jobs and relationships, but at the same time I'm not ashamed of being deaf, it's like I got this so then I could continue on the way I was growing up, but I also have to accept that I'm deaf, but it's cool, it's like there's a community of [d/Deaf] people out there who know what you go through [...]." Thus, for Jack, involvement in both of these worlds is important for his sense of self and identity, with both worlds providing unique opportunities and different frames of belonging. In particular, involvement in the d/Deaf world provides him with the opportunity to meet and interact with self-same peers who can provide a sense of comradeship and identity through the shared experiences of being deaf.

Whilst the remainder of this chapter focuses on some of the more complicated aspects of occupying a liminal space between $d /$ Deaf and hearing worlds, it is important to recognise the possibility that individuals who occupy this social space can also be in a relative position of power. In this respect, $\mathrm{Cl}$ users who can sign and are confident in using spoken language are able to take part in the social spaces on either side of the 'boundary,' and may gain power or status in being able to mediate between the two worlds. Therefore, it is possible for these individuals to use the liminal experience and their liminal social positioning to their advantage. In some respects, then, this positioning can be reflective of a hybrid identity, in that individuals may incorporate their "ability to negotiate across barriers - language, cultural, spiritual, racial, and physical" as an asset in their identity formation (Smith 2008: 4). Moreover, in that my participants highlighted having 'the best of both worlds,' it is important to recognise that their occupation of this liminal state does not always denote 85 
being alienated from self and society, but rather a sense of empowerment. Thus, whilst these $\mathrm{Cl}$ users did identify some of the difficulties of being 'in-between,' they coherently incorporated such difficulties into their self-narratives (Giddens 1991).

\section{Moving TOWARDS BELONGING IN THE D/DEAF WORLD}

Whilst all four $\mathrm{Cl}$ users in this study currently have social connections in both $d /$ Deaf and hearing worlds, and speak positively of this dual involvement, this was not always the case. Although parents desired this involvement for their children, it does not seem that this occurred as these $\mathrm{Cl}$ users grew up. This may be, in part, reflective of the difficulties that parents also face in adjusting to a child's deafness. Sheridan states that "[h]earing parents who have no previous experience with deaf people go through their own developmental issues as they grow in their understanding of their deaf child and Deafhood. A dual development process takes place - that of the family in response to its deaf member(s) and the child's development in the context of the family and society" (2008: 22). Furthermore, providing access to the $d /$ Deaf world is not always an easy task, given the relatively small size of Deaf communities in New Zealand, especially in small centres. Sandra, Adam's mother, spoke about the difficulties she faced in getting Adam access to other deaf children of a similar age when he was growing up: "It's something that I always felt frustrated I couldn't give him. I can give him an education side and help him through anything he needed, but that Deaf culture side had to come from being immersed in it."

All four $\mathrm{Cl}$ users in this study had encountered some challenges since becoming involved in Deaf communities. The experiences that these participants spoke about in interviews were primarily in relation to uneasiness surrounding their Cls within Deaf social spaces. However, it is important to note that none of these $\mathrm{Cl}$ users were excluded because of their implants, but sometimes treated with some level of hostility, given the sensitive nature of cochlear implantation for some within Deaf communities. When asked whether she had encountered difficulties in the Deaf community with her $\mathrm{Cl}$, Hannah replied:

Oh yeah! You know, sometimes I could be talking in a conversation, like signing, and someone drops a glass - l'll look away, go 'ooh what was 
that sound?' They'll be like, 'you can hear, you're not deaf'. I'll be like, 'yes I am, I am deaf' [firmly]. They go, 'you just heard that, you're not deaf.' I'm like, 'I have a cochlear implant.' They'll be, 'Turn it off! We're in deaf club.' You know, sometimes they do criticise me about it, but I mean, [...] that's quite rare. I think only like twice I've ever encountered that. The rest they really just don't care - if I can talk to them in sign language, that's good enough. You know, [...] I'm not forcing them to talk, which I would never do. I think that's what they're all worried [about], 'oh she can hear, she's going to make sign language die - she's going to force us to talk.' No.

Hannah's narrative here illustrates some of the concerns prevalent within the d/Deaf world about the effect that $\mathrm{Cls}$ could have on their communities. Such concerns stem from the long history of oppression that $d /$ Deaf people and communities have been subjected to, particularly in the forms of eugenics and oralism. According to Lane et al, the oralist philosophy, which denigrates sign language and encourages spoken language, has resurfaced with the advent of $\mathrm{Cls}$, and is now "backed by all the authority of the modern medical establishment. Once again, the parents of Deaf children are advised not to let their Deaf children sign, if they are to be or have been implanted" (1996: 372; see also, Padden and Humphries 2005). Despite such concerns, however, Hannah noted that she has not encountered much opposition to her $\mathrm{Cl}$ within deaf communities. This could be a reflection of the softening of attitudes to Cls within the deaf world, as addressed in Chapter One.

\title{
'BetWixt AND BETWEen'
}

\begin{abstract}
Whilst participants with Cls believed that their access to two social worlds has provided them with more choices and opportunities, it was also clear that the practical task of incorporating two sets of social networks into their lives has not always been straightforward. Indeed, involvement in both of these worlds can entail struggle and tension. At times, this tension can be complex and demanding, especially in choosing, establishing and maintaining connections in these two worlds. Hannah, a $\mathrm{Cl}$ user, spoke of such issues:
\end{abstract}


I mean, some days I love having the best of both worlds. Other days, I'm like, I actually cannot involve myself fully in one world, I only can have a part. You know, I cannot...it's kind of an overlapping - I'm in that bit there, I can't step out and be hearing, and I can't step into...well I mean down the line if I decided to screw everything and chuck away my implant, I probably could, but you know that's thousands of dollars and hours and everything and...I like being able to hear, having the choice to hear. So it's kind of like I actually, I can't step into hearing, like that's impossible, I can step into the deaf, but I still can talk quite well, so even if I throw away my cochlear implant and everything, I'm still always going to be able to talk. So that kinda sucks. But at the same time, I'm like I have both worlds [laughs]. So it depends what mood I'm in on that day! [Laughs].

Hannah's reflections illustrate both the positive aspects of having access to these two worlds, and the difficulties or frustrations that can arise. In this respect, she values being able to be involved in both $d /$ Deaf and hearing social worlds and understands that each world offers unique experiences and different social opportunities. At the same time, however, she is aware that, given her increasing involvement in Deaf culture, her $\mathrm{Cl}$ has given her something that she can never rid herself of - speech. Her speech abilities may be viewed negatively within Deaf culture and may hinder her involvement or inclusion in the Deaf world. This demonstrates Hannah's liminality within Deaf culture, as her implant serves to differentiate her from other $\mathrm{d} /$ Deaf people, and therefore situates her on the periphery of the $d$ /Deaf world. Thus, while the $\mathrm{Cl}$ users in this study are embedded within hearing culture and ideas of the 'normal,' hearing body, they are also confronted with ideas of the $d /$ Deaf body - one in which hearing and speech is not necessarily celebrated.

Adam also spoke of how Cls can have a negative impact on d/Deaf individuals' involvement in the d/Deaf world. Now at university, Adam has gained more access to other deaf people and the Deaf community, but had little access to this community when growing up. Accordingly, whilst he can now appreciate being a part of both worlds, this was not always the case. On this, he spoke about some of the positives and negatives of cochlear implants. 
[T] he positive is that now deaf people can get more involved in the hearing world, but at the same time it puts them off $d /$ Deaf world, because like if you get it at a young age, parents will encourage them how to speak, but they'll forget to encourage them to do sign, so they can't sign to deaf people. They'll go to a mainstream school, they'll be raised hearing and won't have much knowledge of the Deaf culture, like, for example, me - I started getting involved this year, that's 18 years I've been hearing. But if I was born deaf and I didn't have a cochlear implant, I would be 100 per cent immersed into [the] Deaf world, but no knowledge of the hearing world. [...] I wouldn't know the social protocol for talking to a hearing person. So it's got a lot of positive and negative changes to the Deaf community.

Adam's statement that he has been hearing for 18 years reveals an important aspect of his identity: $d$ /Deafness did not play a part in his self-identity until recently. Furthermore, it also perhaps reveals that parents may not attribute such centrality to deafness within identity as their deaf children eventually do, and that aspects of identity drawn from hearing social worlds may take primacy within the family. In the last part of this quote, Adam reflects on how his $\mathrm{Cl}$ has enabled him to learn how to conduct himself in the hearing world. Although he hopes that $\mathrm{Cl}$ users will be exposed to Deaf culture and sign language from an early age, he is still positive about the opportunities for interaction with hearing people and within hearing society that the technology provides.

Yet switching between worlds is not easy work. $\mathrm{Cl}$ users' narratives demonstrated the difficulty of merging a multiplicity of threads and influences into one identity, and the difficulties of existing as a mediator between $d$ /Deaf and hearing worlds. On this, Adam explained:

I don't really find it hard being part of both, because I have no problem being part of the hearing world, apart from missing out on conversation and stuff. It's more like, I find it hard involving myself in the d/Deaf world, 'cause I'll try interact in a hearing way, and deaf people don't like that because [...] they're strongly independent. If I say something like, 'I can talk to hearing people - let me help you translate,' they'll be like, 
'fuck off I don't want your help.' But if I say something like, 'do you want me to help you? Do you want me to help support you to communicate with the hearing world?' And they might be like, 'oh that's supporting me, you're not helping me,' and then I'll be able to translate for them because they feel like they're in control, they're not being weakened, so yeah.

The uneasy interactions recounted here perhaps indicate wider issues of paternalism and attempts to control deaf populations. In particular, this links to widespread assertions within $d /$ Deaf communities that d/Deaf people are not disabled (see Lane 1999, 2002; Bathard 2012). Furthermore, Adam's attempts to help deaf people, whilst undoubtedly genuine, place him in a particular category within the d/Deaf community, which does not necessarily confer full inclusion and recognition as a culturally Deaf individual. Instead, positioning himself as someone who can 'help' may serve to categorise him as someone at the fringes of Deaf culture who can gate-keep between d/Deaf and hearing worlds. In this respect, Irene Leigh, a psychologist, states that "it can be stressful to figure out how and when to exhibit certain behaviors in order to maximize acceptability, how to manage situations when behaviour unacceptable within one culture is witnessed, and how to avoid the impression of dominance (e.g., spoken-language values) when acculturating to the nondominant (e.g., Deaf) culture" (2009: 50). On becoming involved with Deaf communities, the $\mathrm{Cl}$ users in this study were required to learn new cultural protocols in order to be accepted and to fit in, which involves adopting and thus displaying values, behaviours and attitudes that are typical of $d$ /Deaf people, and distinct from hearing people. Such uneasiness about the correct ways in which to interact on the edges of $d$ /Deaf and hearing worlds adds to $\mathrm{Cl}$ users' sense of liminality, as they become conscious of their inability to integrate smoothly into these worlds. On top of this, such difficulties in establishing a position within d/Deaf social worlds is reinforced by their primary involvement in hearing worlds until adolescent years, which can make their 'allegiances' unclear or suspect.

In terms of belonging to either $d /$ Deaf or hearing social groups, Isaac stated that "both groups consider me to belong to the other group." Therefore, his own identity and place in either world is complicated by outsider perceptions of which group he belongs in. Such 
views reflect Snow and Anderson's (1987) concept of 'social identities,' and what Sheridan (2008) refers to as 'overt identities,' which denote the identities assigned to us by others.

Isaac: [...] for the $d /$ Deaf side of things, they see me signing, if they see me only signing, they'll assume I'm d/Deaf. But they also see me talking, and I try not to make a point of it because it's culturally insensitive, but I will talk around deaf people, and they do pick up on it. They notice I'm using my mouth and having a conversation with someone who they know is hearing, and so from that side of things, they see me as a hearing person, or at least I have a capability to speech that, to them, places me into a hearing category of things. From the hearing side of things, they see me being able to sign and put me in the $d /$ Deaf category [laughs]. Or they see the cochlear implant, hearing aids, and think, 'oh he's deaf.' And then when they hear me speak, they're like, 'oh he's deaf but he can speak, then he's kind of a hearing person.' So, each group kind of, they use social markers and identifiers to be like, 'that makes him part of my group, that makes him part of their group.'

These perceptions have the potential to make participating and belonging in either $d /$ Deaf or hearing social groups more complex, with both groups, according to Isaac, not attributing him in-group status, leading to a sense of being socially betwixt and between.

\section{CONCLUSION}

Despite general positivity amongst the $\mathrm{Cl}$ users in this study of their ability to be involved in both $d /$ Deaf and hearing worlds, the narratives that I have drawn out in this chapter illustrate the position of liminality that they occupy between these two worlds. This chapter focused primarily on the $\mathrm{Cl}$ users' varied journeys into the d/Deaf world, consequent interactions with other $d / D$ eaf people, and how these experiences illustrate their position on the margins of this world. Such a positioning is reflective of their upbringing in the hearing world and their previous desires to be immersed in this world (perhaps in an attempt to be seen as 'normal'), as well as the effect that their Cls and their speech have on the ways in which d/Deaf people perceive them. Given that these participants are still young adults, they are still negotiating their trajectory between and within these two worlds, along 91 
with the multiple factors that have shaped their identities over time. However, in drawing out the $\mathrm{Cl}$ users' narratives of having 'the best of both worlds,' I show how they normalise and positively value a self that is multi-layered, and a social world that is complexly arranged. Their identity work therefore involves asserting the value of a flexible, adaptable self, the value of a hybrid identity, and the effects of others' views of them that sometimes challenge their own narratives as Deaf and hearing. 


\section{CHAPTER EIGHT: CONCLUSIONS}

Through exploring the multiple threads that affect the identities of this small sample of cochlear implant users in New Zealand, I have presented a nuanced view of their lives, their lived experiences, their $d /$ Deaf bodies, and their complex, fluid identities. In that these young individuals have forged, and are still forging, their identities in the face of multiple social influences from multiple spheres, this thesis explores their identity trajectories between, and within, d/Deaf and hearing worlds. Thus, I demonstrate that these cochlear implant users are in the process of navigating complex journeys of finding their 'place' in the world, and establishing coherent narratives about authentic selves (Giddens 1991). This thesis is not representative of the experiences of cochlear implant users in New Zealand, however, and presents only a small sample of this, and the d/Deaf, population. Whilst it would have been both interesting and useful to include voices of those who have had little or no success with $\mathrm{Cls}$ here, the scope of this study and the variability of such voices limited my ability to include them. Despite such limitations in my research, this thesis contributes to a number of gaps in the literature. Within medical anthropology, I contribute to the study of disability - a vastly under-researched area within the discipline - as well as to theorisations of the body, illness narratives, and the study of liminality. Furthermore, this thesis contributes to the literature on cochlear implants, providing an alternative ethnographic approach to the identity formation of these individuals, rather than a specific focus on language acquisition or the effectiveness of cochlear implants more generally, for example. Instead, my thesis represents the subtleties of the experiences of $\mathrm{Cl}$ users, exploring the multiplicity of factors that influence their formations of self-narratives and self-identities (Snow and Anderson 1987, Giddens 1991).

Despite my participants' success with $\mathrm{Cls}$, both $\mathrm{Cl}$ users and their parents are situated within medical and social paradigms that continue to read $d /$ Deaf bodies as abnormal and which tend to emphasise the importance of fitting into the mainstream, hearing world. Furthermore, such ideals accentuate that these $\mathrm{Cl}$ users should embody ideas of successful, independent individuals in contemporary New Zealand society. Chapter Two demonstrates that, right from when deafness is diagnosed, or when a $\mathrm{Cl}$ is chosen, deaf individuals and their families become embedded in systems which medicalise deafness and emphasise the 93 
importance of the 'normal,' hearing body, which is capable of using spoken language. Thus, the decisions surrounding implantation, and those that follow (primarily about communication and education), focus on the correction of an 'impairment.' Despite the attempts of some parents in this study to challenge these systems after implantation through attempts to maintain New Zealand Sign Language and to facilitate involvement in the $d /$ Deaf world, for example - the emphasis within mainstream medical and educational systems and the work required to 'make the most' of the $\mathrm{Cl}$ technology, made these efforts difficult to sustain. Both parents and $\mathrm{Cl}$ users in this study, however, focused, in their narratives on making good decisions, on the choices and opportunities that they believed having a $\mathrm{Cl}$ would elicit, and on the construction of autonomous empowered individuals, and they narratively foregrounded the goals of successful and authentic participation in d/Deaf and hearing worlds. However, in Chapter Five, I problematized such notions of "choice," asserting that choices can be heavily circumscribed by social norms, technological limits and cultural values and that individuals are never, in fact, entirely autonomous (Mol 2008). In this respect, the $\mathrm{Cl}$ users in this study did not have completely 'free' choice about their implants and whether to continue use later in life, as they remain embedded in spheres where expectations that they would maintain their hearing and speech subtly prevailed. Lastly, the decisions made about implants can feel irreversible, given the amount of time and money invested in them, and that they give users the ability to use spoken language, a fact which may always differentiate the $d /$ Deaf bodies of $\mathrm{Cl}$ users from other $d / D$ eaf bodies.

The nuanced experiences of cochlear implant users are drawn out in Chapter Four, in which the narratives of my participants demonstrate the everyday complications inherent in using this technology. Whilst Cls were generally referred to positively by all participants, it is important to note the varied effects of $\mathrm{Cls}$, and the limits of the technology. $\mathrm{Cl}$ users became acutely aware of the fragile, fallible nature of implants when they stopped working, or when they found themselves in situations where the implants were ineffective. Furthermore, $\mathrm{Cl}$ users emphasised the difficult nature of 'hearing' with a $\mathrm{Cl}$, especially in loud places or group situations. Moreover, my participants emphasised the need for increased awareness of both $d /$ Deafness and cochlear implants, and the importance of recognition that a $\mathrm{Cl}$ does not, in fact, make them 'hearing,' nor, therefore, an automatic member of wider hearing society. 
In the midst of navigating the medical and parental pressures associated with $d /$ Deafness and $\mathrm{Cls}$, as well as coming to terms with the embodied experiences of the technology, the $\mathrm{Cl}$ users in this study were exploring and discovering their own identity trajectories (Snow and Anderson 1987). In particular, the narratives of my participants emphasised the pull of the $d /$ Deaf community in their adolescent years, and the importance of connections with other $d /$ Deaf people, reflecting that involvement in the $d /$ Deaf world is central in the formation of their senses of an authentic self. Furthermore, these participants created narratives throughout interviews that highlighted the ways in which they have successfully incorporated social ties in the $d /$ Deaf world with their place in the hearing world. Yet their narratives were not completely coherent, as self-narratives never are (Riessman 2008). They also described how negotiating such worlds was a difficult process, when they were excluded from full membership in either, or when they felt a liminal sense of peripheral belonging. Their identity work thus involved presenting themselves as empowered, successful and actualised individuals, at the same time that they reflected on their limited agency, and the difficulties of living in between Deaf and hearing identities and social spheres.

\section{Recommendations From Participants: Good Practice for Cochlear} IMPLANTS AND THEIR USERS

Some of the participants in my study highlighted the importance of circulating realistic information about the technology, especially given the variability of outcomes. Olivia, a teacher of the deaf, voiced particular concerns on how the realities of $\mathrm{Cls}$ may be conveyed to parents of deaf children, stating, "there's not a predictable outcome, even if a kid is an ideal candidate the outcome doesn't necessarily match, [and] they're not really any better at predicting the outcome now than they were 30 years ago." Furthermore, Olivia noted concerns over medicalised approaches to deafness, particularly inherent in ideas of 'fixing' deafness, and thus how Cls are presented to parents as a straightforward, successful remedy, or cure. Sandra, Adam's mother, further emphasised that the varied outcomes of Cls, as well as the need for support after surgery, need to be conveyed to those making decisions. She noted, "it's not something that's just black and white. It's not just about the implant, it's about the family, the circumstance, the back-up, the support, the awareness." 
The $\mathrm{Cl}$ user participants in this study made clear recommendations on what they believe should be good practice for $d /$ Deaf children with cochlear implants, as well as what parents and medical professionals should be aware of when it comes to this technology. Whilst all of these participants were positive about their experiences with Cls, they were adamant that the technology has not made them 'hearing,' and that this needs to be conveyed to parents of deaf children in particular. Specifically, Jack, Hannah and Adam were resolute that children with implants should grow up using both spoken and signed languages. On this, Hannah stated, "once they're implanted, they [don't just] want [to], they need to learn sign language. [T]here's no argument, end of conversation [...]." Furthermore, Adam spoke of how, as a $\mathrm{Cl}$ user, he should be able to use both spoken and signed languages: "if I went to a hearing school I should be able to speak and communicate with everyone else, but I should be able to communicate with my links [the $d /$ Deaf world]. I shouldn't be told to stop doing one of these things [signed or spoken language], I should be encouraged to do both." Adam's recommendation here is powerful given that he was required to stop using sign language at school in order to improve his spoken language. Additionally, his narrative highlights the centrality of his d/Deafness, but also the importance of maintaining links and connection in the hearing world. The recommendations of these participants, then, do not discourage the use of implants, but rather encourage solutions to make their journeys between, and within, $d /$ Deaf and hearing worlds a little easier.

\section{CONCLUDING THOUGHTS}

I argue that the identities of the $\mathrm{Cl}$ users in this study are influenced by, and forged through, the multiple threads and social influences of both $d /$ Deaf and hearing worlds. Thus, this thesis tracks the identity journeys of these individuals between, and within, $d /$ Deaf and hearing spheres, and explores the liminal space that they occupy. I have demonstrated that multiple factors shape the identities and self-narratives of these $\mathrm{Cl}$ users over time medicalised notions of deafness, the expectations surrounding $\mathrm{Cl}$ technology, the hopes and expectations of parents for their children, the embodied experience of having a $\mathrm{Cl}$, the effects and limits of the technology, social norms of both $d /$ Deaf and hearing worlds, and individual identity trajectories (especially opportunities for involvement in $d /$ Deaf worlds). 
This thesis thus demonstrates that, in constructing reflexive narratives about their lives and identities, young deaf adults with Cls emphasise the effects of wider social and familial forces and influences on their lives, their own agency to choose and adjust to the technology, their flexibility and expertise in navigating diverse and sometimes incongruent social arenas, and their strength and determination in dealing with all the challenges; all of which help them to construct a meaningful and ultimately positive life story.

It is important to recognise and support cochlear implant users as they negotiate complex lives between $d /$ Deaf and hearing worlds. Furthermore, it is vital that their needs as $d /$ Deaf people are met within both medical and social spheres. Most importantly, the voices of cochlear implant users themselves should be brought to the fore and become central in the decisions made about them, and about good practice for individuals like them. My participants demonstrated rich reflexivity and awareness of their complex negotiations of $d /$ Deaf and hearing worlds, which can significantly contribute to discussions on $d /$ Deafness and cochlear implants. 


\section{BIBLIOGRAPHY}

Abu-Lughod, Lila.

1986 Veiled Sentiments: Honor and Poetry in a Bedouin Society. Berkeley: University of California Press.

Andersson, Yerker, and Susan Burch.

2010 Deaf and Disability Studies: A Conversation with Yerker Andersson. In Deaf and Disability Studies: Interdisciplinary Perspectives. Susan Burch and Alison Kafer, eds. Pp. 193-203. Washington, D.C.: Gallaudet University Press.

Andrews, Jean F., Irene W. Leigh and Mary T. Weiner.

2004 Deaf People: Evolving Perspectives from Psychology, Education, and Sociology. Boston: Pearson Education.

Antia, Shirin D., Kathryn H. Kreimeyer, and Susanne Reed.

2010 Supporting Students in General Education Classrooms. In The Oxford Handbook of Deaf Studies, Language, and Education, vol. 2. Marc Marschark and Patricia Elizabeth Spencer, eds. Pp. 72-92. New York: Oxford University Press.

Archbold, Sue M., Mark E. Lutman, Susan Gregory, Ciaran O'Neill, and Thomas P. Nikolopoulos.

2002 Parents and Their Deaf Child: Their Perceptions Three Years after Cochlear Implantation. Deafness \& Education International 4(1): 12-40.

Archbold, Sue, and Alexandra Wheeler.

2010 Cochlear Implants: Family and Young People's Perspectives. In The Oxford Handbook of Deaf Studies, Language, and Education, vol. 2. Marc Marschark and Patricia Elizabeth Spencer, eds. Pp. 226-240. New York: Oxford University Press.

Baker, Charlotte, and Dennis Cokely.

1980 American Sign Language: a teacher's resource text on grammar and culture. Maryland: T.J. Publishers Inc.

Bat-Chava, Yael.

2000 Diversity of Deaf Identities. American Annals of the Deaf 145(5): 420-428. 
Bathard, Hayley.

2012 "I'm not sick": The fraught interactions of d/Deafness and disability. Honours dissertation, Anthropology, Victoria University of Wellington.

Bathard, Hayley, Rosie Broad, Ruth Fitzgerald and Michael Legge.

2013 Conversations with 3 South Island families about the lived experience of heritable deafness, the meanings of disability and reproductive decision-making. Technical Report. Department of Archaeology and Anthropology, University of Otago, New Zealand.

Bauman, H-Dirksen and Joseph Murray.

2010 Deaf Studies in the $21^{\text {st }}$ Century: "Deaf-gain" and the Future of Human Diversity. In Oxford Handbook of Deaf Studies and Deaf Education, vol. 2. Marc Marschark and Patricia Elizabeth Spencer, eds. Pp. 210-225. New York: Oxford University Press.

Bell, Lara Joyce Milka.

2013 The Disrupted and Realigned Self: Exploring the Narratives of New Zealanders with Chronic Fatigue Syndrome/Myalgic Encephalomyelitis. M.A. thesis, Anthropology, Victoria University of Wellington.

Bernard, H. Russell.

2002 Research Methods in Anthropology: Qualitative and Quantitative Approaches. $3^{\text {rd }}$ edition. Walnut Creek: AltaMira Press.

Best, Alana, Corinna Howland and Julie Park.

2012 Auckland Deaf Families Study: "Troubling Choice" Summer Student Research Report. Department of Anthropology, University of Auckland, New Zealand.

Best, Alana, Corinna Howland, Jenny Snapp, and Julie Park.

2013 Eugenics and Utopia: Social Imaginaries of Technologies for Deafness. Sites: A Journal of Social Anthropology and Cultural Studies 10(2): 107-128.

Blume, Stuart S.

1997 The Rhetoric and Counter-Rhetoric of a "Bionic" Technology. Science, Technology, and Human Values 22(1): 31-56. 
Blume, Stuart.

2010 The Artificial Ear: Cochlear Implants and The Culture of Deafness. New Brunswick, New Jersey and London: Rutgers University Press.

Brewer, John D.

2000 Ethnography. Buckingham: Open University Press.

Brockmeier, Jens, and Donal Carbaugh.

2001 Introduction. In Narrative and Identity: Studies in Autobiography, Self and Culture. Jens Brockmeier and Donal Carbaugh, eds. Pp. 1-22. Amsterdam: John Benjamins Publishing Company.

Brueggemann, Brenda Jo.

2009 Deaf Subjects: Between Identities and Places. New York and London: New York University Press.

Bury, Mike.

2001 Illness Narratives: Fact or Fiction? Sociology of Health and Illness 23(3): 263285.

Byrne, Bridget.

2003 Reciting the Self: Narrative Representations of the Self in Qualitative Interviews. Feminist Theory 4(1): 29-49.

Callero, Peter L.

2003 The Sociology of the Self. Annual Review of Sociology 29(1): 115-133.

Charmaz, Kathy.

1990 "Discovering" Chronic Illness: Using Grounded Theory. Social Science \& Medicine 30(11): 1161-1172.

Christiansen, John B., and Irene W. Leigh.

2002 Cochlear Implants in Children: Ethics and Choices. Washington, DC: Gallaudet University Press.

Christiansen, John B., and Irene W. Leigh.

2004 Children With Cochlear Implants: Changing Parent and Deaf Community Perspectives. Archives of Otolaryngology - Head and Neck Surgery 130(5): 673-677. 
Christiansen, John B., and Irene W. Leigh.

2011 Cochlear Implants and Deaf Community Perceptions. In Cochlear Implants: Evolving Perspectives. Raylene Paludneviciene and Irene W. Leigh, eds. Pp. 39-55. Washington, DC: Gallaudet University Press.

Cochlear Implants in New Zealand.

What is a Cl? http://2ears2hear.kiwi.nz/resources/what-is-a-ci/.

Conrad, Peter.

1992 Medicalization and Social Control. Annual Review of Sociology 18(1): 209-232.

Conrad, Peter.

2005 The Shifting Engines of Medicalization. Journal of Health and Social Behavior 46(1): 3-14.

Corbin, Juliet, and Anselm Strauss.

1990 Grounded Theory Research: Procedures, Canons, and Evaluative Criteria. Qualitative Sociology 13(1): 3-21.

Corker, Mairian.

1998 Disability Discourse in a Postmodern World. In The Disability Reader: Social Science Perspectives. Tom Shakespeare, ed. Pp. 221-233. London and New York: Cassell.

Csordas, Thomas.

1984 Embodiment as a Paradigm for Anthropology. Ethos 18(5): 5-47.

DANZ.

Deaf Aotearoa New Zealand. http://www.deaf.org.nz.

Das, Veena, and Renu Addlakha.

2007 Disability and Domestic Citizenship: Voice, Gender, and the Making of the Subject. In Disability in Local and Global Worlds. Benedicte Ingstad and Susan Reynolds Whyte, eds. Pp. 128-148. California: University of California Press.

Davis, Lennard J.

1995 Enforcing Normalcy: Disability, Deafness, and the Body. New York: Verso. 
Davis, Lennard J.

2007 Deafness and the Riddle of Identity. The Chronicle of Higher Education 53(19).

Davis, Lennard J.

2013a Introduction: Normality, Power, and Culture. In The Disability Studies Reader. $4^{\text {th }}$ edition. Lennard J. Davis, ed. Pp. 1-14. New York and Oxon: Routledge.

Davis, Lennard J.

2013b The End of Identity Politics: On Disability as an Unstable Category. In The Disability Studies Reader. $4^{\text {th }}$ edition. Lennard J. Davis, ed. Pp. 263-277. New York and Oxon: Routledge.

Dent, Mike.

2006 Patient choice and medicine in health care. Public Management Review 8(3): 449-462.

Doe, Tanis.

2004 The Difficulty with Deafness Discourse and Disability Culture. The Review of Disability Studies 1(1): 34-41.

Dolnick, Edward.

1993 Deafness as Culture. The Atlantic Monthly 272(3): 37-53.

Edwards, R. A. R.

2005 Sound and Fury; Or, Much Ado About Nothing? Cochlear Implants in Historical Perspective. Journal of American History 92(3): 892-920.

FDA.

2010 What is a cochlear implant? U.S. Department of Health and Human Services. http://www.fda.gov/MedicalDevices/ProductsandMedicalProcedures/ImplantsandP rosthetics/Cochlearlmplants/ucm062823.htm.

Fitzgerald, Ruth Patricia, Hayley Bathard, Rosie Broad and Michael Legge.

2013 Reproduction, ethics and heritable deafness: three South Island families express their views. Sites: A Journal of Social Anthropology and Cultural Studies 10(2): 129-149. 
Foster, Susan, and Waithera Kinuthia.

2003 Deaf Persons of Asian American, Hispanic American, and African American Backgrounds: A Study of Intraindividual Diversity and Identity. Journal of Deaf Studies and Deaf Education 8(3): 271-290.

Friedner, Michele.

2010 Focus on Which (Deaf) Space? Identity and Belonging among Deaf Women in New Delhi, India. In Deaf and Disability Studies: Interdisciplinary Perspectives. Susan Burch and Alison Kafer, eds. Pp. 48-66. Washington, D.C.: Gallaudet University Press.

Gale, Elaine.

2011 Exploring Perspectives on Cochlear Implants and Language Acquisition Within the Deaf Community. Journal of Deaf Studies and Deaf Education 16(1): 121-139.

Garland-Thomson, Rosemarie.

2007 Shape Structures Story: Fresh and Feisty Stories About Disability. Narrative 15(1): 113-123.

Geertz, Clifford.

1973 The Interpretation of Cultures: Selected Essays. New York: Basic Books.

Giddens, Anthony.

1991 Modernity and Self-Identity: Self and Society in the Late Modern Age. Cambridge: Polity Press.

Glickman, Neil S.

1996 The Development of Culturally Deaf Identities. In Culturally Affirmative Psychotherapy with Deaf Persons. Neil S. Glickman and Michael A. Harvey, eds. Pp. 115-153. Mahwah, New Jersey: Lawrence Erlbaum Associates, Publishers.

Goffman, Erving.

1963 Stigma: Notes on the Management of Spoiled Identity. Englewood Cliffs: Prentice-Hall.

Gray, Caroline.

2009 Narratives of Disability and the Movement from Deficiency to Difference. Cultural Sociology 3(2): 317-332. 
Grosjean, Francois.

2010 Bilingualism, biculturalism, and deafness. International Journal of Bilingual Education and Bilingualism 13(2): 133-145.

Guillemin, Marilys, and Lynn Gillam.

2006 Attitudes to Genetic Testing for Deafness: The Importance of Informed Choice. Journal of Genetic Counseling 15(1): 51-59.

Hall, Stuart.

2000 Who needs 'identity'? In Identity: A Reader. Paul du Gay, Jessica Evans, and Peter Redman, eds. Pp. 15-30. London: Sage Publications.

Haualand, Hilde.

2007 The Two-Week Village: The Significance of Sacred Occasions for the Deaf Community. In Disability in Local and Global Worlds. Benedicte Ingstad and Susan Reynolds Whyte, eds. Pp. 33-55. California: University of California Press.

Holcomb, Thomas K.

1997 Development of Deaf Bicultural Identity. American Annals of the Deaf 142(2): 89-93.

Hyde, Merv, and Des Power.

2006 Some Ethical Dimensions of Cochlear Implantation for Deaf Children and Their Families. Journal of Deaf Studies and Deaf Education 11(1): $102-111$.

Hyde, Merv, Renee Punch, and Linda Komesaroff.

2010 Coming to a Decision About Cochlear Implantation: Parents Making Choices for Their Deaf Children. Journal of Deaf Studies and Deaf Education 15(2): 162-178.

Inhorn, Marcia C., and Emily A. Wentzell.

2012 Introduction: Medical Anthropology at the Intersections. In Medical Anthropology at the Intersections. Marcia C. Inhorn and Emily A. Wentzell, eds. Pp. 1-20. Durham and London: Duke University Press.

Ingstad, Benedicte, and Susan Reynolds Whyte.

2007 Introduction. In Disability in Local and Global Worlds. Benedicte Ingstad and Susan Reynolds Whyte, eds. Pp. 1-29. California: University of California Press. 
Jackson, Michael.

1998 Minima Ethnographica: Intersubjectivity and the Anthropological Project. Chicago and London: The University of Chicago Press.

Jaye, Chrystal, and Ruth Fitzgerald.

2012 The Embodied Liminalities of Occupational Overuse Syndrome. Medical Anthropology Quarterly 26(2): 201-220.

Karkazis, Katrina.

2008 Fixing Sex: Intersex, Medical Authority, and Lived Experience. Durham and London: Duke University Press.

Kasnitz, Devva, and Russell P. Shuttleworth.

2001 Introduction: Anthropology in Disability Studies. Disability Studies Quarterly 21(3): 2-17.

Kelly, Angela.

2008 Living loss: an exploration of the internal space of liminality. Mortality: Promoting the interdisciplinary study of death and dying 13(4): 335-350.

Kent, B.A.

2003 Identity Issues for Hard-of-Hearing Adolescents Aged 11, 13, and 15 in Mainstream Settings. Journal of Deaf Studies and Deaf Education 8(3): 315-324.

Kermit, Patrick.

2009 Deaf or deaf? Questioning alleged antinomies in the bioethical discourses on cochlear implantation and suggesting an alternative approach to $d /$ Deafness. Scandinavian Journal of Disability Research 11(2):159-174.

Kleinman, Arthur.

1988 The Illness Narratives: Suffering, Healing and the Human Condition. United States of America: Basic Books.

Kochhar, Amit, Michael S. Hildebrand, and Richard J.H. Smith.

2007 Clinical Aspects of Hereditary Hearing Loss. Genetics in Medicine 9(7): 393408. 
Ladd, Paddy.

2006 What Is Deafhood and Why Is It Important? In The Deaf way II reader: perspectives from the second international conference on deaf culture. Harvey Goodstein, ed. Pp. 245-250. Washington, D.C.: Gallaudet University Press.

Laing, Patricia.

2006 Migrating to a Deaf World: A Model for Understanding the Experiences of Hearing Parents of Deaf Children. Sites: a Journal of Social Anthropology and Cultural Studies 3(1): 75-99.

Landsman, Gail Heidi.

2009 Reconstructing Motherhood and Disability in the Age of "Perfect" Babies. New York and Oxon: Routledge.

Lane, Harlan, Robert Hoffmeister and Ben Bahan.

1996 A Journey into the Deaf-World. San Diego, California: Dawn Sign Press.

Lane, Harlan, and Michael Grodin.

1997 Ethical Issues in Cochlear Implant Surgery: An Exploration into Disease, Disability, and the Best Interests of the Child. Kennedy Institute of Ethics Journal 7(3): 231-251.

Lane, Harlan.

1999 [1992] The Mask of Benevolence: Disabling the Deaf Community. San Diego: DawnSignPress.

Lane, Harlan.

2002 Do Deaf People Have a Disability? Sign Language Studies 2(4): 356-379.

Leigh, Irene W., Alan L. Marcus, Patricia K. Dobosh, and Thomas E. Allen.

1998 Deaf/Hearing Cultural Identity Paradigms: Modification of the Deaf Identity Development Scale. Journal of Deaf Studies and Deaf Education 3(4): 329-338.

Leigh, Irene W.

2008 Who Am I? Deaf Identity Issues. In Signs and Voices: Deaf Culture, Identity, Language, and Arts. Kristin A. Lindgren, Doreen DeLuca, and Donna Jo Napoli, eds. Pp. 21-29. Washington, DC: Gallaudet University Press. 
Leigh, Irene W.

2009 A Lens on Deaf Identities. Oxford, New York: Oxford University Press.

Leigh, Irene W., Deborah Maxwell-McCaw, Yael Bat-Chava, and John B. Christiansen.

2009 Correlates of Psychosocial Adjustment in Deaf Adolescents With and Without Cochlear Implants: A Preliminary Investigation. Journal of Deaf Studies and Deaf Education 14(2): 244-259.

Leigh, Irene W. and Deborah Maxwell-McCaw.

2011 Cochlear Implants: Implications for Deaf Identities. In Cochlear Implants: Evolving Perspectives. Raylene Paludneviciene and Irene W. Leigh, eds. Pp. 95-110. Washington, DC: Gallaudet University Press.

Little, Miles, Christopher FC Jordens, Kim Paul, Kathleen Montgomery, and Bertil Philipson. 1998 Liminality: A Major Category of the Experience of Cancer Illness. Social Science \& Medicine 47(10): 1485-1494.

Lock, Margaret.

1993 Cultivating the Body: Anthropology and Epistemologies of Bodily Practice and Knowledge. Annual Review of Anthropology 22(1): 133-155.

Lock, Margaret.

2001 The Tempering of Medical Anthropology: Troubling Natural Categories. Medical Anthropology Quarterly 15(4): 478-492.

Lupton, Deborah.

2012 Medicine as Culture: Illness, Disease and the Body. $3^{\text {rd }}$ edition. Croydon, UK: Sage Publications.

Marschark, Marc, and Patricia Elizabeth Spencer.

2006 Spoken Language Development of Deaf and Hard-of-Hearing Children: Historical and Theoretical Perspectives. In Advances in the Spoken Language Development of Deaf and Hard-of-Hearing Children. Patricia Elizabeth Spencer and Marc Marschark, eds. Pp. 3-21. New York: Oxford University Press.

Martland, T.R.

1975 On "The Limits of My Language Mean the Limits of My World". The Review of Metaphysics 29(1): 19-26. 
Marx, Karl.

1887 Capital: A Critique of Political Economy, Volume 1. Samuel Moore and Edward Aveling, trans. Moscow: Progress Publishers.

Mattingly, Cheryl, and Linda C. Garro.

2000 Narrative as Construct and Construction. In Narrative and the Cultural Construction of Illness and Healing. Cheryl Mattingly and Linda C. Garro, eds. Pp. 149. Berkeley: University of California Press.

Maxwell-McCaw, Deborah L., Irene W. Leigh, and Alan L. Marcus.

2000 Social Identity in Deaf Culture: A Comparison of Ideologies. JADARAROCHESTER NY 33(3): 14-28.

Mcllroy, Guy, and Claudine Storbeck.

2011 Development of Deaf Identity: An Ethnographic Study. Journal of Deaf Studies and Deaf Education 16(4): 494-511.

McKee, Rachel Locker.

2006 Connecting Hearing Parents with the Deaf World. SITES: New Series 3(1): 143167.

McKee, Rachel Locker.

2008 The Construction of Deaf Children as Marginal Bilinguals in the Mainstream. International Journal of Bilingual Education and Bilingualism 11(5): 519-540.

Messinger, Seth D.

2010 Getting Past the Accident: Explosive Devices, Limb Loss, and Refashioning a Life in a Military Centre. Medical Anthropology Quarterly 24(3): 281-303.

Mishler, Elliot G.

2004 Historians of the Self: Restorying Lives, Revising Identities. Research in Human Development 1(1-2): 101-121.

Mitchell, Ross E., and Michael A. Karchmer.

2004 Chasing the Mythical Ten Percent: Parental Hearing Status of Deaf and Hard of Hearing Students in the United States. Sign Language Studies 4(2): 138-163. 
Mitchiner, Julie and Marilyn Sass-Lehrer.

2011 My Child Can Have More Choices: Reflections of Deaf Mothers on Cochlear Implants for Their Children. In Cochlear Implants: Evolving Perspectives. Raylene Paludneviciene and Irene W. Leigh, eds. Pp. 71-94. Washington, DC: Gallaudet University Press.

Mol, Annemarie.

2008 [2006] The Logic of Care: Health and the Problem of Patient Choice. London and New York: Routledge.

Moore, Michele, Sarah Beazley and June Maelzer.

1998 Researching Disability Issues. Buckingham, Philadelphia: Open University Press.

Most, Tova, Amatzia Wiesel and Tamar Blitzer.

2007 Identity and Attitudes towards Cochlear Implant Among Deaf and Hard of Hearing Adolescents. Deafness and Education International 9(2): 68-82.

Murphy, Robert F., Jessica Scheer, Yolanda Murphy, and Richard Mack.

1988 Physical Disability and Social Liminality: A Study in the Rituals of Adversity. Social Science \& Medicine 26(2): 235-242.

Murphy, Robert.

1990 [1987] The Body Silent. New York and London: W.W. Norton.

NFD.

2007 About us. http://www.nfd.org.nz/6/About-Us.

New Zealand Ministry of Education

2014 Services and funding for school students with high special education needs. http://www.minedu.govt.nz/Parents/AllAges/SupportForChildrenWithSpecialNeeds /ServicesAndFundingForStudentsWithHighNeeds.aspx.

NZ Audiological Society.

2011 Tell me about cochlear implants. http://www.audiology.org.nz/aboutcochlear-implants.aspx. 
O’Byrne, Ryan.

2012 "So we thought not to lose our background completely": Agency and belonging among South Sudanese Acholi in New Zealand. M.A. thesis, Anthropology, Victoria University of Wellington.

O’Neil, Andrea.

2012 Benefits of implants come through loud and clear. Kapi-Mana News. http://www.stuff.co.nz/dominion-post/news/local-papers/kapi-mananews/7604691/Benefits-of-implants-come-through-loud-and-clear.

Ohna, Stein Erik.

2004 Deaf in my own way: Identity, learning and narratives. Deafness and Education International 6(1): 20-38.

Oliver, Michael.

2009 Understanding Disability: From Theory to Practice. $2^{\text {nd }}$ edition. Basingstoke, New York: Palgrave Macmillan.

Padden, Carol, and Tom Humphries.

1988 Deaf in America: Voices from a Culture. United States of America: Harvard University Press.

Padden, Carol, and Tom Humphries.

2005 Inside Deaf Culture. United States of America: Harvard University Press.

Paludneviciene, Raylene, and Raychelle L. Harris.

2011 Impact of Cochlear Implants on the Deaf Community. In Cochlear Implants: Evolving Perspectives. Raylene Paludneviciene and Irene W. Leigh, eds. Pp. 3-19. Washington, DC: Gallaudet University Press.

Penman, Maryke.

2012 Rhianna's world opens up. North Shore Times. http://www.stuff.co.nz/auckland/local-news/north-shore-times/7606621/Rhiannasworld-opens-up.

Preisler, Gunilla, Anna-Lena Tvingstedt, and Margareta Ahlstrom.

2005 Interviews with Deaf Children About Their Experiences Using Cochlear Implants. American Annals of the Deaf 150(3): 260-267. 
Rapp, Rayna.

1999 Testing Women, Testing the Fetus: The Social Impact of Amniocentesis in America. New York and London: Routledge.

Rapp, Rayna.

2001 Gender, Body, Biomedicine: How Some Feminist Concerns Dragged Reproduction to the Center of Social Theory. Medical Anthropology Quarterly 15(4): 466-477.

Rapp, Rayna and Faye Ginsburg.

2012 Anthropology and the Study of Disability Worlds. In Medical Anthropology at the Intersections. Marcia C. Inhorn and Emily A. Wentzell, eds. Pp. 163-182. Durham and London: Duke University Press.

Riessman, Catherine Kohler.

1990 Strategic Uses of Narrative in the Presentation of Self and Illness: A Research Note. Social Science \& Medicine 30(11): 1195-1200.

Riessman, Catherine Kohler.

2008 Narrative Methods for the Human Sciences. Thousand Oaks: Sage Publications.

Sach, Tracey H., and David K. Whynes.

2005 Paediatric cochlear implantation: the views of parents. International Journal of Audiology 44(7): 400-407.

Sawicki, Nina.

2008 Listening through deaf ears: parental experiences of the wired world. Master of General Practice thesis, University of Otago, New Zealand.

Senghas, Richard J., and Leila Monaghan.

2002 Signs of their Times: Deaf Communities and the Culture of Language. Annual Review of Anthropology 31(1): 69-97.

Shadwell, Talia.

2012 World of sound through implants. Manawatu Standard. http://www.stuff.co.nz/manawatu-standard/features/7607280/World-of-soundthrough-implants. 
Shakespeare, Tom and Nick Watson.

2001 Making the Difference: Disability, Politics, and Recognition. In Handbook of Disability Studies. Gary L. Albrecht, Katherine D. Seelman and Michael Bury, eds. Pp. 546-564. United States of America: Sage Publications.

Shakespeare, Tom.

2005 Disability Studies Today and Tomorrow. Sociology of Health \& Illness 27(1): 138-148.

Shakespeare, Tom.

2006 Disability Rights and Wrongs. America and Canada: Routledge.

Shakespeare, Tom.

2013 The Social Model of Disability. In The Disability Studies Reader. $4^{\text {th }}$ edition. Lennard J. Davis, ed. Pp. 214-221. New York and Oxon: Routledge.

Sheridan, Martha A.

2008 Deaf Adolescents: Inner Lives and Lifeworld Development. Washington, D.C.: Gallaudet University Press.

Shuttleworth, Russell, and Devva Kasnitz.

2004 Stigma, Community, Ethnography: Joan Ablon's Contribution to the Anthropology of Impairment-Disability. Medical Anthropology Quarterly 18(2): 139161.

Skelton, Tracey, and Gill Valentine.

2003 'It feels like being Deaf is normal': an exploration into the complexities of defining D/deafness and young D/deaf people's identities. The Canadian Geographer 47(4): 451-466.

Smiler, Kirsten, and Rachel Locker McKee.

2006 Perceptions of Maori Deaf Identity in New Zealand. Journal of Deaf Studies and Deaf Education 12(1): 93-111.

Smith, Eileen.

2003 "Deaf Ways": Literacy Teaching Strategies of Deaf Teachers in New Zealand. M.A. thesis, Applied Linguistics, Victoria University of Wellington. 
Smith, Keri E. Iyall.

2008 Hybrid Identities: Theoretical Examinations. In Hybrid Identities: Theoretical and Empirical Examinations. Keri E. Iyall Smith and Patricia Leavy, eds. Pp. 3-11. Leiden: Brill.

Snapp, Jennifer Marie.

2012 We Wanted Her to Hear: Hearing Parents, Deaf Children and Cochlear Implants. M.A. thesis, Anthropology, University of Auckland.

Snow, David A., and Leon Anderson.

1987 Identity Work among the Homeless: The Verbal Construction and Avowal of Personal Identities. American Journal of Sociology 92(6): 1336-1371.

Sointu, Eeva.

2005 The rise of an ideal: tracing changing discourses of wellbeing. The Sociological Review 53(2): 255-274.

Sparrow, Robert.

2005 Defending Deaf Culture: The Case of Cochlear Implants. Journal of Political Philosophy 13(2): 135-152.

Steven, Dionne.

2013 Narratives of Incorporation: An Anthropological Analysis of Same-Sex Civil Unions in New Zealand. Ph.D. thesis, Anthropology, Victoria University of Wellington.

Swanwick, Ruth, and Isabel Tsverik.

2007 The Role of Sign Language for Deaf Children with Cochlear Implants: Good Practice in Sign Bilingual Settings. Deafness \& Education International 9(4): 214-231.

Tatum, Beverly Daniel.

1997 Why Are All the Black Kids Sitting Together in the Cafeteria? And Other Conversations About Race. United States of America: Basic Books.

Taylor, Cliff.

2011 Making sense in a hearing world. The New Zealand Herald. http://www.nzherald.co.nz/humanscience/news/article.cfm?c_id=314\&objectid $=10773878$. 
Thomas, Carole.

2007 Sociologies of Disability and Illness: Contested Ideas in Disability Studies and Medical Sociology. Basingstoke and New York: Palgrave Macmillan.

Torrie, Bronwyn.

2012 Cochlear implant opens new world for Jireh. Fairfax NZ News. http://www.stuff.co.nz/national/health/7507412/Cochlear-implant-opens-newworld-for-Jireh.

Trnka, Susanna, and Laura McLaughlan.

2012 Becoming "Half a Doctor": Parent-Experts and the Normalisation of Childhood Asthma in Aotearoa/New Zealand. Sites: A Journal of Social Anthropology and Cultural Studies 9(2): 3-22.

Trundle, Catherine.

2011 Biopolitical endpoints: Diagnosing a deserving British nuclear test veteran. Social Science and Medicine 73(6): 882-888.

Turner, Victor W.

1969 The Ritual Process: Structure and Anti-Structure. London: Routledge and Kegan Paul.

Turner, Victor.

1985 Liminality, Kabbalah, and the Media. Religion 15(3): 205-217.

Turner, Victor.

1987 Betwixt and Between: The Liminal Period in Rites of Passage. In Betwixt and Between: Patterns of Masculine and Feminine Initiation. Louise Carus Madhi, Steven Foster, and Meredith Little, eds. Pp. 3-19. La Salle: Open Court.

Turner, Victor.

1992 Blazing the Trail: Way Marks in the Exploration of Symbols. Tucson: The University of Arizona Press.

Valentine, Gill, and Tracey Skelton.

2007 Re-defining 'norms': D/deaf young people's transitions to independence. The Sociological Review 55(1): 104-123. 
van Asch Deaf Education Centre.

2008 van Asch Deaf Education Centre, Christchurch, New Zealand. http://www.vanasch.school.nz.

van Gennep, Arnold.

1960 The Rites of Passage. Monika B. Vizedom and Gabrielle L. Caffee, trans. London: Routledge and Kegan Paul.

Wald, Rebecca L., and John F. Knutson.

2000 Deaf cultural identity of adolescents with and without cochlear implants. The Annals of Otology, Rhinology and Laryngology 109(12): 87-89.

Watson, Nick.

2002 Well, I Know This Is Going to Sound Very Strange to You, but I Don't See Myself as a Disabled Person: Identity and Disability. Disability \& Society 17(5): 509527.

Wheeler, Alexandra, Sue Archbold, Susan Gregory, and Amy Skipp.

2007 Cochlear Implants: The Young People's Perspective. Journal of Deaf Studies and Deaf Education 12(3): 303-316.

Zaidman-Zait.

2007 Parenting a Child With a Cochlear Implant: A Critical Incident Study. Journal of Deaf Studies and Deaf Education 12(2): 221-241.

Zola, Irving Kenneth.

1982 Missing Pieces: A Chronicle of Living With a Disability. Philadelphia: Temple University Press. 
